\title{
Second-generation dual FXR/sEH modulators with optimized pharmacokinetics
}

Moritz Helmstädter ${ }^{1}$, Astrid Kaiser ${ }^{1}$, Steffen Brunst ${ }^{1}$, Jurema Schmidt ${ }^{1}$, Riccardo Ronchetti ${ }^{1}$, Lilia Weizel $^{1}$, Ewgenij Proschak ${ }^{1}$, Daniel Merk ${ }^{1 *}$

${ }^{1}$ Goethe University Frankfurt, Institute of Pharmaceutical Chemistry, 60438 Frankfurt, Germany

*merk@pharmchem.uni-frankfurt.de

Table of Contents

Chemistry

General

General procedures

Synthesis and analytical characterization of 4-29 and precursors 


\section{Chemistry}

$\underline{\text { General }}$

All chemicals and solvents were of reagent grade and used without further purification unless otherwise specified. All reactions were conducted in oven-dried glassware under argon atmosphere and in absolute solvents. NMR spectra were recorded on a Bruker AV 500, Bruker AV 400, Bruker AV 300 or a Bruker am250xp spectrometer (Bruker Corporation, Billerica, MA, USA). Chemical shifts $(\delta)$ are reported in ppm relative to tetramethylsilane (TMS) as reference. Multiplicity is reported: $\mathrm{s}$, singlet; $\mathrm{d}$, doublet; dd, doublet of doublets; t, triplet; q, quartett, qui, quintet; spt, septet; m, multiplet. Approximate coupling constants $(J)$ are given in hertz $(\mathrm{Hz})$. Mass spectra were obtained on a VG Platform II (Thermo Fischer Scientific, Inc., Waltham, MA, USA) using electrospray ionization (ESI). High-resolution mass spectra were recorded on a MALDI LTQ ORBITRAP XL instrument (Thermo Fisher Scientific). Melting points were determined on a Büchi M-560 (Büchi Labortechnik, Flawil, Switzerland). Compounds were purified by preparative HPLC using a Shimadzu preparative LC-20A Prominence

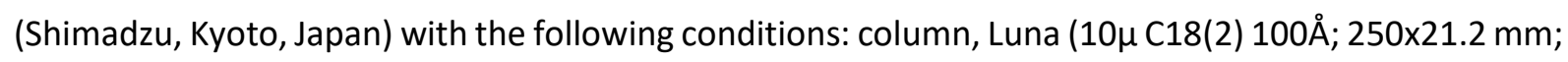
Phenomenex, Torrance, CA, U.S.A.); mobile phase, isocratic $50: 50$ acetonitrile $/ \mathrm{H}_{2} \mathrm{O}+0.1$ formic acid for $30 \mathrm{~min}$ at a flow rate of $21 \mathrm{~mL} / \mathrm{min}$ and UV-detection at $245 \mathrm{~nm}$ and $280 \mathrm{~nm}$. Compound purity was analyzed on a Waters 600 Controller HPLC (Waters, Milford, MA, U.S.A.) using a Waters 2487 Dual Absorbance Detector and a Waters 717 plus Autosampler or a VWR Chromaster (VWR, Radnor, PA, U.S.A.) with a 5160 pump system, using a DAD 5430 and 5260 Autosampler both equipped with a MultoHigh100 RP18-5 250x4 mm column (CS-Chromatographie Service GmbH, Langerwehe, Germany) using a gradient $\left(\mathrm{H}_{2} \mathrm{O}+0.1 \%\right.$ formic acid/MeOH 80:20 isocratic for 5 min to $\mathrm{MeOH}$ after additional $45 \mathrm{~min}$ and $\mathrm{MeOH}$ for additional $10 \mathrm{~min}$ ) at a flow rate of $1 \mathrm{~mL} / \mathrm{min}$ or a gradient $\left(\mathrm{H}_{2} \mathrm{O}+0.1 \%\right.$ formic acid/MeOH 60:40 isocratic for $5 \mathrm{~min}$ to $\mathrm{MeOH}$ after additional $25 \mathrm{~min}$ and $\mathrm{MeOH}$ for additional $10 \mathrm{~min}$ ) at a flow rate of $1 \mathrm{~mL} / \mathrm{min}$ with UV-detection at $245 \mathrm{~nm}$ and $280 \mathrm{~nm}$. All compounds used for biological testing had a purity $\geq 95 \%$.

\section{General procedures}

General procedure $\mathbf{A}$ for the synthesis of benzamides (52-75)

The respective benzoic acid derivative (33-51, 1.00 eq), the respective benzyl amine (30-32, 1.30-3.00 eq) and 4-(dimethylamino)pyridine (4-DMAP, 0.01-1.00 eq) were dissolved in a mixture of $\mathrm{CHCl}_{3}$ and DMF (6-23 ml/mmol; 1:1). After cooling the reaction mixture to $0^{\circ} \mathrm{C}$, EDC. $\mathrm{HCl}(1.00-3.00 \mathrm{eq})$ was added and the mixture was stirred at $0^{\circ} \mathrm{C}$ for 30 minutes and at $50^{\circ} \mathrm{C}$ for $16 \mathrm{~h}$. Then, aqueous hydrochloric acid $(10 \%, 15-30 \mathrm{~mL})$ was added and the phases were separated. The aqueous layer was brought to $\mathrm{pH}$ 12 using $\mathrm{Na}_{2} \mathrm{CO}_{3}$ and extracted EtOAc $(3 \times 10-30 \mathrm{~mL})$. The combined organic layers were dried over $\mathrm{Na}_{2} \mathrm{SO}_{4}$ and the solvent was removed in vacuum. Further purification was performed by column chromatography.

General procedure B for the synthesis of sulfonamides $(\mathbf{4 - 2 4 , 2 6 - 2 9 )}$

The respective aniline (52-72, 1.00 eq) was dissolved in a mixture of THF and pyridine $(1-30 \mathrm{ml} / \mathrm{mmol}$; 10:1). The respective sulfonyl chloride (76-80, 1-10 eq) was added dropwise. The reaction mixture was 
stirred under reflux for 2-16 hours. Then, aqueous hydrochloric acid (10\%, 15-30 mL) was added and the phases were separated. The aqueous layer was then extracted with EtOAc $(3 \times 10-30 \mathrm{~mL})$. The combined organic layers were dried over $\mathrm{Na}_{2} \mathrm{SO}_{4}$ and the solvent was removed in vacuum. Further purification was performed by column chromatography.

Synthesis and analytical characterization of 4-29 and precursors

4-(tert-Butyl)-N-(2-chloro-4-(ethylsulfonamido)benzyl)benzamide (4): Preparation according to general procedure B using $\mathrm{N}$-(4-amino-2-chlorobenzyl)-4-(tert-butyl)benzamide (52, $0.160 \mathrm{~g}, 0.510$ $\mathrm{mmol}, 1.00 \mathrm{eq})$ and ethanesulfonyl chloride $(74,0.656 \mathrm{~g}, 5.10 \mathrm{mmol}, 10.0 \mathrm{eq})$. The crude product was purified by column chromatography using $n$-hexane/EtOAc (1:1) as mobile phase to obtain 4 as a colorless solid (0.125 g, 60\%). M.p.: $198.6{ }^{\circ} \mathrm{C} .{ }^{1} \mathrm{H}$ NMR $\left(500 \mathrm{MHz}\right.$, DMSO- $\left.d_{6}\right) \delta=9.98(\mathrm{~s}, 1 \mathrm{H}), 8.93(\mathrm{t}, J=$ $5.8 \mathrm{~Hz}, 1 \mathrm{H}), 7.84(\mathrm{~d}, J=8.4 \mathrm{~Hz}, 2 \mathrm{H}), 7.49(\mathrm{~d}, J=8.4 \mathrm{~Hz}, 2 \mathrm{H}), 7.29(\mathrm{~d}, J=8.4 \mathrm{~Hz}, 1 \mathrm{H}), 7.27(\mathrm{~d}, J=2.1 \mathrm{~Hz}$, $1 \mathrm{H}), 7.15$ (dd, $J=8.4,2.1 \mathrm{~Hz}, 1 \mathrm{H}$ ), $4.47(\mathrm{~d}, J=5.7 \mathrm{~Hz}, 2 \mathrm{H}), 3.11(\mathrm{q}, J=7.3 \mathrm{~Hz}, 2 \mathrm{H}), 1.30(\mathrm{~s}, 9 \mathrm{H}), 1.18(\mathrm{t}, J$ $=3.6 \mathrm{~Hz}, 3 \mathrm{H}) .{ }^{13} \mathrm{C}$ NMR $\left(126 \mathrm{MHz}\right.$, DMSO- $\left.d_{6}\right) \delta=166.27,154.20,138.42,132.34,131.51,131.34,129.57$, $127.19,125.13,119.35,117.89,45.29,39.52,34.65,30.96,8.02$. HMRS (MALDI): $\mathrm{m} / \mathrm{z}$ calculated 409.13472 for $\mathrm{C}_{20} \mathrm{H}_{26} \mathrm{ClN}_{2} \mathrm{O}_{3} \mathrm{~S}$, found $409.13444\left([\mathrm{M}+\mathrm{H}]^{+}\right)$.

4-(tert-Butyl)-N-(2-chloro-4-(isopropylsulfonamido)benzyl)benzamide (5): Preparation according to general procedure B using $\mathrm{N}$-(4-amino-2-chlorobenzyl)-4-(tert-butyl)benzamide (52, $0.154 \mathrm{~g}, 0.490$ $\mathrm{mmol}, 1.00 \mathrm{eq})$ and isopropylsulfonyl chloride $(75,0.697 \mathrm{~g}, 4.89 \mathrm{mmol}, 10.0 \mathrm{eq})$. The crude product was purified by column chromatography using n-hexane/EtOAc (2:1) as mobile phase to obtain $\mathbf{5}$ as a yellow solid $(0.041 \mathrm{~g}, 20 \%)$. M.p.: $221.9{ }^{\circ} \mathrm{C} .{ }^{1} \mathrm{H}$ NMR $\left(500 \mathrm{MHz}\right.$, DMSO- $\left.d_{6}\right) \delta=9.95(\mathrm{~s}, 1 \mathrm{H}), 8.92(\mathrm{t}, J=$ $5.7 \mathrm{~Hz}, 1 \mathrm{H}), 7.84(\mathrm{~d}, J=8.4 \mathrm{~Hz}, 2 \mathrm{H}), 7.49(\mathrm{~d}, J=8.4 \mathrm{~Hz}, 2 \mathrm{H}), 7.36-7.24(\mathrm{~m}, 2 \mathrm{H}), 7.17(\mathrm{dd}, J=8.5,2.1 \mathrm{~Hz}$, $1 \mathrm{H}), 4.46(\mathrm{~d}, J=5.7 \mathrm{~Hz}, 2 \mathrm{H}), 3.28-3.20(\mathrm{~m}, 1 \mathrm{H}), 1.30(\mathrm{~s}, 9 \mathrm{H}), 1.23(\mathrm{~d}, J=6.8 \mathrm{~Hz}, 6 \mathrm{H}) \cdot{ }^{13} \mathrm{C} \mathrm{NMR}(126 \mathrm{MHz}$, DMSO- $\left.d_{6}\right) \delta=166.28,154.21,138.65,132.33,131.37,131.34,129.56,127.20,125.13,119.19,117.78$, 51.59, 40.56, 34.65, 30.96, 16.10. HMRS (MALDI): $\mathrm{m} / \mathrm{z}$ calculated 423.15037 for $\mathrm{C}_{21} \mathrm{H}_{28} \mathrm{CIN}_{2} \mathrm{O}_{3} \mathrm{~S}$, found $423.14967\left([\mathrm{M}+\mathrm{H}]^{+}\right)$.

4-(tert-Butyl)-N-(2-chloro-4-(cyclopropylsulfonamido)benzyl)benzamide (6): Preparation according to general procedure B using $\mathrm{N}$-(4-amino-2-chlorobenzyl)-4-(tert-butyl)benzamide (52, $0.150 \mathrm{~g}, 0.473$ $\mathrm{mmol}, 1.00 \mathrm{eq})$ and cyclopropanesulfonyl chloride $(76,0.333 \mathrm{~g}, 2.37 \mathrm{mmol}, 10.0 \mathrm{eq})$. The crude product was purified by column chromatography using $n$-hexane/EtOAc (3:1) as mobile phase to obtain $\mathbf{6}$ as a yellow solid (0.034 g, 17\%). M.p.: $213.3{ }^{\circ} \mathrm{C} .{ }^{1} \mathrm{H}$ NMR $\left(500 \mathrm{MHz}\right.$, Methanol- $\left.d_{4}\right) \delta=7.82-7.78(\mathrm{~m}, 2 \mathrm{H})$, $7.54-7.50(\mathrm{~m}, 2 \mathrm{H}), 7.37-7.33(\mathrm{~m}, 2 \mathrm{H}), 7.19(\mathrm{dd}, J=8.4,2.2 \mathrm{~Hz}, 1 \mathrm{H}), 4.62(\mathrm{~s}, 2 \mathrm{H}), 2.56(\mathrm{~m}, 1 \mathrm{H}), 1.35-$ $134(\mathrm{~m}, 9 \mathrm{H}), 1.07-1.02(\mathrm{~m}, 2 \mathrm{H}), 0.98-0.94(\mathrm{~m}, 2 \mathrm{H}) .{ }^{13} \mathrm{C}$ NMR $\left(126 \mathrm{MHz}\right.$, Methanol- $\left.d_{4}\right) \delta=170.30$, $156.55,139.88,134.68,133.11,132.46,130.80,128.29,126.56,122.53,120.45,42.05,35.80,31.55$, 30.56, 5.76. HRMS (MALDI): $\mathrm{m} / z$ calculated 421.13472 for $\mathrm{C}_{21} \mathrm{H}_{25} \mathrm{ClN}_{2} \mathrm{O}_{3} \mathrm{~S}$, found $421.13476\left([\mathrm{M}+\mathrm{H}]^{+}\right)$.

4-(tert-Butyl)-N-(2-chloro-4-(trifluoromethylsulfonamido)benzyl)benzamide $\quad$ (7): $\quad \mathrm{N}$-(4-Amino-2chlorobenzyl)-4-(tert-butyl)benzamide $(\mathbf{5 2}, 0.300 \mathrm{~g}, 0.987 \mathrm{mmol}, 1.00 \mathrm{eq})$ was dissolved in a mixture of THF ( $5 \mathrm{~mL}$ ) and pyridine $(5 \mathrm{~mL})$. Then trifluoromethanesulfonyl chloride $(77,0.239 \mathrm{~g}, 1.42 \mathrm{mmol}$, $1.50 \mathrm{eq}$ ) was added dropwise at $0^{\circ} \mathrm{C}$. The reaction mixture was stirred at $0^{\circ} \mathrm{C}$ for 8 hours. Then aqueous hydrochloric acid $(10 \%, 15 \mathrm{~mL})$ was added and the phases were separated. The aqueous layer was extracted with EtOAc $(3 \times 30 \mathrm{~mL})$. The combined organic layers were dried over $\mathrm{Na}_{2} \mathrm{SO}_{4}$ and the solvent 
was removed in vacuum. Further purification was performed by column chromatography using $n$ hexane/EtOAc (2:1) as mobile phase to obtain 7 as a yellow solid $(0.065 \mathrm{~g}, 15 \%)$. M.p.: $153.7^{\circ} \mathrm{C} .{ }^{1} \mathrm{H}$ $\operatorname{NMR}(500 \mathrm{MHz}$, Chloroform-d) $\delta=7.72-7.68(\mathrm{~m}, 2 \mathrm{H}), 7.44-7.41(\mathrm{~m}, 2 \mathrm{H}), 7.22(\mathrm{~d}, J=8.2 \mathrm{~Hz}, 1 \mathrm{H}), 6.70$ $(\mathrm{d}, J=2.4 \mathrm{~Hz}, 1 \mathrm{H}), 6.53(\mathrm{dd}, J=8.2,2.4 \mathrm{~Hz}, 1 \mathrm{H}), 4.59(\mathrm{~d}, J=5.8 \mathrm{~Hz}, 2 \mathrm{H}), 1.32(\mathrm{~s}, 9 \mathrm{H}) .{ }^{13} \mathrm{C} \mathrm{NMR}(126 \mathrm{MHz}$, Chloroform-d) $\delta 167.26,155.08,147.30,134.61,131.80,131.75,126.91,125.61,125.32,115.66$, 113.83, 41.71, 35.03, 31.29. HRMS (MALDI): $\mathrm{m} / \mathrm{z}$ calculated 449.08316 for $\mathrm{C}_{19} \mathrm{H}_{20} \mathrm{ClF}_{3} \mathrm{~N}_{2} \mathrm{O}_{3} \mathrm{~S}$, found $449.08633\left([\mathrm{M}+\mathrm{H}]^{+}\right)$.

4-(tert-Butyl)-N-(2-fluoro-4-(methylsulfonamido)benzyl)benzamide (8): Preparation according to general procedure B using $N$-(4-amino-2-fluorobenzyl)-4-tert-butyl benzamide (53, $0.050 \mathrm{~g}, 0.17 \mathrm{mmol}$, $1.0 \mathrm{eq})$ and methanesulfonyl chloride $(73,0.095 \mathrm{~g}, 0.83 \mathrm{mmol}, 5.0 \mathrm{eq})$. The crude product was purified by column chromatography using $n$-hexane/EtOAc (4:3) as mobile phase to obtain 8 as a colorless solid (0.020 g, 32\%). M.p.: $215.5^{\circ} \mathrm{C} .{ }^{1} \mathrm{H} \mathrm{NMR}\left(500 \mathrm{MHz}\right.$, Methanol- $\left.d_{4}\right) \delta=7.80-7.76(\mathrm{~m}, 2 \mathrm{H}), 7.52-7.48(\mathrm{~m}$, $2 \mathrm{H}), 7.36(\mathrm{t}, J=8.4 \mathrm{~Hz}, 1 \mathrm{H}), 7.05(\mathrm{dd}, J=11.7,2.2 \mathrm{~Hz}, 1 \mathrm{H}), 7.02-6.97(\mathrm{dd}, J=8.4,2.2 \mathrm{~Hz}, 1 \mathrm{H}), 4.57(\mathrm{~s}$, $2 \mathrm{H}), 2.97(\mathrm{~s}, 3 \mathrm{H}), 1.34(\mathrm{~s}, 9 \mathrm{H}) .{ }^{13} \mathrm{C} N \mathrm{NM}\left(126 \mathrm{MHz}\right.$, Methanol- $\left.d_{4}\right) \delta=170.21,[163.31,161.36], 156.47$, [140.49, 140.41], 132.49, [131.66, 131.61], 128.22, 126.52, [122.69, 122.57], [116.51, 116.48], [108.03, 107.82], 52.56, 39.32, [37.96, 37.93], 30.53. HRMS (MALDI): $\mathrm{m} / \mathrm{z}$ calculated 401.13056 for $\mathrm{C}_{19} \mathrm{H}_{23} \mathrm{FN}_{2} \mathrm{O}_{3} \mathrm{SNa}$, found $401.12986\left([\mathrm{M}+\mathrm{Na}]^{+}\right)$.

4-(tert-Butyl)-N-(2,6-difluoro-4-(methylsulfonamido)benzyl)benzamide (9): Preparation according to general procedure B using $N$-(4-amino-2,6-difluorobenzyl)-4-(tert-butyl)benzamide (54, $0.300 \mathrm{~g}, 0.944$ $\mathrm{mmol}, 1.00 \mathrm{eq})$ and methanesulfonyl chloride $(73,0.541 \mathrm{~g}, 4.72 \mathrm{mmol}, 5.00 \mathrm{eq})$. The crude product was purified by column chromatography using $n$-hexane/EtOAc (4:3) as mobile phase to obtain 9 as a beige solid (0.184 g, 49\%). M.p.: $247.2{ }^{\circ} \mathrm{C} .{ }^{1} \mathrm{H}$ NMR $\left(500 \mathrm{MHz}\right.$, Methanol- $\left.d_{4}\right) \delta=7.75-7.71(\mathrm{~m}, 2 \mathrm{H})$, $7.48(\mathrm{~d}, J=8.5 \mathrm{~Hz}, 2 \mathrm{H}), 6.87(\mathrm{~d}, J=9.2 \mathrm{~Hz}, 2 \mathrm{H}), 4.58(\mathrm{~s}, 2 \mathrm{H}), 3.03(\mathrm{~s}, 3 \mathrm{H}), 1.33(\mathrm{~s}, 9 \mathrm{H}) .{ }^{13} \mathrm{C} \mathrm{NMR}(126 \mathrm{MHz}$, Methanol- $\left.d_{4}\right) \delta=170.01,[164.51,164.42,162.54,162.46], 156.39,[141.53,141.42], 132.48,128.27$, 126.46, [110.45, 110.29, 110.13], [103.20, 102.96], 39.63, 35.77, 32.58, 32.56, 32.53], 31.54. HRMS (MALDI): $\mathrm{m} / \mathrm{z}$ calculated 397.13920 for $\mathrm{C}_{19} \mathrm{H}_{22} \mathrm{~F}_{2} \mathrm{~N}_{2} \mathrm{O}_{3} \mathrm{~S}$, found $397.13908\left([\mathrm{M}+\mathrm{H}]^{+}\right)$.

4-(tert-Butyl)-N-(2,6-dichloro-4-(methylsulfonamido)benzyl)benzamide (10): Preparation according to general procedure B using $\mathrm{N}$-(4-amino-2,6-dichlorobenzyl)-4-(tert-butyl)benzamide (91, $0.065 \mathrm{~g}$, $0.19 \mathrm{mmol}, 1.0 \mathrm{eq})$ and methanesulfonyl chloride $(73,0.11 \mathrm{~g}, 0.93 \mathrm{mmol}, 5.0 \mathrm{eq})$. The crude product was purified by column chromatography using $n$-hexane/EtOAc (3:1) as mobile phase to obtain $\mathbf{1 0}$ as a yellow solid $(0.011 \mathrm{~g}, 14 \%)$. M.p.: $195.7^{\circ} \mathrm{C} .{ }^{1} \mathrm{H}$ NMR $\left(500 \mathrm{MHz}\right.$, Methanol- $\left.d_{4}\right) \delta=7.75-7.72(\mathrm{~m}, 2 \mathrm{H})$, $7.48-7.44(\mathrm{~m}, 2 \mathrm{H}), 7.31(\mathrm{~s}, 2 \mathrm{H}), 4.79(\mathrm{~s}, 2 \mathrm{H}), 3.03(\mathrm{~s}, 3 \mathrm{H}), 1.32(\mathrm{~s}, 9 \mathrm{H}) .{ }^{13} \mathrm{C}$ NMR (126 MHz, Methanol$\left.d_{4}\right) \delta=170.17,156.31,140.93,138.19,132.45,129.57,128.36,126.41,119.89,40.60,39.77,35.75$, 31.54. HRMS (MALDI): $\mathrm{m} / \mathrm{z}$ calculated 429.08010 for $\mathrm{C}_{19} \mathrm{H}_{23} \mathrm{Cl}_{2} \mathrm{~N}_{2} \mathrm{O}_{3} \mathrm{~S}$, found $429.08037\left([\mathrm{M}+\mathrm{H}]^{+}\right)$.

\section{4-(1-(Trifluoromethyl)cyclopropyl)-N-(2-chloro-4-(methylsulfonamido)benzyl)benzamide}

(11): Preparation according to general procedure $\mathrm{B}$ using $\mathrm{N}$-(4-amino-2-chlorobenzyl)-(4-(1(trifluoromethyl)cyclopropyl)benzamide $(55,0.104 \mathrm{~g}, 0.282 \mathrm{mmol}, 1.00$ equiv) and methanesulfonyl chloride $(73,0.323 \mathrm{~g}, 2.82 \mathrm{mmol}, 10.0 \mathrm{eq})$. The crude product was purified by column chromatography using $n$-hexane/EtOAc (1:1) to obtain 11 as a colorless solid (0.052 g, 41\%). M.p.: $168.8^{\circ} \mathrm{C} .{ }^{1} \mathrm{H}$ NMR $\left(500 \mathrm{MHz}, \mathrm{DMSO}-d_{6}\right) \delta=9.93(\mathrm{~s}, 1 \mathrm{H}), 9.04(\mathrm{~s}, 1 \mathrm{H}), 7.91(\mathrm{~d}, J=8.3 \mathrm{~Hz}, 2 \mathrm{H}), 7.57(\mathrm{~d}, J=8.1 \mathrm{~Hz}, 2 \mathrm{H}), 7.32$ $(\mathrm{d}, J=8.4 \mathrm{~Hz}, 1 \mathrm{H}), 7.26(\mathrm{~d}, J=2.1 \mathrm{~Hz}, 1 \mathrm{H}), 7.15(\mathrm{dd}, J=8.4,2.1 \mathrm{~Hz}, 1 \mathrm{H}), 4.48(\mathrm{~d}, J=5.7 \mathrm{~Hz}, 2 \mathrm{H}), 3.02(\mathrm{~s}$, $3 \mathrm{H}), 1.38(\mathrm{~s}, 2 \mathrm{H}), 1.17(\mathrm{~s}, 2 \mathrm{H}) .{ }^{13} \mathrm{C}$ NMR (126 MHz, Methanol- $\left.d_{4}\right) \delta=169.74,141.13,139.98,135.45$, 
134.93, 132.85, 132.52, 131.10, 128.53, [130.97, 128.80, 126.64, 124.47], 121.72, 119.59, 42.16, 39.37, [29.48, 29.21, 28.95, 28.68], [10.45, 10.44, 10.43, 10.42]. HRMS (MALDI): $\mathrm{m} / \mathrm{z}$ calculated 469.05710 for $\mathrm{C}_{19} \mathrm{H}_{18} \mathrm{ClF}_{3} \mathrm{~N}_{2} \mathrm{O}_{3} \mathrm{SNa}$, found $469.05613\left([\mathrm{M}+\mathrm{Na}]^{+}\right)$.

4-(Bicyclo[1.1.1]pentanyl)-N-(2-chloro-4-(methylsulfonamido)benzyl)benzamide (12): Preparation according to general procedure $\mathrm{B}$ using $\mathrm{N}$-(4-amino-2-chlorobenzyl)-4(bicyclo[1.1.1]pentanyl)benzamide (56, $0.040 \mathrm{~g}, 0.12 \mathrm{mmol}, 1.0 \mathrm{eq}$ ) and methanesulfonyl chloride (73, $0.14 \mathrm{~g}, 1.2 \mathrm{mmol}, 10 \mathrm{eq})$. The crude product was purified by column chromatography using $n$ hexane/EtOAc (3:1) as mobile phase to obtain 12 as a yellow solid $(0.021 \mathrm{~g}, 42 \%)$. M.p.: $180.5^{\circ} \mathrm{C} .{ }^{1} \mathrm{H}$ NMR (500 MHz, Chloroform-d) $\delta=7.71(\mathrm{~d}, J=8.3 \mathrm{~Hz}, 2 \mathrm{H}), 7.41(\mathrm{~d}, J=8.3 \mathrm{~Hz}, 1 \mathrm{H}), 7.32(\mathrm{~d}, J=2.3 \mathrm{~Hz}$, $1 \mathrm{H}), 7.27-7.24(\mathrm{~m}, 2 \mathrm{H}), 7.02(\mathrm{dd}, J=8.3,2.3 \mathrm{~Hz}, 1 \mathrm{H}), 4.66(\mathrm{~d}, J=6.1 \mathrm{~Hz}, 2 \mathrm{H}), 3.01(\mathrm{~s}, 3 \mathrm{H}), 2.57(\mathrm{~s}, 1 \mathrm{H})$, 2.09 (s, 6H). ${ }^{13} \mathrm{C}$ NMR $(126 \mathrm{MHz}$, Chloroform-d) $\delta=167.43,145.66,137.34,134.62,132.54,131.88$, 131.37, 126.88, 126.31, 121.12, 118.91, 52.20, 46.82, 41.58, 39.74, 26.89. HRMS (MALDI): $\mathrm{m} / \mathrm{z}$ calculated 405.10342 for $\mathrm{C}_{19} \mathrm{H}_{23} \mathrm{Cl}_{2} \mathrm{~N}_{2} \mathrm{O}_{3} \mathrm{~S}$, found $405.10359\left([\mathrm{M}+\mathrm{H}]^{+}\right)$.

4-(Trifluoromethyl)- $\mathrm{N}$-(2-chloro-4-(methylsulfonamido)benzyl)benzamide $\quad$ (13): Preparation according to general procedure B using $\mathrm{N}$-(4-amino-2-chlorobenzyl)-4-(trifluoromethyl)benzamide (57, $0.126 \mathrm{~g}, 0.383 \mathrm{mmol}, 1.00 \mathrm{eq})$ and methanesulfonyl chloride (73, $0.439 \mathrm{~g}, 3.83 \mathrm{mmol}, 10.0 \mathrm{eq})$. The crude product was purified by column chromatography using $n$-hexane/EtOAc (1:1) as mobile phase to obtain 13 as a colorless solid (0.042 g, 27\%). M.p.: $187.1^{\circ} \mathrm{C}$. ${ }^{1} \mathrm{H}$ NMR (500 MHz, Acetone- $\left.d_{6}\right) \delta=8.71$ $(\mathrm{s}, 1 \mathrm{H}), 8.40(\mathrm{t}, J=5.9 \mathrm{~Hz}, 1 \mathrm{H}), 8.07-8.00(\mathrm{~m}, 2 \mathrm{H}), 7.75-7.66(\mathrm{~m}, 2 \mathrm{H}), 7.36(\mathrm{~d}, J=8.4 \mathrm{~Hz}, 1 \mathrm{H}), 7.31(\mathrm{~d}$, $J=2.3 \mathrm{~Hz}, 1 \mathrm{H}), 7.15(\mathrm{dd}, J=8.4,2.3 \mathrm{~Hz}, 1 \mathrm{H}), 4.57(\mathrm{~d}, J=5.8 \mathrm{~Hz}, 2 \mathrm{H}), 2.93(\mathrm{~s}, 3 \mathrm{H}) .{ }^{13} \mathrm{C} \mathrm{NMR}(126 \mathrm{MHz}$, Acetone- $\left.d_{6}\right) \delta=166.50,139.65$, [139.12 139.11, 139.10, 139.09], 134.21, [133.47, 133.22, 132.96, 132.70], 132.63, 131.13, 128.98, [128.21, 126.05, 123.88, 121.72], [126.27, 126.24, 126.21, 126.18], 121.16, 119.34, 41.68, 39.68. HRMS (MALDI): $\mathrm{m} / \mathrm{z}$ calculated 407.04385 for $\mathrm{C}_{19} \mathrm{H}_{19} \mathrm{ClF}_{3} \mathrm{~N}_{2} \mathrm{O}_{3} \mathrm{~S}$, found $407.04364\left([\mathrm{M}+\mathrm{H}]^{+}\right)$

4-(Dimethylamino)- $\mathbf{N}$-(2-chloro-4-(methylsulfonamido)benzyl)benzamide $\quad$ (14): $\quad$ Preparation according to general procedure B using $\mathrm{N}$-(4-amino-2-chlorobenzyl)-4-(tert-butyl)benzamide (58, $0.204 \mathrm{~g}, 0.672 \mathrm{mmol}, 1.00 \mathrm{eq})$ and methanesulfonyl chloride (73, $0.385 \mathrm{~g}, 3.36 \mathrm{mmol}, 5.00 \mathrm{eq})$. The crude product was purified by column chromatography using EtOAc as mobile phase. The product was then obtained as a yellow solid $(0.064 \mathrm{~g}, 25 \%)$ by re-crystallisation from methylene chloride/ $n$-hexane and from water/acetone. M.p.: $189.9{ }^{\circ} \mathrm{C} .{ }^{1} \mathrm{H}$ NMR $\left(500 \mathrm{MHz}\right.$, Methanol- $\left.d_{4}\right) \delta=7.76(\mathrm{~d}, J=9.0 \mathrm{~Hz}, 2 \mathrm{H})$, $7.33(\mathrm{t}, J=5.5 \mathrm{~Hz}, 2 \mathrm{H}), 7.14(\mathrm{dd}, J=8.4,2.2 \mathrm{~Hz}, 1 \mathrm{H}), 6.73(\mathrm{~d}, J=9.0 \mathrm{~Hz}, 2 \mathrm{H}), 5.49(\mathrm{~s}, 1 \mathrm{H}), 4.59(\mathrm{~s}, 2 \mathrm{H})$, $3.02(\mathrm{~s}, 6 \mathrm{H}), 2.96(\mathrm{~s}, 3 \mathrm{H}) .{ }^{13} \mathrm{C}$ NMR $\left(126 \mathrm{MHz}\right.$, Methanol- $\left.d_{4}\right) \delta=170.50,154.38,139.71,134.72,133.51$, $130.77,129.89,121.74,121.54,119.63,112.13,41.90,40.22$, 39.32. HRMS (MALDI): $m / z$ calculated 380.08302 for $\mathrm{C}_{17} \mathrm{H}_{19} \mathrm{ClN}_{3} \mathrm{O}_{3} \mathrm{~S}$, found $380.08274\left([\mathrm{M}-\mathrm{H}]^{-}\right)$.

4-(Methoxy)-N-(2-chloro-4-(methylsulfonamido)benzyl)benzamide (15): Preparation according to general procedure B using $N$-(4-amino-2-chlorobenzyl)-4-(methoxy)benzamide (59, $0.200 \mathrm{~g}, 0.688$ $\mathrm{mmol}, 1.00 \mathrm{eq})$ and methanesulfonyl chloride $(73,0.394 \mathrm{~g}, 3.44 \mathrm{mmol}, 5.00 \mathrm{eq})$. The crude product was purified by column chromatography using $n$-hexane/EtOAc (2:3) as mobile phase to obtain $\mathbf{1 5}$ as a colorless solid (0.125 g, 18\%). M.p.: $160.2{ }^{\circ} \mathrm{C} .{ }^{1} \mathrm{H}$ NMR $\left(500 \mathrm{MHz}\right.$, Methanol- $\left.d_{4}\right) \delta=7.86-7.81(\mathrm{~m}$, $2 \mathrm{H}), 7.34(\mathrm{~d}, J=8.4 \mathrm{~Hz}, 1 \mathrm{H}), 7.32(\mathrm{~d}, J=2.2 \mathrm{~Hz}, 1 \mathrm{H}), 7.15(\mathrm{dd}, J=8.4,2.3 \mathrm{~Hz}, 1 \mathrm{H}), 7.01-6.96(\mathrm{~m}, 2 \mathrm{H})$, $4.60(\mathrm{~s}, 2 \mathrm{H}), 3.84(\mathrm{~s}, 3 \mathrm{H}), 2.96(\mathrm{~s}, 3 \mathrm{H}) .{ }^{13} \mathrm{C}$ NMR (126 MHz, Methanol- $\left.d_{4}\right) \delta=169.90,164.07,139.84$, 
134.83, 133.16, 130.94, 130.29, 127.36, 121.74, 119.61, 114.78, 55.93, 42.03, 39.35. HRMS (MALDI): $\mathrm{m} / \mathrm{z}$ calculated 369.06716 for $\mathrm{C}_{16} \mathrm{H}_{18} \mathrm{ClN}_{2} \mathrm{O}_{4} \mathrm{~S}$, found $369.06703\left([\mathrm{M}+\mathrm{H}]^{+}\right)$.

4-(Azetidin-1-yl)-N-(2-chloro-4-(methylsulfonamido)benzyl)benzamide (16): Preparation according to general procedure B using $N$-(4-amino-2-chlorobenzyl)-4-(azetidin-1-yl)benzamide (60, $0.055 \mathrm{~g}, 0.17$ $\mathrm{mmol}, 1.0 \mathrm{eq})$ and methanesulfonyl chloride $(\mathbf{7 3}, 0.099 \mathrm{~g}, 0.87 \mathrm{mmol}, 5.0 \mathrm{eq})$. The crude product was purified by column chromatography using $n$-hexane/EtOAc (1:3) as mobile phase to obtain 60 as a colorless solid (0.014 g, 21\%). M.p.: $161.2{ }^{\circ} \mathrm{C} .{ }^{1} \mathrm{H}$ NMR $\left(500 \mathrm{MHz}\right.$, DMSO- $\left.d_{6}\right) \delta=7.69(\mathrm{~d}, J=8.7 \mathrm{~Hz}, 2 \mathrm{H}$ ), $7.28-7.24(\mathrm{~m}, 2 \mathrm{H}), 7.14(\mathrm{dd}, J=8.4,2.2 \mathrm{~Hz}, 1 \mathrm{H}), 6.59(\mathrm{~d}, J=8.8 \mathrm{~Hz}, 2 \mathrm{H}), 4.43(\mathrm{~d}, J=5.8 \mathrm{~Hz}, 2 \mathrm{H}), 3.74$ $(\mathrm{t}, J=6.5 \mathrm{~Hz}, 2 \mathrm{H}), 3.03-3.01(\mathrm{~m}, 2 \mathrm{H}), 2.98(\mathrm{~s}, 3 \mathrm{H}), 1.99(\mathrm{q}, J=6.6 \mathrm{~Hz}, 2 \mathrm{H}) .{ }^{13} \mathrm{C}$ NMR $(126 \mathrm{MHz}, \mathrm{DMSO}-$ $\left.d_{6}\right) \delta=166.36,151.35,138.21,132.29,132.19,129.42,128.91,120.75,119.78,118.31,110.78,43.17$, 40.38, 39.88, 31.50. HRMS (MALDI): $\mathrm{m} / \mathrm{z}$ calculated 422.12997 for $\mathrm{C}_{20} \mathrm{H}_{25} \mathrm{ClN}_{3} \mathrm{O}_{3} \mathrm{~S}$, found 422.12986 $\left([\mathrm{M}+\mathrm{H}]^{+}\right)$.

4-(Pyrrolidine)- $\mathbf{N}$-(2-chloro-4-(methylsulfonamido)benzyl)benzamide (17): Preparation according to general procedure $\mathrm{B}$ using $\mathrm{N}$-(4-amino-2-chlorobenzyl)-4-(pyrrolidine)benzamide (61, $0.102 \mathrm{~g}, 0.031$ $\mathrm{mmol}, 1.00 \mathrm{eq})$ and methanesulfonyl chloride $(73,0.120 \mathrm{~mL}, 1.55 \mathrm{mmol}, 10.0 \mathrm{eq})$. The crude product was purified by column chromatography using $n$-hexane/EtOAc (1:2) to obtain $\mathbf{1 7}$ as a colorless solid (0.033 g, 26\%). M.p.: $167.2{ }^{\circ} \mathrm{C}^{1} \mathrm{H}$ NMR $\left(500 \mathrm{MHz}, \mathrm{DMSO}-\mathrm{d}_{6}\right) \delta=9.91(\mathrm{~s}, 1 \mathrm{H}), 8.60(\mathrm{~s}, 1 \mathrm{H}), 7.77$ (d, J= $8.8 \mathrm{~Hz}, 2 \mathrm{H}), 7.28(\mathrm{~d}, J=8.4 \mathrm{~Hz}, 1 \mathrm{H}), 7.25(\mathrm{~d}, J=2.1 \mathrm{~Hz}, 1 \mathrm{H}), 7.14(\mathrm{dd}, J=8.4,2.2 \mathrm{~Hz}, 1 \mathrm{H}), 6.54(\mathrm{~d}, J=8.9$ $\mathrm{Hz}, 2 \mathrm{H}), 4.44(\mathrm{~d}, J=5.5 \mathrm{~Hz}, 2 \mathrm{H}), 3.28(\mathrm{~s}, 4 \mathrm{H}), 3.01(\mathrm{~s}, 3 \mathrm{H}), 1.98-1.94(\mathrm{~m}, 4 \mathrm{H}) .{ }^{13} \mathrm{C} N M R(126 \mathrm{MHz}$, DMSO- $\left.d_{6}\right) \delta=166.38,149.61,138.20,132.30,132.19,129.43,128.81,119.97,119.76,118.29,110.59$, 47.23, 42.98, 36.56, 24.98. HRMS (MALDI): $\mathrm{m} / \mathrm{z}$ calculated 408.11432 for $\mathrm{C}_{19} \mathrm{H}_{23} \mathrm{ClN}_{3} \mathrm{O}_{3} \mathrm{~S}$, found $408.11361\left([\mathrm{M}+\mathrm{H}]^{+}\right)$.

4-(Piperidin-1-yl)-N-(2-chloro-4-(methylsulfonamido)benzyl)benzamide (18): Preparation according to general procedure B using $\mathrm{N}$-(4-amino-2-chlorobenzyl)-4-(piperdirin-1-yl)benzamide $(\mathbf{6 2}, 0.092 \mathrm{~g}$, $0.24 \mathrm{mmol}, 1.0 \mathrm{eq})$ and methanesulfonyl chloride $(73,0.27 \mathrm{~g}, 2.4 \mathrm{mmol}, 10 \mathrm{eq})$. The crude product was purified by column chromatography using $n$-hexane/EtOAc (1:1) as mobile phase to obtain 18 as a colorless solid (0.012 g, 7\%). M.p.: $157.6^{\circ} \mathrm{C} .{ }^{1} \mathrm{H}$ NMR $\left(500 \mathrm{MHz}\right.$, DMSO- $\left.d_{6}\right) \delta=9.93(\mathrm{~s}, 1 \mathrm{H}), 8.73(\mathrm{t}, \mathrm{J}=$ $5.8 \mathrm{~Hz}, 1 \mathrm{H}), 7.79(\mathrm{~d}, J=8.6 \mathrm{~Hz}, 2 \mathrm{H}), 7.28(\mathrm{~d}, J=8.4 \mathrm{~Hz}, 1 \mathrm{H}), 7.26(\mathrm{~d}, J=2.2 \mathrm{~Hz}, 1 \mathrm{H}), 7.14(\mathrm{dd}, J=8.4,2.3$ $\mathrm{Hz}, 1 \mathrm{H}), 7.00(\mathrm{~d}, J=8.5 \mathrm{~Hz}, 2 \mathrm{H}), 4.45(\mathrm{~d}, J=5.7 \mathrm{~Hz}, 2 \mathrm{H}), 3.29(\mathrm{~m}, 4 \mathrm{H}), 3.00(\mathrm{~s}, 3 \mathrm{H}), 1.64-1.54(\mathrm{~m}, 6 \mathrm{H})$. ${ }^{13} \mathrm{C}$ NMR $\left(126 \mathrm{MHz}\right.$, DMSO- $\left.d_{6}\right) \delta=166.55,138.71,132.69,132.53,129.92,129.19,128.63,120.62$, $120.25,118.77,114.59,49.27,46.02,40.28,25.22,24.21$. HRMS (MALDI): $\mathrm{m} / \mathrm{z}$ calculated 422.12997 for $\mathrm{C}_{20} \mathrm{H}_{25} \mathrm{ClN}_{3} \mathrm{O}_{3} \mathrm{~S}$, found $422.12986\left([\mathrm{M}+\mathrm{H}]^{+}\right)$.

4-(Morpholin-4-yl)-N-(2-chloro-4-(methylsulfonamido)benzyl)benzamide $\quad$ (19): Preparation according to general procedure B using $N$-(4-amino-2-chlorobenzyl)-4-(morpholin-4-yl)benzamide (63, $0.101 \mathrm{~g}, 0.292 \mathrm{mmol}, 1.00 \mathrm{eq}$ ) and methanesulfonyl chloride (73, $0.335 \mathrm{~g}, 2.92 \mathrm{mmol}, 10.0 \mathrm{eq})$. The crude product was purified by column chromatography using $n$-hexane/EtOAc (1:1) as mobile phase to obtain 19 as a colorless solid (0.070 g, 57\%). M.p.: $186.2^{\circ} \mathrm{C}^{1} \mathrm{H} \mathrm{NMR}\left(500 \mathrm{MHz}, \mathrm{DMSO}-d_{6}\right) \delta=9.92$ (s, $1 \mathrm{H}), 8.74(\mathrm{t}, J=5.8 \mathrm{~Hz}, 1 \mathrm{H}), 7.85-7.75(\mathrm{~m}, 2 \mathrm{H}), 7.29(\mathrm{~d}, J=8.4 \mathrm{~Hz}, 1 \mathrm{H}), 7.26(\mathrm{~d}, J=2.2 \mathrm{~Hz}, 1 \mathrm{H}), 7.15$ $(\mathrm{dd}, J=8.4,2.2 \mathrm{~Hz}, 1 \mathrm{H}), 7.00-6.94(\mathrm{~m}, 2 \mathrm{H}), 4.45(\mathrm{~d}, J=5.7 \mathrm{~Hz}, 2 \mathrm{H}), 3.73(\mathrm{~m}, 4 \mathrm{H}), 3.21(\mathrm{~m}, 4 \mathrm{H}) .{ }^{13} \mathrm{C} \mathrm{NMR}$ $\left(126 \mathrm{MHz}, \mathrm{DMSO}-d_{6}\right) \delta=166.14,153.11,138.31,132.31,132.08,129.55,128.67,123.69,119.83$, 118.34, 113.49, 65.98, 47.42, 40.39, 39.77. HRMS (MALDI): $\mathrm{m} / \mathrm{z}$ calculated 424.10923 for $\mathrm{C}_{19} \mathrm{H}_{23} \mathrm{ClN}_{3} \mathrm{O}_{4} \mathrm{~S}$, found $424.10844\left([\mathrm{M}+\mathrm{H}]^{+}\right)$. 
4-(Trifluoromethoxy)-N-(2-chloro-4-(methylsulfonamido)benzyl)benzamide $\quad$ (20): Preparation according to general procedure B using $\mathrm{N}$-(4-amino-2-chlorobenzyl)-4-(trifluoromethoxy)benzamide (64, $0.300 \mathrm{~g}, 0.870 \mathrm{mmol}, 1.00 \mathrm{eq})$ and methanesulfonyl chloride $(73,0.498 \mathrm{~g}, 4.35 \mathrm{mmol}, 5.00 \mathrm{eq})$. The crude product was purified by column chromatography using $n$-hexane/EtOAc (1:1) as mobile phase to obtain 20 as a colorless solid $(0.211 \mathrm{~g}, 57 \%)$. M.p.: $185.8^{\circ} \mathrm{C} .{ }^{1} \mathrm{H} N M R\left(500 \mathrm{MHz}\right.$, Methanol- $\left.d_{4}\right)$ $\delta=7.97(\mathrm{~d}, J=8.8 \mathrm{~Hz}, 2 \mathrm{H}), 7.37(\mathrm{~m}, 3 \mathrm{H}), 7.33(\mathrm{~d}, J=2.3 \mathrm{~Hz}, 1 \mathrm{H}), 7.17(\mathrm{dd}, J=8.4,2.3 \mathrm{~Hz}, 1 \mathrm{H}), 4.62(\mathrm{~s}$, $2 \mathrm{H}), 2.97(\mathrm{~s}, 3 \mathrm{H}) .{ }^{13} \mathrm{C}$ NMR $\left(126 \mathrm{MHz}\right.$, Methanol- $\left.d_{4}\right) \delta=168.83$, [152.84, 152.83, 152.81, 152.80], 140.04, 134.99, 134.31, 132.73, 131.22, 130.63, [124.86, 122.82, 120.78, 118.74], 121.78, 121.71, $119.58,42.24$, 39.38. HRMS (MALDI): $\mathrm{m} / z$ calculated 423.03828 for $\mathrm{C}_{19} \mathrm{H}_{22} \mathrm{~F}_{2} \mathrm{~N}_{2} \mathrm{O}_{3} \mathrm{~S}$, found 423.03877 $\left([\mathrm{M}+\mathrm{H}]^{+}\right)$.

4-(Ethoxy)- $\mathbf{N}$-(2-chloro-4-(methylsulfonamido)benzyl)benzamide (21): Preparation according to general procedure B using $\mathrm{N}$-(4-amino-2-chlorobenzyl)-4-ethoxybenzamide $(65,0.216 \mathrm{~g}, 0.709 \mathrm{mmol}$, $1.00 \mathrm{eq})$ and methanesulfonyl chloride $(73,0.406 \mathrm{~g}, 3.54 \mathrm{mmol}, 5.00 \mathrm{eq})$. The crude product was purified by column chromatography using $n$-hexane/EtOAc (3:4) as mobile phase to obtain $\mathbf{2 1}$ as a colorless solid (0.084 g, 23\%). M.p.: $164.4{ }^{\circ} \mathrm{C}^{1} \mathrm{H}$ NMR (500 MHz, DMSO- $\left.d_{6}\right) \delta=9.91(\mathrm{~s}, 1 \mathrm{H}), 8.83$ (t, $J=$ $5.7 \mathrm{~Hz}, 1 \mathrm{H}), 7.87(\mathrm{~d}, J=8.9 \mathrm{~Hz}, 2 \mathrm{H}), 7.31(\mathrm{~d}, J=8.4 \mathrm{~Hz}, 1 \mathrm{H}), 7.26(\mathrm{~d}, J=2.2 \mathrm{~Hz}, 1 \mathrm{H}), 7.15(\mathrm{dd}, J=8.4,2.2$ $\mathrm{Hz}, 1 \mathrm{H}), 7.05-6.93(\mathrm{~m}, 2 \mathrm{H}), 4.46(\mathrm{~d}, J=5.7 \mathrm{~Hz}, 2 \mathrm{H}), 4.09$ (q, J = $7.0 \mathrm{~Hz}, 2 \mathrm{H}), 3.01(\mathrm{~s}, 3 \mathrm{H}), 1.34$ (t, J = 7.0 $\mathrm{Hz}, 3 \mathrm{H}) .{ }^{13} \mathrm{C}$ NMR $\left(126 \mathrm{MHz}\right.$, DMSO- $\left.d_{6}\right) \delta=166.37,161.47,138.81,132.78,132.32,130.06,129.65$, $126.63,120.24,118.76,114.41,63.79,40.59,39.92,15.02$. HRMS (MALDI): $m / z$ calculated 383.08268 for $\mathrm{C}_{17} \mathrm{H}_{20} \mathrm{ClN}_{2} \mathrm{O}_{4} \mathrm{~S}$, found $383.08226\left([\mathrm{M}+\mathrm{H}]^{+}\right)$.

4-(Isopropyloxy)- $\mathbf{N}$-(2-chloro-4-(methylsulfonamido)benzyl)benzamide (22): Preparation according to general procedure $B$ using $N$-(4-amino-2-chlorobenzyl)-4-isopropyloxybenzamide $(66,0.500 \mathrm{~g}, 1.57$ $\mathrm{mmol}, 1.00 \mathrm{eq})$ and methanesulfonyl chloride $(73,0.898 \mathrm{~g}, 7.84 \mathrm{mmol}, 5.00 \mathrm{eq})$. The crude product was purified by column chromatography using $n$-hexane/EtOAc (3:4) as mobile phase to obtain 22 as a colorless solid (0.429 g, 69\%). M.p.: $173.2{ }^{\circ} \mathrm{C}^{1} \mathrm{H}$ NMR $\left(600 \mathrm{MHz}\right.$, DMSO- $\left.d_{6}\right) \delta=9.91(\mathrm{~s}, 1 \mathrm{H}), 8.82(\mathrm{t}, J$ $=5.8 \mathrm{~Hz}, 1 \mathrm{H}), 7.88-7.84(\mathrm{~m}, 2 \mathrm{H}), 7.31(\mathrm{~d}, J=8.4 \mathrm{~Hz}, 1 \mathrm{H}), 7.26(\mathrm{t}, J=1.8 \mathrm{~Hz}, 1 \mathrm{H}), 7.15(\mathrm{dt}, J=8.4,1.6$ $\mathrm{Hz}, 1 \mathrm{H}), 7.01-6.94(\mathrm{~m}, 2 \mathrm{H}), 4.70(\mathrm{spt}, J=6.0 \mathrm{~Hz}, 1 \mathrm{H}), 4.46(\mathrm{~d}, J=5.7 \mathrm{~Hz}, 2 \mathrm{H}), 3.01(\mathrm{~s}, 3 \mathrm{H}), 1.28(\mathrm{~d}, J=$ $6.1 \mathrm{~Hz}, 6 \mathrm{H}) .{ }^{13} \mathrm{C}$ NMR $\left(151 \mathrm{MHz}\right.$, DMSO- $\left.d_{6}\right) \delta=165.89,159.97,138.28,132.27,131.84,129.54,129.16$, 125.96, 119.78, 118.29, 114.87, 69.38, 40.05, 39.43, 21.68. HRMS (MALDI): $m / z$ calculated 397.09833 for $\mathrm{C}_{18} \mathrm{H}_{22} \mathrm{ClN}_{2} \mathrm{O}_{4} \mathrm{~S}$, found $397.09813\left([\mathrm{M}+\mathrm{H}]^{+}\right)$.

4-(2-Hydroxypropan-2-yl)- $\mathrm{N}$-(2-chloro-4-(methylsulfonamido)benzyl)benzamide (23): $\mathrm{N}$-(4-Amino2-chlorobenzyl)-4-(2-hydroxypropan-2-yl)benzamide (67, $0.103 \mathrm{~g}, 0.323 \mathrm{mmol}, 1.00 \mathrm{eq})$ was dissolved in a mixture of THF $(5 \mathrm{~mL})$ and pyridine $(5 \mathrm{~mL})$. Then methanesulfonyl chloride $(73,0.111 \mathrm{~g}, 0.969$ mmol, 3.00 eq) was added dropwise at $0^{\circ} \mathrm{C}$ and the reaction mixture was stirred at $0^{\circ} \mathrm{C}$ for 8 hours. Aqueous hydrochloric acid $(10 \%, 15 \mathrm{~mL})$ was added and the phases were separated. The aqueous layer was extracted with ethyl acetate $(3 \times 30 \mathrm{~mL})$. The combined organic layers were dried over $\mathrm{Na}_{2} \mathrm{SO}_{4}$ and the solvent was removed in vacuum. Further purification was performed by column chromatography using $n$-hexane/EtOAc (5:3) as mobile phase to obtain 23 as a colorless solid $(0.006 \mathrm{~g}, 5 \%)$. M.p.: $191.5^{\circ} \mathrm{C} .{ }^{1} \mathrm{H}$ NMR $\left(500 \mathrm{MHz}\right.$, Methanol- $\left.d_{4}\right) \delta=7.85-7.82(\mathrm{~m}, 2 \mathrm{H}), 7.59(\mathrm{~d}, J=8.4 \mathrm{~Hz}, 2 \mathrm{H}), 7.37-7.33$ $(\mathrm{m}, 2 \mathrm{H}), 7.16(\mathrm{dd}, J=8.4,2.3 \mathrm{~Hz}, 1 \mathrm{H}), 4.62(\mathrm{~s}, 2 \mathrm{H}), 2.96(\mathrm{~s}, 3 \mathrm{H}), 1.54(\mathrm{~s}, 6 \mathrm{H}) .{ }^{13} \mathrm{C} N M R(126 \mathrm{MHz}$, Methanol- $\left.d_{4}\right) \delta=168.80,153.67,138.51,133.49,129.62,127.96,126.87,126.60,124.48,120.35$, $118.23,71.51,40.70,37.98,30.39$. HRMS (MALDI): $\mathrm{m} / \mathrm{z}$ calculated 419.08028 for $\mathrm{C}_{18} \mathrm{H}_{21} \mathrm{ClN}_{2} \mathrm{O}_{4} \mathrm{SNa}$, found $419.08020\left([\mathrm{M}+\mathrm{Na}]^{+}\right)$. 
4-(2-Methoxypropan-2-yl)-N-(2-chloro-4-(methylsulfonamido)benzyl)benzamide (24): Preparation according to general procedure $\mathrm{B}$ using $\mathrm{N}$-(4-amino-2-chlorobenzyl)-4-(2-methoxypropan-2yl)benzamide $(68,0.100 \mathrm{~g}, 0.315 \mathrm{mmol}, 1.00 \mathrm{eq})$ and methanesulfonyl chloride $(73,0.180 \mathrm{~g}, 1.57 \mathrm{mmol}$, 5.00 eq). The crude product was purified by column chromatography using $n$-hexane/EtOAc (2:3) as mobile phase to obtain 24 as a colorless solid (0.059 g, $46 \%$ ). M.p.: $182.7^{\circ} \mathrm{C}^{1} \mathrm{H}$ NMR (500 MHz, DMSO$\left.d_{6}\right) \delta=9.93(\mathrm{~s}, 1 \mathrm{H}), 8.98(\mathrm{t}, J=5.8 \mathrm{~Hz}, 1 \mathrm{H}), 7.91-7.87(\mathrm{~m}, 2 \mathrm{H}), 7.50-7.46(\mathrm{~m}, 2 \mathrm{H}), 7.32(\mathrm{~d}, J=8.4 \mathrm{~Hz}$, $1 \mathrm{H}), 7.27$ (d, J = 2.2 Hz, 1H), $7.16(\mathrm{dd}, J=8.4,2.2 \mathrm{~Hz}, 1 \mathrm{H}), 4.49$ (d, J = 5.7 Hz, 2H), $3.02(\mathrm{~s}, 3 \mathrm{H}), 2.99$ (s, $3 \mathrm{H}), 1.46(\mathrm{~s}, 6 \mathrm{H}) .{ }^{13} \mathrm{C}$ NMR $(126 \mathrm{MHz} \text {, DMSO-d })_{6} \delta=166.22,149.60,138.39,132.58,132.34,131.65$, $129.59,127.32,125.54,119.75,118.28,76.26,50.04,40.59,39.90,27.57$. HRMS (MALDI): $\mathrm{m} / \mathrm{z}$ calculated 433.09474 for $\mathrm{C}_{19} \mathrm{H}_{23} \mathrm{ClN}_{2} \mathrm{O}_{4} \mathrm{SNa}$, found $433.09593\left([\mathrm{M}+\mathrm{Na}]^{+}\right)$.

4-(3-Hydroxyoxetan-3-yl)-N-(2-chloro-4-(methylsulfonamido)benzyl)benzamide (25): $\quad 3-(4-((2-$ Chloro-4-(methylsulfonamido)benzyl)carbamoyl)phenyl)oxetan-3-yl acetate (96, 0.044 g, $0.097 \mathrm{mmol}$, $1.00 \mathrm{eq})$ was solved in a mixture of THF $(7 \mathrm{~mL})$ and EtOH $(3 \mathrm{~mL})$. Then LiOH $(0.030 \mathrm{~g}, 1.26 \mathrm{mmol}, 13.0$ eq) dissolved in $\mathrm{H}_{2} \mathrm{O}(3 \mathrm{~mL}$ ) was added and the mixture was stirred at room temperature for $5 \mathrm{~h}$. The solution was then neutralized with aqueous hydrochloric acid (10\%) and the solvents were evaporated in vacuum. $\mathrm{H}_{2} \mathrm{O}(20 \mathrm{~mL})$ and EtOAc $(20 \mathrm{~mL})$ were added, phases were separated and the aqueous layer was extracted with EtOAc $(3 \times 20 \mathrm{~mL})$. The combined organic layers were dried over $\mathrm{Na}_{2} \mathrm{SO}_{4}$ and the solvent was removed in vacuum. Further purification was performed by column chromatography using EtOAc as mobile phase to obtain 25 as a colorless solid (0.019 g, 48\%). M.p.: $201.4^{\circ} \mathrm{C} .{ }^{1} \mathrm{H}$ NMR (500 $\mathrm{MHz}$, Methanol- $\left.d_{4}\right) \delta=7.88-7.83(\mathrm{~m}, 2 \mathrm{H}), 7.54(\mathrm{~d}, J=8.4 \mathrm{~Hz}, 2 \mathrm{H}), 7.36(\mathrm{~d}, J=8.3 \mathrm{~Hz}, 1 \mathrm{H}), 7.33(\mathrm{~d}, J=$ $2.3 \mathrm{~Hz}, 1 \mathrm{H}), 7.16(\mathrm{dd}, J=8.4,2.4 \mathrm{~Hz}, 1 \mathrm{H}), 4.61(\mathrm{~s}, 2 \mathrm{H}), 4.11(\mathrm{~d}, J=12.6 \mathrm{~Hz}, 1 \mathrm{H}), 3.87(\mathrm{~d}, J=12.6 \mathrm{~Hz}, 1 \mathrm{H})$, $3.16(\mathrm{~d}, J=5.4 \mathrm{~Hz}, 1 \mathrm{H}), 2.97(\mathrm{~s}, 3 \mathrm{H}), 2.75(\mathrm{~d}, J=5.4 \mathrm{~Hz}, 1 \mathrm{H}) .{ }^{13} \mathrm{C} \mathrm{NMR}\left(126 \mathrm{MHz}\right.$, Methanol- $\left.d_{4}\right) \delta=169.83$, 143.56, 139.90, 134.88, 134.67, 132.89, 131.07, 128.45, 127.58, 121.70, 119.58, 86.99, 64.81, 42.11, 40.40. HRMS (MALDI): $\mathrm{m} / \mathrm{z}$ calculated 411.07760 for $\mathrm{C}_{18} \mathrm{H}_{20} \mathrm{ClN}_{2} \mathrm{O}_{5} \mathrm{~S}$, found $411.07727\left([\mathrm{M}+\mathrm{H}]^{+}\right)$.

4-(1,1-Difluoroethyl)-N-(2-chloro-4-(cyclopropylsulfonamido)benzyl)benzamide (26): Preparation according to general procedure B using $N$-(4-amino-2-chlorobenzyl)-4-(1,1-difluoroethyl)benzamide (69, $0.075 \mathrm{~g}, 0.23 \mathrm{mmol} 1.0 \mathrm{eq})$ and methanesulfonyl chloride (73, $0.079 \mathrm{~g}, 0.62 \mathrm{mmol}, 3.0 \mathrm{eq})$. The crude product was purified by column chromatography using $n$-hexane/EtOAc (1:1) as mobile phase to obtain 26 as a yellow solid (0.037 g, 40\%). M.p.: $169.2{ }^{\circ} \mathrm{C} .{ }^{1} \mathrm{H}$ NMR $\left(500 \mathrm{MHz}\right.$, DMSO- $\left.d_{6}\right) \delta=9.94$ (s, $1 \mathrm{H}), 9.13(\mathrm{t}, J=5.8 \mathrm{~Hz}, 1 \mathrm{H}), 8.00(\mathrm{~d}, J=8.1 \mathrm{~Hz}, 2 \mathrm{H}), 7.68(\mathrm{~d}, J=8.1 \mathrm{~Hz}, 2 \mathrm{H}), 7.34(\mathrm{~d}, J=8.4 \mathrm{~Hz}, 1 \mathrm{H}), 7.27$ $(\mathrm{d}, J=2.2 \mathrm{~Hz}, 1 \mathrm{H}), 7.16(\mathrm{dd}, J=8.4,2.2 \mathrm{~Hz}, 1 \mathrm{H}), 4.50(\mathrm{~d}, J=5.6 \mathrm{~Hz}, 2 \mathrm{H}), 3.02(\mathrm{~s}, 3 \mathrm{H}), 1.99(\mathrm{t}, J=18.9 \mathrm{~Hz}$, $3 H) .{ }^{13} \mathrm{C}$ NMR $(126 \mathrm{MHz}$, DMSO-d 6 ) $\delta=165.77,[140.23,140.02,139.81], 138.52,135.66,132.50$, 131.43, 129.80, 127.79, 124.86, 124.81, 124.77, [124.07, 122.18, 120.29], 119.80, 118.31, [25.30, 25.07, 24.84]. HRMS (MALDI): $\mathrm{m} / z$ calculated 403.06892 for $\mathrm{C}_{17} \mathrm{H}_{18} \mathrm{ClF}_{2} \mathrm{~N}_{2} \mathrm{O}_{3} \mathrm{~S}$, found 403.06892 $\left([\mathrm{M}+\mathrm{H}]^{+}\right)$.

4-(2,2,2-Trifluoroethyl)- $\mathrm{N}$-(2-fluoro-4-(methylsulfonamido)benzyl)benzamide (27): Preparation according to general procedure $\mathrm{B}$ using $\mathrm{N}$-(4-amino-2-chlorobenzyl)-4-(2,2,2-trifluoroethyl)benzamide (70, $0.072 \mathrm{~g}, 0.21 \mathrm{mmol}, 1.0 \mathrm{eq})$ and methanesulfonyl chloride $(73,0.12 \mathrm{~g}, 1.1 \mathrm{mmol}, 5.0 \mathrm{eq})$. The crude product was purified by column chromatography using $n$-hexane/EtOAc (3:1) as mobile phase to obtain 27 as a colorless solid (0.039 g, 44\%). M.p.: $149.7^{\circ} \mathrm{C} .{ }^{1} \mathrm{H}$ NMR $\left(500 \mathrm{MHz}\right.$, Methanol- $\left.d_{4}\right) \delta=7.88-7.84$ $(\mathrm{m}, 2 \mathrm{H}), 7.45(\mathrm{~d}, J=8.0 \mathrm{~Hz}, 2 \mathrm{H}), 7.36(\mathrm{~d}, J=8.4 \mathrm{~Hz}, 1 \mathrm{H}), 7.33(\mathrm{~d}, J=2.2 \mathrm{~Hz}, 1 \mathrm{H}), 7.16(\mathrm{dd}, J=8.4,2.3 \mathrm{~Hz}$, $1 \mathrm{H}), 4.62(\mathrm{~s}, 2 \mathrm{H}), 3.57(\mathrm{q}, J=11.1 \mathrm{~Hz}, 2 \mathrm{H}), 2.96(\mathrm{~s}, 3 \mathrm{H}) .{ }^{13} \mathrm{C}$ NMR $\left(126 \mathrm{MHz}\right.$, Methanol- $\left.d_{4}\right) \delta=169.79$, 139.92, [135.79, 135.77, 135.74, 135.72], 135.12, 134.91, 132.86, 131.61, 131.07, 128.68, [130.60 
128.40, 126.21, $124.01 \mathrm{~Hz}$ ] 121.72, 119.60, 42.14, [40.61, 40.37, 40.14, 39.90], 39.37. HRMS (MALDI): $\mathrm{m} / \mathrm{z}$ calculated 421.05950 for $\mathrm{C}_{17} \mathrm{H}_{17} \mathrm{ClF}_{3} \mathrm{~N}_{2} \mathrm{O}_{3} \mathrm{~S}$, found $421.05910\left([\mathrm{M}+\mathrm{H}]^{+}\right)$.

4-(Pentafluoroethyl)-N-(2-fluoro-4-(methylsulfonamido)benzyl)benzamide $\quad$ (28): $\quad$ Preparation according to general procedure $\mathrm{B}$ using $\mathrm{N}$-(4-amino-2-chlorobenzyl)-4-(pentafluoroethyl)benzamide (71, $0.050 \mathrm{~g}, 0.13 \mathrm{mmol}, 1.0 \mathrm{eq}$ ) and methanesulfonyl chloride (73, $0.076 \mathrm{~g}, 0.66 \mathrm{mmol}, 5.0 \mathrm{eq})$. The crude product was purified by column chromatography using $n$-hexane/EtOAc (2:1) as mobile phase to obtain 28 as a colorless solid (0.019 g, 32\%). M.p.: $158.3^{\circ} \mathrm{C} .{ }^{1} \mathrm{H}$ NMR (500 MHz, DMSO- $\left.d_{6}\right) \delta=9.94$ $(\mathrm{s}, 1 \mathrm{H}), 9.23(\mathrm{t}, J=5.7 \mathrm{~Hz}, 1 \mathrm{H}), 8.15-8.10(\mathrm{~m}, 2 \mathrm{H}), 7.83(\mathrm{~d}, J=8.3 \mathrm{~Hz}, 2 \mathrm{H}), 7.37(\mathrm{~d}, J=8.4 \mathrm{~Hz}, 1 \mathrm{H}), 7.28$ $(\mathrm{d}, J=2.2 \mathrm{~Hz}, 1 \mathrm{H}), 7.17$ (dd, $J=8.4,2.2 \mathrm{~Hz}, 1 \mathrm{H}), 4.52(\mathrm{~d}, J=5.6 \mathrm{~Hz}, 2 \mathrm{H}), 3.02(\mathrm{~s}, 3 \mathrm{H}) .{ }^{13} \mathrm{C} \mathrm{NMR}(126 \mathrm{MHz}$, DMSO- $\left.d_{6}\right) \delta=165.27,138.52,138.11,132.48,131.14,129.82$, [129.58, 129.40, 129.21], 128.27, $[126.56,126.51,126.46],\{[120.12,119.81,119.49],[117.85,117.53,117.22]\}, 119.71,118.20$, $\{[115.60,115.30,114.99,114.69],[112.98,113.28,112.98,112.68],[111.56,111.26,110.96,110.66]\}$, 40.33, 40.11. HRMS (MALDI): $\mathrm{m} / z$ calculated 457.04066 for $\mathrm{C}_{17} \mathrm{H}_{15} \mathrm{ClF}_{5} \mathrm{~N}_{2} \mathrm{O}_{3} \mathrm{~S}$, found 457.03975 $\left([\mathrm{M}+\mathrm{H}]^{+}\right)$.

4-(Pentafluorosulfanyl)- $\mathrm{N}$-(2-chloro-4-(methylsulfonamido)benzyl)benzamide (29): Preparation according to general procedure $\mathrm{B}$ using $\mathrm{N}$-(4-amino-2-chlorobenzyl)-4-(pentafluorosulfanyl)benzamide (72, $0.092 \mathrm{~g}, 0.24 \mathrm{mmol}, 1.0 \mathrm{eq})$ and methanesulfonyl chloride $(73,0.272 \mathrm{~g}, 2.38 \mathrm{mmol}, 10.0 \mathrm{eq})$. The crude product was purified by column chromatography using $n$-hexane/EtOAc (1:1) as mobile phase to obtain 29 as a colorless solid (0.074 g, 67\%). M.p.: $180.7^{\circ} \mathrm{C}$. ${ }^{1} \mathrm{H}$ NMR (500 MHz, Acetone- $\left.d_{6}\right) \delta=8.81$ $(\mathrm{s}, 1 \mathrm{H}), 8.54(\mathrm{t}, J=5.8 \mathrm{~Hz}, 1 \mathrm{H}), 8.18-8.10(\mathrm{~m}, 2 \mathrm{H}), 8.02-7.93(\mathrm{~m}, 2 \mathrm{H}), 7.48(\mathrm{~d}, J=8.4 \mathrm{~Hz}, 1 \mathrm{H}), 7.42(\mathrm{~d}$, $J=2.3 \mathrm{~Hz}, 1 \mathrm{H}), 7.26(\mathrm{dd}, J=8.4,2.3 \mathrm{~Hz}, 1 \mathrm{H}), 4.68(\mathrm{~d}, J=5.8 \mathrm{~Hz}, 2 \mathrm{H}), 3.04(\mathrm{~s}, 3 \mathrm{H}) .{ }^{13} \mathrm{C} \mathrm{NMR}(126 \mathrm{MHz}$, Acetone) $\delta=165.98$, [156.36, 156.22, 156.08, 155.95, 155.80], 139.69, 138.83, 134.24, 132.52, 131.17, 129.13, [127.10, 127.06, 127.02, 126.97, 126.94], 121.16, 119.34, 41.73, 39.69. HRMS (MALDI): $\mathrm{m} / \mathrm{z}$ calculated 465.01273 for $\mathrm{C}_{15} \mathrm{H}_{14} \mathrm{ClF}_{5} \mathrm{~N}_{2} \mathrm{O}_{3} \mathrm{~S}_{2}$, found $465.01161\left([\mathrm{M}+\mathrm{H}]^{+}\right)$.

4-(Aminomethyl)-3-fluoroaniline (31): A solution of $\mathrm{LiAlH}_{4}$ in THF (1 M, $18.4 \mathrm{~mL}, 18.4 \mathrm{mmol}, 2.50$ eq) was cooled to $0^{\circ} \mathrm{C}$ and diluted with THF (20 mL). 4-Amino-2-fluorobenzonitrile (79, $1.00 \mathrm{~g}, 7.35 \mathrm{mmol}$, $1.00 \mathrm{eq})$ in THF ( $5 \mathrm{~mL}$ ) was slowly added to the mixture. After evolution of $\mathrm{H}_{2}$ had ceased, the mixture was allowed to warm to room temperature and then refluxed for $16 \mathrm{~h}$. After cooling to room temperature, the mixture was diluted with $10 \mathrm{~mL}$ of THF and then cooled to $0^{\circ} \mathrm{C}$. Aqueous $\mathrm{NaOH}(10 \%$, $1 \mathrm{~mL})$ and $\mathrm{H}_{2} \mathrm{O}(1.8 \mathrm{~mL})$ were added dropwise. The colorless precipitate was filtered off through celite and washed with THF $(15 \mathrm{~mL})$. Evaporation of the organic solvents from the filtrate yielded $\mathbf{3 1}$ as a yellow oil $(0.816 \mathrm{~g}, 79 \%) .{ }^{1} \mathrm{H}$ NMR $\left(300 \mathrm{MHz}\right.$, DMSO- $\left.d_{6}\right) \delta=7.03(\mathrm{t}, J=8.6 \mathrm{~Hz}, 1 \mathrm{H}), 6.35-6.24(\mathrm{~m}, 2 \mathrm{H})$, $5.18(\mathrm{~s}, 2 \mathrm{H}), 3.55(\mathrm{~s}, 2 \mathrm{H}) .{ }^{13} \mathrm{C}$ NMR $\left(75 \mathrm{MHz}, \mathrm{DMSO}-d_{6}\right) \delta=[162.49,159.31],[149.23,149.08],[129.83$, 129.73], [117.16, 116.95], [109.66, 109.63], [100.13, 99.80], [38.79, 38.74].

4-(Aminomethyl)-3,5-difluoroaniline (32): $\mathrm{LiAlH}_{4}(1 \mathrm{M}$ in THF, $4.87 \mathrm{~mL}, 4.87 \mathrm{mmol}, 2.50 \mathrm{eq}$ ) was cooled to $0^{\circ} \mathrm{C}$ and diluted with $20 \mathrm{~mL}$ of THF. 4-Amino-2,6-difluorobenzonitrile (80, $0.300 \mathrm{~g}, 1.95 \mathrm{mmol}, 1.00$ eq) in THF ( $5 \mathrm{~mL}$ ) was slowly added to the mixture. After evolution of $\mathrm{H}_{2}$ had ceased, the mixture was allowed to warm to room temperature and then refluxed for $16 \mathrm{~h}$. After cooling to room temperature, the mixture was diluted with $10 \mathrm{~mL}$ of THF and then cooled to $0^{\circ} \mathrm{C}$. Aqueous $\mathrm{NaOH}(10 \%, 1 \mathrm{~mL})$ and $\mathrm{H}_{2} \mathrm{O}(2 \mathrm{~mL})$ were added dropwise. The colorless precipitate was filtered through celite and washed with THF (15 mL). Evaporation of the organic solvents from the filtrate yielded as yellow oil $(0.185 \mathrm{~g}$, $60 \%) .{ }^{1} \mathrm{H}$ NMR $\left(300 \mathrm{MHz}, \mathrm{DMSO}-d_{6}\right) \delta=6.16(\mathrm{~d}, J=2.0 \mathrm{~Hz}, 1 \mathrm{H}), 6.13(\mathrm{~d}, J=1.9 \mathrm{~Hz}, 1 \mathrm{H}), 5.56(\mathrm{~s}, 2 \mathrm{H})$, 
$3.53(\mathrm{~s}, 2 \mathrm{H}) .{ }^{13} \mathrm{C}$ NMR $\left(75 \mathrm{MHz}\right.$, DMSO- $\left.d_{6}\right) \delta[163.28,163.11,160.09,159.92,[149.78,149.58,149.39]$, [96.29, 95.91], 96.16, 96.04, [32.88, 32.83, 32.79], 30.41.

4-((1-Trifluouromethyl)cycloprop-1-yl)-benzoic acid (45): $\mathrm{Pd}(\mathrm{OAc})_{2}(0.010 \mathrm{~g}, 0.045 \mathrm{mmol}, 0.03 \mathrm{eq})$ and Xantphos $(0.010 \mathrm{~g}, 0.045 \mathrm{mmol}, 0.03 \mathrm{eq})$ were dissolved in DMF $(10 \mathrm{~mL})$. Afterwards formic acid $(0.400$ $\mathrm{mL}, 10.7 \mathrm{mmol}, 7.00 \mathrm{eq}$ ) and 1-bromo-4-(1-(trifluoromethyl)cycloprop-1-yl)benzene (81, $0.400 \mathrm{~g}, 1.51$ $\mathrm{mmol}, 1.00 \mathrm{eq})$ were added dropwise. Then EDC. $\mathrm{HCl}(0.058 \mathrm{~g}, 0.30 \mathrm{mmol}, 0.20 \mathrm{eq})$ and triethylamine $(0.305 \mathrm{~g}, 3.02 \mathrm{mmol}, 2.00 \mathrm{eq})$ were added and the mixture was stirred at $50^{\circ} \mathrm{C}$ for $20 \mathrm{~h}$. After cooling to room temperature, aqueous hydrochloric acid $(10 \%, 10 \mathrm{~mL})$ was added and the mixture was extracted with EtOAc $(3 \times 30 \mathrm{~mL})$. The combined organic layers were extracted with a saturated aqueous solution of $\mathrm{Na}_{2} \mathrm{CO}_{3}(3 \times 30 \mathrm{~mL})$. Then, the combined aqueous layers were brought to $\mathrm{pH} 1$ with concentrated aqueous hydrochloric acid and extracted with EtOAc $(3 \times 30 \mathrm{~mL})$. The combined organic layers were dried over $\mathrm{Na}_{2} \mathrm{SO}_{4}$ and the solvent was evaporated in vacuum to obtain 45 as a beige solid $(0.177 \mathrm{~g}, 51 \%) .{ }^{1} \mathrm{H} N M R\left(300 \mathrm{MHz}, \mathrm{DMSO}-d_{6}\right) \delta=12.89(\mathrm{~s}, 1 \mathrm{H}), 7.98-7.90(\mathrm{~m}, 2 \mathrm{H}), 7.62-7.54(\mathrm{~m}, 2 \mathrm{H})$, $1.44-1.33(\mathrm{~m}, 2 \mathrm{H}), 1.22-1.14(\mathrm{~m}, 2 \mathrm{H}) .{ }^{13} \mathrm{C} \mathrm{NMR}\left(75 \mathrm{MHz}, \mathrm{DMSO}-d_{6}\right) \delta=166.87,140.02,131.18$, 130.86, 129.41, [131.78, 128.15, 124.52, 121.15], [28.16, 27.75, 27.32, 26.88], [9.81, 9.77, 9.74, 9.71].

4-(1,1-Difluoroethyl)benzoic acid (46): $\mathrm{Pd}(\mathrm{OAc})_{2}(0.015 \mathrm{~g}, 0.067 \mathrm{mmol}, 0.030 \mathrm{eq})$ and Xantphos (0.039 g, $0.067 \mathrm{mmol}, 0.030 \mathrm{eq})$ were dissolved in DMF $(10 \mathrm{~mL})$. Afterwards formic acid $(0.597 \mathrm{~mL}, 15.8 \mathrm{mmol}$, $7.00 \mathrm{eq})$ and 1-bromo-4-(1,1-difluoroethyl)benzene $(82,0.500 \mathrm{~g}, 2.26 \mathrm{mmol}, 1.00 \mathrm{eq})$ were added dropwise. Then EDC. $\mathrm{HCl}(0.058 \mathrm{~g}, 0.30 \mathrm{mmol}, 0.20 \mathrm{eq})$ and triethylamine $(0.305 \mathrm{~g}, 3.02 \mathrm{mmol}, 2.00 \mathrm{eq})$ were added and the mixture was stirred at $50^{\circ} \mathrm{C}$ for $20 \mathrm{~h}$. After cooling to room temperature aqueous hydrochloric acid $(10 \%, 10 \mathrm{~mL})$ was added and the mixture was extracted three times with EtOAc $(3 \times 30$ $\mathrm{mL})$. The combined organic layers were extracted with a saturated aqueous solution of $\mathrm{Na}_{2} \mathrm{CO}_{3}(3 \times 30$ $\mathrm{mL}$ ). Then, the combined aqueous layers were brought to $\mathrm{pH} 1$ with concentrated aqueous hydrochloric acid and extracted with EtOAc $(3 \times 30 \mathrm{~mL})$. The combined organic layers were dried over $\mathrm{Na}_{2} \mathrm{SO}_{4}$ and the solvent was evaporated in vacuum to obtain 46 as a colorless solid $(0.202 \mathrm{~g}, 48 \%) .{ }^{1} \mathrm{H}$ NMR $\left(300 \mathrm{MHz}\right.$, Methanol- $\left.d_{4}\right) \delta=8.10(\mathrm{~d}, J=8.0 \mathrm{~Hz}, 2 \mathrm{H}), 7.63(\mathrm{~d}, J=8.1 \mathrm{~Hz}, 2 \mathrm{H}), 1.93(\mathrm{t}, J=18.4 \mathrm{~Hz}$, $3 \mathrm{H}) .{ }^{13} \mathrm{C}$ NMR $\left(75 \mathrm{MHz}\right.$, Methanol- $\left.d_{4}\right) \delta=168.93$, [144.12, 143.77, 143.42], 133.42, 130.99, [125.97, $125.89,125.81]$, [125.98, 122.82, 119.66], [26.27, 25.88, 25.49].

4-(2,2,2-Trifluoroethyl)benzoic acid (47): $\mathrm{Pd}(\mathrm{OAc})_{2}(0.018 \mathrm{~g}, 0.063 \mathrm{mmol}, 0.03 \mathrm{eq})$ and Xantphos $(0.044$ $\mathrm{g}, 0.063 \mathrm{mmol}, 0.030 \mathrm{eq})$ were dissolved in DMF $(10 \mathrm{~mL})$. Afterwards formic acid $(0.552 \mathrm{~mL}, 14.6 \mathrm{mmol}$, $7.00 \mathrm{eq})$ and 1-bromo-4-(2,2,2-trifluoroethyl)benzene $(83,0.500 \mathrm{~g}, 2.09 \mathrm{mmol}, 1.00 \mathrm{eq})$ were added dropwise. Then EDC. $\mathrm{HCl}(0.201 \mathrm{~g}, 1.05 \mathrm{mmol}, 0.500 \mathrm{eq})$ and triethylamine $(0.423 \mathrm{~g}, 4.18 \mathrm{mmol}, 2.00$ eq) were added and the mixture was stirred at $50^{\circ} \mathrm{C}$ for $20 \mathrm{~h}$. After cooling to room temperature aqueous hydrochloric acid $(10 \%, 10 \mathrm{~mL})$ was added and the mixture was extracted with EtOAc $(3 \times 30$ $\mathrm{mL})$. The combined organic layers were extracted with a saturated aqueous solution of $\mathrm{Na}_{2} \mathrm{CO}_{3}(3 \times 30$ $\mathrm{mL}$ ). Then, the combined aqueous layers were brought to $\mathrm{pH} 1$ with concentrated aqueous hydrochloric acid and extracted with EtOAc $(3 \times 30 \mathrm{~mL})$. The combined organic layers were dried over $\mathrm{Na}_{2} \mathrm{SO}_{4}$ and the solvent was evaporated in vacuum to obtain 47 as a colorless solid $(0.337 \mathrm{~g}, 79 \%) .{ }^{1} \mathrm{H}$ $\operatorname{NMR}\left(300 \mathrm{MHz}\right.$, DMSO- $\left.d_{6}\right) \delta=7.95(\mathrm{~d}, J=8.2 \mathrm{~Hz}, 2 \mathrm{H}), 7.48(\mathrm{~d}, J=8.0 \mathrm{~Hz}, 2 \mathrm{H}), 3.75(\mathrm{q}, J=11.6 \mathrm{~Hz}, 2 \mathrm{H})$. ${ }^{13} \mathrm{C}$ NMR $\left(75 \mathrm{MHz}\right.$, DMSO- $\left.d_{6}\right) \delta=167.02$, [135.43, 135.39, 135.35, 135.31], [131.68, 128.01, 124.34, 120.67] 130.55, 130.50, 129.52, [38.90, 38.52, 38.14, 37.76]. 
4-Pentafluoroethylbenzoic acid (48): 4-(Pentafluoroethyl)benzonitrile (84, $0.300 \mathrm{~g}, 1.36 \mathrm{mmol}, 1.00$ eq) was dissolved in EtOH $(10 \mathrm{~mL}) . \mathrm{KOH}(0.382 \mathrm{~g}, 6.80 \mathrm{mmol}, 5.00 \mathrm{eq})$ was dissolved in $\mathrm{H}_{2} \mathrm{O}(10 \mathrm{~mL})$. Subsequently, both reaction mixtures were combined and stirred at $100^{\circ} \mathrm{C}$ for $48 \mathrm{~h}$. After cooling to room temperature aqueous hydrochloric acid $(10 \%, 10 \mathrm{~mL})$ was added and the mixture was extracted with EtOAc $(3 \times 30 \mathrm{~mL})$. The combined organic layers were extracted with a saturated aqueous solution of $\mathrm{Na}_{2} \mathrm{CO}_{3}(3 \times 30 \mathrm{~mL})$. Then the combined aqueous layers were brought to $\mathrm{pH} 1$ with concentrated aqueous hydrochloric acid and extracted with EtOAc $(3 \times 30 \mathrm{~mL})$. The combined organic layers were dried over $\mathrm{Na}_{2} \mathrm{SO}_{4}$ and the solvent was evaporated in vacuum to obtain $\mathbf{4 8}$ as a brown solid $(0.118 \mathrm{~g}$, 36\%). ${ }^{1} \mathrm{H}$ NMR $\left(300 \mathrm{MHz}\right.$, Methanol- $\left.d_{4}\right) \delta=9.09(\mathrm{~d}, J=8.0 \mathrm{~Hz}, 2 \mathrm{H}), 8.79(\mathrm{~d}, J=8.0 \mathrm{~Hz}, 2 \mathrm{H}) .{ }^{13} \mathrm{C} \mathrm{NMR}(75$ $\mathrm{MHz}$, Methanol- $\left.d_{4}\right) \delta=170.38,138.72,134.80,[132.38,132.07,131.74], 128.96$, [127.47, 127.39, 127.31], [121.70, 118.17, 114.64].

4-(2-Hydroxypropan-2-yl)benzoic acid (49): 4-Isopropylbenzoic acid (85, $0.700 \mathrm{~g}, 4.26 \mathrm{mmol}, 1.00 \mathrm{eq}$ ) was dissolved in $\mathrm{H}_{2} \mathrm{O}(15 \mathrm{~mL})$ and pyridine $(15 \mathrm{~mL})$. After stirring the solution at $100^{\circ} \mathrm{C}$ for $1 \mathrm{~h}, \mathrm{KMnO}_{4}$ $(2.02 \mathrm{~g}, 12.8 \mathrm{mmol}, 3.00 \mathrm{eq})$ was added. The resulting mixture was stirred at $100^{\circ} \mathrm{C}$ for $16 \mathrm{~h}$, then quenched with an aqueous $\mathrm{NaOH}$ solution $(10 \%, 50 \mathrm{~mL})$ and filtered. The filtrate was brought to $\mathrm{pH} 1$ using concentrated aqueous hydrochloric acid and extracted with EtOAc $(3 \times 30 \mathrm{~mL})$. The combined organic layers were dried over $\mathrm{Na}_{2} \mathrm{SO}_{4}$ and the solvent was evaporated in vacuum. Further purification was performed by column chromatography using hexane/acetone (9:2) as mobile phase to obtain 49 as a colorless solid $(0.352 \mathrm{~g}, 46 \%) .{ }^{1} \mathrm{H}$ NMR $\left(400 \mathrm{MHz}\right.$, DMSO- $\left.d_{6}\right) \delta=7.87(\mathrm{~d}, J=8.2 \mathrm{~Hz}, 2 \mathrm{H}), 7.57(\mathrm{~d}, J=$ $8.2 \mathrm{~Hz}, 2 \mathrm{H}), 5.16(\mathrm{~s}, 1 \mathrm{H}), 1.43(\mathrm{~s}, 6 \mathrm{H}) .{ }^{13} \mathrm{C} \mathrm{NMR}\left(101 \mathrm{MHz}\right.$, DMSO- $\left.d_{6}\right) \delta=167.31,155.50,128.92,128.57$, $124.69,70.74,31.66$.

4-(2-Methoxypropan-2-yl)benzoic acid (50): 4-(2-Hydroxypropan-2-yl)benzoic acid (49, 0.400 g, 2.22 mmol, 1.00 eq) was dissolved in methanol $(10 \mathrm{~mL})$ and concetrated $\mathrm{H}_{2} \mathrm{SO}_{4}(0.1 \mathrm{~mL})$ was added. The resulting mixture was stirred at $80^{\circ} \mathrm{C}$ for $2 \mathrm{~h}$. After cooling to room temperature, a saturated solution of $\mathrm{NaHCO}_{3}(20 \mathrm{~mL})$ was added, methanol was removed under vacuum and the remaining aqueous phase was extracted with EtOAc $(3 \times 30 \mathrm{~mL})$. The combined organic layers were dried over $\mathrm{Na}_{2} \mathrm{SO}_{4}$ and the solvent was evaporated in vacuum. The crude product was dissolved in a mixture of methanol (15 $\mathrm{mL})$ and $\mathrm{H}_{2} \mathrm{O}(4 \mathrm{~mL})$, $\mathrm{LiOH}(0.261 \mathrm{~g}, 11.1 \mathrm{mmol}, 5.00 \mathrm{eq})$ was added and the resulting mixture was stirred at $80^{\circ} \mathrm{C}$ for $3 \mathrm{~h}$. After cooling to room temperature, aqueous hydrochloric acid $(10 \%, 20 \mathrm{~mL})$ was added, methanol was removed under vacuum and the remaining aqueous phase was extracted with EtOAc ( $3 \times 30 \mathrm{~mL})$. The combined organic layers were dried over $\mathrm{Na}_{2} \mathrm{SO}_{4}$ and the solvent was evaporated in vacuum. Further purification was performed by column chromatography using hexane/acetone (1:2) as mobile phase to obtain 50 as a colorless solid $(0.352 \mathrm{~g}, 82 \%) .{ }^{1} \mathrm{H}$ NMR $\left(300 \mathrm{MHz}\right.$, Methanol- $\left.d_{4}\right) \delta=$ $8.05(\mathrm{~d}, J=8.5 \mathrm{~Hz}, 2 \mathrm{H}), 7.57(\mathrm{~d}, J=8.5 \mathrm{~Hz}, 2 \mathrm{H}), 3.13(\mathrm{~s}, 3 \mathrm{H}), 1.58(\mathrm{~s}, 6 \mathrm{H}) .{ }^{13} \mathrm{C}$ NMR $(75 \mathrm{MHz}$, Methanol$\left.d_{4}\right) \delta=168.10,152.82,130.49,129.83,126.90,77.93,50.98,28.07,28.06$.

4-(Azetidin-1-yl)benzoic acid (51): Azetidine hydrochloride $(0.834 \mathrm{~g}, 8.92 \mathrm{mmol}, 5.00 \mathrm{eq})$ and $\mathrm{K}_{2} \mathrm{CO}_{3}$ (2.48 g, $10.7 \mathrm{mmol}, 6.00 \mathrm{eq})$ were suspended in DMSO (10 mL) and stirred at room temperature for 30 minutes. After a slow dropwise addition of ethyl 4-fluorobenzoate $(87,0.300 \mathrm{~g}, 1.78 \mathrm{mmol}, 1.00 \mathrm{eq})$ the mixture was stirred at $180^{\circ} \mathrm{C}$ for 72 hours. After cooling to room temperature the mixture was diluted with $\mathrm{H}_{2} \mathrm{O}(10 \mathrm{~mL})$. $\mathrm{LiOH}(0.214 \mathrm{~g}, 8.90 \mathrm{mmol}, 5.00 \mathrm{eq})$ and $\mathrm{EtOH}(5 \mathrm{~mL})$ were added and the mixture was stirred at $100^{\circ} \mathrm{C}$ for $16 \mathrm{~h}$. After cooling to room temperature aqueous hydrochloric acid $(10 \%, 10 \mathrm{~mL})$ was added and the mixture was extracted with EtOAc $(3 \times 30 \mathrm{~mL})$. The combined organic layers were extracted with a saturated aqueous solution of $\mathrm{Na}_{2} \mathrm{CO}_{3}(3 \times 30 \mathrm{~mL})$. The combined aqueous 
layers were then brought to $\mathrm{pH} 1$ with concentrated aqueous hydrochloric acid and extracted with EtOAc $(3 \times 30 \mathrm{~mL})$. The combined organic layers were dried over $\mathrm{Na}_{2} \mathrm{SO}_{4}$ and the solvent was evaporated in vacuum. Further purification was performed by column chromatography using hexane/EtOAc (3:1) to obtain 51 as a yellow solid $(0.131 \mathrm{~g}, 42 \%) .{ }^{1} \mathrm{H}$ NMR $\left(300 \mathrm{MHz}\right.$, Methanol- $\left.d_{4}\right) \delta=7.82-7.74(\mathrm{~m}, 2 \mathrm{H})$, $6.63-6.55(\mathrm{~m}, 2 \mathrm{H}), 3.67(\mathrm{t}, J=6.4 \mathrm{~Hz}, 2 \mathrm{H}), 3.35-3.31(\mathrm{~m}, 2 \mathrm{H}), 2.06(\mathrm{q}, J=6.6 \mathrm{~Hz}, 2 \mathrm{H}) .{ }^{13} \mathrm{C}$ NMR $(75$ MHz Methanol- $\left.d_{4}\right) \delta=170.75,154.42,132.79,118.36,112.11,43.31,40.97,33.05$.

$\mathbf{N}$-(4-Amino-2-fluorobenzyl)-4-(tert-butyl)benzamide (53): Preparation according to general procedure A using 4-(tert-butyl)benzoic acid (33, $0.240 \mathrm{~g}, 1.35 \mathrm{mmol}, 1.00 \mathrm{eq}), 1$-(4-amino-1fluorophenyl)methanamine (31, $0.245 \mathrm{~g}, 1.75 \mathrm{mmol}, 1.30 \mathrm{eq})$, EDC.HCl (0.310 g, $1.62 \mathrm{mmol}, 1.20 \mathrm{eq})$ and 4-DMAP $(0.016 \mathrm{~g}, 0.14 \mathrm{mmol}, 0.10 \mathrm{eq})$. The crude product was purified by column chromatography using $\mathrm{n}$-hexane/EtOAc (3:2) as mobile phase to obtain $\mathbf{5 3}$ as a colorless solid (0.063 g, 16\%). ${ }^{1} \mathrm{H}$ NMR (300 MHz, Methanol- $\left.d_{4}\right) \delta=7.79-7.72(\mathrm{~m}, 2 \mathrm{H}), 7.50-7.45(\mathrm{~m}, 2 \mathrm{H}), 7.09$ (dd, J = 9.0, 8.0 Hz, 1H), 6.48 $-6.38(\mathrm{~m}, 2 \mathrm{H}), 4.47(\mathrm{~s}, 2 \mathrm{H}), 1.33(\mathrm{~s}, 9 \mathrm{H}) .{ }^{13} \mathrm{C}$ NMR $\left(75 \mathrm{MHz}\right.$, Methanol- $\left.d_{4}\right) \delta=170.09$, [164.70, 161.48], 156.24, [150.71, 150.56], 132.76, [131.67, 131.59], 128.21, 126.43, [114.92, 114.71], [111.90, 111.87], [102.76, 102.43], [38.15, 38.10], 35.74, 31.55.

$\mathbf{N}$-(4-Amino-2,6-difluorobenzyl)-4-(tert-butyl)benzamide (54): Preparation according to general procedure A using 4-(tert-butyl)benzoic acid (33, $0.240 \mathrm{~g}, 1.35 \mathrm{mmol}, 1.00 \mathrm{eq}), 1$-(4-amino-2,6difluorophenyl)methanamine (32, $0.277 \mathrm{~g}, 1.75 \mathrm{mmol}, 1.30 \mathrm{eq}), \mathrm{EDC} \cdot \mathrm{HCl}(0.310 \mathrm{~g}, 1.62 \mathrm{mmol}, 1.20 \mathrm{eq})$ and 4-DMAP $(0.016 \mathrm{~g}, 0.14 \mathrm{mmol}, 0.10 \mathrm{eq})$. The crude product was purified by column chromatography using $\mathrm{n}$-hexane/EtOAc (6:1) as mobile phase to obtain $\mathbf{5 4}$ as a beige oil $(0.100 \mathrm{~g}, 22 \%) .{ }^{1} \mathrm{H}$ NMR (400 $\mathrm{MHz}$, Methanol- $\left.d_{4}\right) \delta=7.72(\mathrm{~d}, J=8.5 \mathrm{~Hz}, 2 \mathrm{H}), 7.47(\mathrm{~d}, J=8.5 \mathrm{~Hz}, 2 \mathrm{H}), 6.23(\mathrm{~d}, J=10.1 \mathrm{~Hz}, 2 \mathrm{H}$ ), 4.48 (s, $2 \mathrm{H}), 1.32(\mathrm{~s}, 9 \mathrm{H}) .{ }^{13} \mathrm{C}$ NMR (101 MHz, Methanol- $\left.d_{4}\right) \delta=169.99,[165.23,165.12,162.81,162.70]$, 156.20, [151.83, 151.69], 132.70, 128.25, 126.40, [102.32, 102.12, 101.92], [98.21, 97.93], 35.73, [32.59, 32.55, 32.52], 31.54.

$\mathbf{N}$-(4-Amino-2-chlorobenzyl)-4-(1-(trifluoromethyl)cyclopropyl)benzamide $\quad$ (55): $\quad$ Preparation according to general procedure A using 4-(1-(trifluoromethyl)cyclopropyl)benzoic acid $(45,0.153 \mathrm{~g}$, $0.665 \mathrm{mmol}, 1.00 \mathrm{eq}$ ), 1-(4-amino-1-chlorophenyl)methanamine (30, $0.312 \mathrm{~g}, 1.99 \mathrm{mmol}, 3.00 \mathrm{eq}$ ), $\mathrm{EDC} \cdot \mathrm{HCl}(0.382 \mathrm{~g}, 1.99 \mathrm{mmol}, 3.00 \mathrm{eq})$ and 4-DMAP $(0.081 \mathrm{~g}, 0.67 \mathrm{mmol}, 1.0 \mathrm{eq})$. The crude product was purified by column chromatography using $n$-hexane/EtOAc (4:3) as mobile phase to obtain $\mathbf{5 5}$ as a yellow solid $(0.125 \mathrm{~g}, 51 \%) .{ }^{1} \mathrm{H}$ NMR $\left(300 \mathrm{MHz}\right.$, Acetone- $\left.d_{6}\right) \delta=7.95-7.90(\mathrm{~m}, 2 \mathrm{H}), 7.57(\mathrm{~d}, J=8.1$ $\mathrm{Hz}, 2 \mathrm{H}), 7.15(\mathrm{~d}, J=8.3 \mathrm{~Hz}, 1 \mathrm{H}), 6.72(\mathrm{~d}, J=2.3 \mathrm{~Hz}, 1 \mathrm{H}), 6.58(\mathrm{dd}, J=8.3,2.3 \mathrm{~Hz}, 1 \mathrm{H}), 4.85(\mathrm{~s}, 1 \mathrm{H}), 4.54$ $(\mathrm{d}, J=5.6 \mathrm{~Hz}, 2 \mathrm{H}), 1.39$ (dd, $J=6.9,5.0 \mathrm{~Hz}, 2 \mathrm{H}), 1.18-1.12(\mathrm{~m}, 2 \mathrm{H}) .{ }^{13} \mathrm{C}$ NMR $\left(75 \mathrm{MHz}, \mathrm{DMSO}-d_{6}\right) \delta=$ 166.17, 149.60, [138.79, 138.78], [135.95, 132.33, 128.71, 125.08], 134.96, 132.91, 131.39, 130.23, $127.94,122.79,114.07,113.09,40.60,[28.58,28.14,27.70,27.26]$, [10.21, 10.18, 10.15, 10.12].

$\mathbf{N}$-(4-Amino-2-chlorobenzyl)-4-(bicyclo[1.1.1]pentanyl)benzamide (56): Preparation according to general procedure A using 4-(bicyclo[1.1.1]pentanyl)benzoic acid (34, $0.080 \mathrm{~g}, 0.43 \mathrm{mmol}, 1.0 \mathrm{eq})$, 1(4-amino-1-chlorophenyl)methanamine (30, $0.20 \mathrm{~g}, 1.3 \mathrm{mmol}, 3.0 \mathrm{eq}), \mathrm{EDC} \cdot \mathrm{HCl}(0.098 \mathrm{~g}, 0.51 \mathrm{mmol}$, $1.2 \mathrm{eq})$ and 4-DMAP $(0.005 \mathrm{~g}, 0.04 \mathrm{mmol}, 0.1 \mathrm{eq})$. The crude product was purified by column chromatography using n-hexane/EtOAc (7:2) as mobile phase to obtain $\mathbf{5 6}$ as a colorless solid (0.040 $\mathrm{g}$, 58\%). ${ }^{1} \mathrm{H}$ NMR (400 MHz, DMSO- $\left.d_{6}\right) \delta=7.84(\mathrm{~d}, J=8.3 \mathrm{~Hz}, 2 \mathrm{H}), 7.27(\mathrm{~d}, J=8.2 \mathrm{~Hz}, 2 \mathrm{H}), 7.00(\mathrm{~d}, J=8.3$ $\mathrm{Hz}, 1 \mathrm{H}), 6.62(\mathrm{~d}, J=2.2 \mathrm{~Hz}, 1 \mathrm{H}), 6.48(\mathrm{dd}, J=8.3,2.2 \mathrm{~Hz}, 1 \mathrm{H}), 4.38(\mathrm{~s}, 2 \mathrm{H}), 2.56(\mathrm{~s}, 1 \mathrm{H}), 2.07(\mathrm{~s}, 6 \mathrm{H}) .{ }^{13} \mathrm{C}$ 
NMR $\left(101 \mathrm{MHz}, \mathrm{DMSO}-d_{6}\right) \delta=170.59,149.02,144.03,132.47,130.49,128.93,128.09,126.47,124.89$, $124.82,122.50,51.61,40.66,38.68,27.12$.

$\mathbf{N}$-(4-Amino-2-chlorobenzyl)-4-(trifluoromethyl)benzamide (57): Preparation according to general procedure A using 4-(trifluoromethyl)benzoic acid $(35,0.200 \mathrm{~g}, 0.912 \mathrm{mmol}, 1.00 \mathrm{eq}), 1$-(4-amino-1chlorophenyl)methanamine (30, $0.429 \mathrm{~g}, 2.74 \mathrm{mmol}, 3.00 \mathrm{eq})$, EDC. $\mathrm{HCl}(0.525 \mathrm{~g}, 2.74 \mathrm{mmol}, 3.00 \mathrm{eq})$ and 4-DMAP $(0.111 \mathrm{~g}, 0.912 \mathrm{mmol}, 1.00 \mathrm{eq})$. The crude product was purified by column chromatography using n-hexane/EtOAc (4:3) as mobile phase to obtain 57 as a yellow solid $(0.126 \mathrm{~g}$, 42\%). ${ }^{1} \mathrm{H}$ NMR (300 MHz, DMSO- $\left.d_{6}\right) \delta=9.02(\mathrm{t}, J=5.2 \mathrm{~Hz}, 1 \mathrm{H}), 8.07(\mathrm{~d}, J=8.2 \mathrm{~Hz}, 2 \mathrm{H}), 7.84(\mathrm{~d}, J=8.2$ $\mathrm{Hz}, 2 \mathrm{H}), 7.04(\mathrm{~d}, J=8.3 \mathrm{~Hz}, 1 \mathrm{H}), 6.63(\mathrm{~d}, J=2.2 \mathrm{~Hz}, 1 \mathrm{H}), 6.49(\mathrm{dd}, J=8.3,2.1 \mathrm{~Hz}, 1 \mathrm{H}), 5.30(\mathrm{~s}, 2 \mathrm{H}), 4.40$ $(d, J=5.5 \mathrm{~Hz}, 2 \mathrm{H}) .{ }^{13} \mathrm{C} \mathrm{NMR}\left(75 \mathrm{MHz}\right.$, Acetone- $\left.d_{6}\right) \delta=166.13,150.09,139.50,134.43,[133.56,133.13$, 132.70, 132.28], 131.67, [130.44, 126.84, 123.23, 119.63], 128.95, [126.21, 126.16, 126.11, 126.06], $124.07,115.21,113.88,41.81$.

$\mathbf{N}$-(4-Amino-2-chlorobenzyl)-4-(dimethylamino)benzamide (58): Preparation according to general procedure A using 4-(dimethylamino)benzoic acid (45, $0.300 \mathrm{~g}, 1.82 \mathrm{mmol}, 1.00 \mathrm{eq}), 1$-(4-amino-1chlorophenyl)methanamine (30, $0.853 \mathrm{~g}, 5.45 \mathrm{mmol}, 3.00 \mathrm{eq})$, EDC. $\mathrm{HCl}(0.487 \mathrm{~g}, 2.54 \mathrm{mmol}, 1.40 \mathrm{eq})$ and 4-DMAP $(0.044 \mathrm{~g}, 0.36 \mathrm{mmol}, 0.20 \mathrm{eq})$. The crude product was purified by column chromatography using $\mathrm{n}$-hexane/EtOAc $(2: 1)+2 \%$ triethylamine as mobile phase to obtain $\mathbf{5 5}$ as a brown solid $(0.125 \mathrm{~g}$, 33\%). ${ }^{1} \mathrm{H}$ NMR $\left(250 \mathrm{MHz}, \mathrm{DMSO}-d_{6}\right) \delta=8.42(\mathrm{t}, J=5.7 \mathrm{~Hz}, 1 \mathrm{H}), 7.76(\mathrm{~d}, J=9.0 \mathrm{~Hz}, 2 \mathrm{H}), 6.98(\mathrm{~d}, J=8.3$ $\mathrm{Hz}, 1 \mathrm{H}), 6.70(\mathrm{~d}, J=9.0 \mathrm{~Hz}, 2 \mathrm{H}), 6.61(\mathrm{~d}, J=2.2 \mathrm{~Hz}, 1 \mathrm{H}), 6.47(\mathrm{dd}, J=8.3,2.3 \mathrm{~Hz}, 1 \mathrm{H}), 5.24(\mathrm{~s}, 2 \mathrm{H}), 4.34$ $(d, J=5.7 \mathrm{~Hz}, 2 \mathrm{H}) .{ }^{13} \mathrm{C}$ NMR $\left(75 \mathrm{MHz}\right.$, DMSO- $\left.d_{6}\right) \delta=166.25,152.23,148.96,132.34,129.62,128.71$, $123.13,121.02,113.65,112.70,110.88,40.82,39.87$.

$\mathbf{N}$-(4-Amino-2-chlorobenzyl)-4-methoxybenzamide (59): Preparation according to general procedure A using 4-methoxybenzoic acid (37, $0.350 \mathrm{~g}, 2.30 \mathrm{mmol}, 1.00 \mathrm{eq}), \quad 1$-(4-amino-1chlorophenyl)methanamine (30, $0.468 \mathrm{~g}, 2.99 \mathrm{mmol}, 1.30 \mathrm{eq})$, EDC. $\mathrm{HCl}(0.572 \mathrm{~g}, 2.99 \mathrm{mmol}, 1.30 \mathrm{eq})$ and 4-DMAP $(0.020 \mathrm{~g}, 0.23 \mathrm{mmol}, 0.10 \mathrm{eq})$. The crude product was purified by column chromatography using $\mathrm{n}$-hexane/EtOAc (3:2) as mobile phase to obtain 59 as a colorless solid $(0.577 \mathrm{~g}, 87 \%){ }^{1} \mathrm{H}$ NMR $\left(300 \mathrm{MHz}\right.$, Methanol- $\left.d_{4}\right) \delta=7.87-7.81(\mathrm{~m}, 2 \mathrm{H}), 7.14(\mathrm{~d}, J=8.1,1 \mathrm{H}), 7.03-6.98(\mathrm{~m}, 2 \mathrm{H}), 6.79-6.77$ $(\mathrm{m}, 1 \mathrm{H}), 6.65-6.60(\mathrm{~m}, 1 \mathrm{H}), 4.54(\mathrm{~d}, J=1.9 \mathrm{~Hz}, 2 \mathrm{H}), 3.87(\mathrm{~s}, 3 \mathrm{H}) .{ }^{13} \mathrm{C} \mathrm{NMR}\left(75 \mathrm{MHz}\right.$, Methanol- $\left.d_{4}\right) \delta=$ $169.74,163.88,149.82,134.80,131.21,130.18,127.64,125.23,116.37,114.71,114.66,55.84,42.09$.

$\mathbf{N}$-(4-Amino-2-chlorobenzyl)-4-(azetidin-1-yl)benzamide (60): Preparation according to general procedure A using 4-(azetidin-1-yl)benzoic acid (51, 0.240 g, 1.35 mmol, 1.00 eq), 1-(4-amino-1chlorophenyl)methanamine (30, $0.319 \mathrm{~g}, 2.03 \mathrm{mmol}, 1.50 \mathrm{eq})$, EDC. $\mathrm{HCl}(0.312 \mathrm{~g}, 1.63 \mathrm{mmol}, 1.20 \mathrm{eq})$ and 4-DMAP $(0.017 \mathrm{~g}, 0.14 \mathrm{mmol}, 0.10 \mathrm{eq})$. The crude product was purified by column chromatography using $\mathrm{n}$-hexane/EtOAc (3:5) as mobile phase to obtain 60 as a yellow oil $(0.111 \mathrm{~g}, 24 \%) .{ }^{1} \mathrm{H}$ NMR (300 $\mathrm{MHz}$, Methanol- $\left.d_{4}\right) \delta=7.75-7.62(\mathrm{~m}, 2 \mathrm{H}), 7.11(\mathrm{~d}, J=8.3 \mathrm{~Hz}, 1 \mathrm{H}), 6.76(\mathrm{t}, J=2.4 \mathrm{~Hz}, 1 \mathrm{H}), 6.69-6.54$ $(\mathrm{m}, 3 \mathrm{H}), 4.52(\mathrm{~d}, J=2.2 \mathrm{~Hz}, 2 \mathrm{H}), 3.74-3.63(\mathrm{~m}, 2 \mathrm{H}), 3.33-3.31(\mathrm{~m}, 2 \mathrm{H}), 2.15-2.00(\mathrm{~m}, 2 \mathrm{H}) .{ }^{13} \mathrm{C} \mathrm{NMR}$ $\left(75 \mathrm{MHz}\right.$, Methanol- $\left.d_{4}\right) \delta=170.40,153.30,149.70,134.73,131.07,130.08,125.66,122.30,116.45$, $114.81,112.39,43.36,41.98,41.10,33.10$.

$\mathbf{N}$-(4-Amino-2-chlorobenzyl)-4-(1-(pyrrolidin-1-yl)benzamide (61): Preparation according to general procedure A using 4-(1-(pyrrolidin-1-yl)benzoic acid (38, 0.200 g, 1.05 mmol, 1.00 eq), 1-(4-amino-1chlorophenyl)methanamine (30, $0.491 \mathrm{~g}, 3.14 \mathrm{mmol}, 3.00 \mathrm{eq}), \mathrm{EDC} \cdot \mathrm{HCl}(0.487 \mathrm{~g}, 3.14 \mathrm{mmol}, 3.00 \mathrm{eq})$ and 4-DMAP $(0.128 \mathrm{~g}, 1.05 \mathrm{mmol}, 1.00 \mathrm{eq})$. The crude product was purified by column chromatography 
using $\mathrm{n}$-hexane/EtOAc (6:1) as mobile phase to obtain 61 as an orange solid $(0.100 \mathrm{~g}, 29 \%) .{ }^{1} \mathrm{H}$ NMR $\left(250 \mathrm{MHz}, \mathrm{DMSO}-d_{6}\right) \delta=8.39(\mathrm{t}, J=5.8 \mathrm{~Hz}, 1 \mathrm{H}), 7.76(\mathrm{~d}, J=8.8 \mathrm{~Hz}, 2 \mathrm{H}), 6.98(\mathrm{~d}, J=8.3 \mathrm{~Hz}, 1 \mathrm{H}), 6.61(\mathrm{~d}$, $J=2.2 \mathrm{~Hz}, 1 \mathrm{H}), 6.53(\mathrm{~d}, J=8.9 \mathrm{~Hz}, 2 \mathrm{H}), 6.47(\mathrm{dd}, J=8.3,2.3 \mathrm{~Hz}, 1 \mathrm{H}), 5.24(\mathrm{~s}, 2 \mathrm{H}), 4.35(\mathrm{~d}, J=5.7 \mathrm{~Hz}, 2 \mathrm{H})$, $3.36-3.24(\mathrm{~m}, 4 \mathrm{H}), 1.96(\mathrm{t}, J=4.8 \mathrm{~Hz}, 4 \mathrm{H}) .{ }^{13} \mathrm{C}$ NMR $\left(75 \mathrm{MHz}\right.$, DMSO- $\left.d_{6}\right) \delta=162.32,149.50,148.89$, $132.23,129.53,128.75,123.11,120.31,113.53,112.59,110.54,47.21,35.78,24.96$.

$\mathbf{N}$-(4-Amino-2-chlorobenzyl)-4-(piperidin-1-yl)benzamide (62): Preparation according to general procedure A using 4-(piperidin-1-yl)benzoic acid (39, $0.200 \mathrm{~g}, 0.974 \mathrm{mmol}, 1.00 \mathrm{eq}), 1$-(4-amino-1chlorophenyl)methanamine (30, $0.458 \mathrm{~g}, 2.92 \mathrm{mmol}, 3.00 \mathrm{eq})$, EDC. $\mathrm{HCl}(0.560 \mathrm{~g}, 2.92 \mathrm{mmol}, 3.00 \mathrm{eq})$ and 4-DMAP $(0.119 \mathrm{~g}, 0.974 \mathrm{mmol}, 1.00 \mathrm{eq})$. The crude product was purified by column chromatography using n-hexane/EtOAc (4:3) as mobile phase to obtain 62 as a yellow solid $(0.133 \mathrm{~g}$, 40\%). ${ }^{1} \mathrm{H}$ NMR $\left(400 \mathrm{MHz}\right.$, DMSO- $\left.d_{6}\right) \delta=8.47(\mathrm{t}, J=5.7 \mathrm{~Hz}, 1 \mathrm{H}), 7.75(\mathrm{~d}, J=8.9 \mathrm{~Hz}, 2 \mathrm{H}), 6.98(\mathrm{~d}, J=8.3$ $\mathrm{Hz}, 1 \mathrm{H}), 6.92(\mathrm{~d}, J=8.9 \mathrm{~Hz}, 2 \mathrm{H}), 6.61(\mathrm{~d}, J=2.3 \mathrm{~Hz}, 1 \mathrm{H}), 6.47(\mathrm{dd}, J=8.3,2.3 \mathrm{~Hz}, 1 \mathrm{H}), 5.24(\mathrm{~s}, 2 \mathrm{H}), 4.34$ $(\mathrm{d}, J=5.6 \mathrm{~Hz}, 2 \mathrm{H}), 3.29-3.26(\mathrm{~m}, 4 \mathrm{H}), 1.58(\mathrm{~s}, 6 \mathrm{H}) .{ }^{13} \mathrm{C} \mathrm{NMR}\left(101 \mathrm{MHz}\right.$, DMSO-d $\left.d_{6}\right) \delta=165.90,152.99$, $148.89,132.23,129.52,128.60,122.92,122.75,113.57,113.52,112.57,48.32,41.27,24.87,23.93$.

$\mathbf{N}$-(4-Amino-2-chlorobenzyl)-4-(morpholin-4-yl)benzamide (63): Preparation according to general procedure A using 4-(morpholin-4-yl)benzoic acid (40, $0.200 \mathrm{~g}, 0.965 \mathrm{mmol}, 1.00 \mathrm{eq}), 1$-(4-amino-1chlorophenyl)methanamine (30, $0.453 \mathrm{~g}, 2.90 \mathrm{mmol}, 3.00 \mathrm{eq}), \mathrm{EDC} \cdot \mathrm{HCl}(0.555 \mathrm{~g}, 2.90 \mathrm{mmol}, 3.00 \mathrm{eq})$ and 4-DMAP $(0.118 \mathrm{~g}, 0.965 \mathrm{mmol}, 1.00 \mathrm{eq})$. The crude product was purified by column chromatography using n-hexane/EtOAc (4:3) as mobile phase to obtain 63 as a yellow solid $(0.107 \mathrm{~g}$, 32\%). ${ }^{1} \mathrm{H}$ NMR $\left(400 \mathrm{MHz}\right.$, DMSO- $\left.d_{6}\right) \delta=8.53(\mathrm{t}, J=5.7 \mathrm{~Hz}, 1 \mathrm{H}), 7.79(\mathrm{~d}, J=8.5 \mathrm{~Hz}, 2 \mathrm{H}), 6.97(\mathrm{~m}, 3 \mathrm{H})$, $6.61(\mathrm{~d}, J=2.3 \mathrm{~Hz}, 1 \mathrm{H}), 6.47(\mathrm{dd}, J=8.3,2.3 \mathrm{~Hz}, 1 \mathrm{H}), 5.25(\mathrm{~s}, 2 \mathrm{H}), 4.35(\mathrm{~d}, J=5.6 \mathrm{~Hz}, 2 \mathrm{H}), 3.73(\mathrm{t}, J=4.8$ $\mathrm{Hz}, 4 \mathrm{H}), 3.21(\mathrm{t}, J=4.8 \mathrm{~Hz}, 4 \mathrm{H}) .{ }^{13} \mathrm{C}$ NMR $\left(101 \mathrm{MHz}, \mathrm{DMSO}-d_{6}\right) \delta=165.85,152.92,148.95,132.30$, $129.61,128.56,124.01,122.86,113.56,113.42,112.61,65.94,47.43,39.86$.

$\mathbf{N}$-(4-Amino-2-chlorobenzyl)-4-(trifluoromethoxy)benzamide (64): Preparation according to general procedure A using 4-(trifluoromethoxy)benzoic acid $(\mathbf{4 1}, 0.350 \mathrm{~g}, 1.70 \mathrm{mmol}, 1.00 \mathrm{eq}), 1$-(4-amino-1chlorophenyl)methanamine (30, $0.346 \mathrm{~g}, 2.21 \mathrm{mmol}, 1.30 \mathrm{eq})$, EDC $\cdot \mathrm{HCl}(0.423 \mathrm{~g}, 2.21 \mathrm{mmol}, 1.30 \mathrm{eq})$ and 4-DMAP $(0.021 \mathrm{~g}, 0.17 \mathrm{mmol}, 0.10 \mathrm{eq})$. The crude product was purified by column chromatography using n-hexane/EtOAc (3:2) as mobile phase to obtain 64 as a yellow oil (0.352 g, 60\%). ${ }^{1} \mathrm{H}$ NMR (300 $\mathrm{MHz}$, Methanol- $\left.d_{4}\right) \delta=7.95-7.90(\mathrm{~m}, 2 \mathrm{H}), 7.36-7.32(\mathrm{~m}, 2 \mathrm{H}), 7.12(\mathrm{~d}, J=8.3 \mathrm{~Hz}, 1 \mathrm{H}), 6.75(\mathrm{~d}, J=2.3$ $\mathrm{Hz}, 1 \mathrm{H}), 6.59$ (dd, $J=8.3,2.3 \mathrm{~Hz}, 1 \mathrm{H}), 4.53(\mathrm{~s}, 2 \mathrm{H}) .{ }^{13} \mathrm{C} \mathrm{NMR}\left(75 \mathrm{MHz}\right.$, Methanol- $\left.d_{4}\right) \delta=168.69$, [152.69, $152.71,152.66,152.64], 150.04,135.00,134.58,131.54,130.56$, [126.89, 123.49, 120.09, 116.69], $124.84,[121.68,121.67,121.66,121.65] 116.42,114.74,42.39$.

$\mathbf{N}$-(4-Amino-2-chlorobenzyl)-4-ethoxybenzamide (65): Preparation according to general procedure $\mathrm{A}$ using 4-ethoxy benzoic acid $\mathbf{( 4 2 ,} 0.400 \mathrm{~g}, 2.41 \mathrm{mmol}, 1.00 \mathrm{eq}), \quad 1$-(4-amino-1chlorophenyl)methanamine (30, $0.490 \mathrm{~g}, 3.13 \mathrm{mmol}, 1.30 \mathrm{eq})$, EDC. $\mathrm{HCl}(0.554 \mathrm{~g}, 2.89 \mathrm{mmol}, 1.20 \mathrm{eq})$ and 4-DMAP $(0.029 \mathrm{~g}, 0.24 \mathrm{mmol}, 0.10 \mathrm{eq})$. The crude product was purified by column chromatography using $\mathrm{n}$-hexane/EtOAc (8:7) as mobile phase to obtain 65 as a beige solid (0.318 g, 43\%). ${ }^{1} \mathrm{H}$ NMR (300 $\mathrm{MHz}$, Methanol- $\left.d_{4}\right) \delta=7.78(\mathrm{~d}, J=8.7 \mathrm{~Hz}, 2 \mathrm{H}), 7.09(\mathrm{~d}, J=8.3 \mathrm{~Hz}, 1 \mathrm{H}), 6.91(\mathrm{~d}, J=8.8 \mathrm{~Hz}, 2 \mathrm{H}), 6.73(\mathrm{~d}$, $J=2.1 \mathrm{~Hz}, 1 \mathrm{H}), 6.57(\mathrm{dd}, J=8.2,2.1 \mathrm{~Hz}, 1 \mathrm{H}), 4.50(\mathrm{~s}, 2 \mathrm{H}), 4.04(\mathrm{q}, J=6.9 \mathrm{~Hz}, 2 \mathrm{H}), 1.37(\mathrm{t}, J=7.0 \mathrm{~Hz}, 3 \mathrm{H})$. ${ }^{13} \mathrm{C}$ NMR $\left(75 \mathrm{MHz}\right.$, Methanol- $\left.d_{4}\right) \delta=169.73,163.16,149.72,134.80,131.22,130.19,127.44,125.29$, $116.43,115.14,114.77,64.68,42.12,15.01$. 
$\mathbf{N}$-(4-Amino-2-chlorobenzyl)-4-isopropyloxybenzamide (66): Preparation according to general procedure A using 4-isopropyloxy benzoic acid $(\mathbf{4 3}, 0.600 \mathrm{~g}, 3.33 \mathrm{mmol}, 1.00 \mathrm{eq}), 1$-(4-amino-1chlorophenyl)methanamine (30, $0.678 \mathrm{~g}, 4.33 \mathrm{mmol}, 1.30 \mathrm{eq})$, EDC. $\mathrm{HCl}(0.766 \mathrm{~g}, 4.00 \mathrm{mmol}, 1.20 \mathrm{eq})$ and 4-DMAP $(0.040 \mathrm{~g}, 0.33 \mathrm{mmol}, 0.10 \mathrm{eq})$. The crude product was purified by column chromatography using $\mathrm{n}$-hexane/EtOAc (3:2) as mobile phase to obtain 66 as a colorless solid $(0.604 \mathrm{~g}, 57 \%) .{ }^{1} \mathrm{H}$ NMR $\left(300 \mathrm{MHz}\right.$, Methanol- $\left.d_{4}\right) \delta=7.82-7.74(\mathrm{~m}, 2 \mathrm{H}), 7.11(\mathrm{~d}, J=8.3 \mathrm{~Hz}, 1 \mathrm{H}), 6.97-6.89(\mathrm{~m}, 2 \mathrm{H}), 6.74(\mathrm{~d}, J$ $=2.3 \mathrm{~Hz}, 1 \mathrm{H}), 6.59(\mathrm{dd}, J=8.3,2.3 \mathrm{~Hz}, 1 \mathrm{H}), 4.67(\mathrm{~h} \mathrm{~J}=6.0 \mathrm{~Hz}, 1 \mathrm{H}), 4.51(\mathrm{~s}, 2 \mathrm{H}), 1.32(\mathrm{~d}, J=6.0 \mathrm{~Hz}, 6 \mathrm{H})$. ${ }^{13} \mathrm{C}$ NMR $\left(75 \mathrm{MHz}\right.$, Methanol- $\left.d_{4}\right) \delta=168.44,160.86,148.49,133.47,129.87,128.88,125.96,123.92$, $115.05,114.87,113.40,69.70,40.75,20.83$.

N-(4-Amino-2-chlorobenzyl)-4-(2-hydroxypropan-2-yl)benzamide (67): Preparation according to general procedure A using 4-(2-hydroxypropan-2-yl)benzoic acid (49, $0.103 \mathrm{~g}, 0.572 \mathrm{mmol}, 1.00 \mathrm{eq})$, 1(4-amino-1-chlorophenyl)methanamine $(30,0.134 \mathrm{~g}, 0.857 \mathrm{mmol}, 1.50 \mathrm{eq}), \mathrm{EDC} \cdot \mathrm{HCl}(0.131 \mathrm{~g}, 0.656$ $\mathrm{mmol}, 1.20 \mathrm{eq})$ and 4-DMAP $(0.007 \mathrm{~g}, 0.06 \mathrm{mmol}, 0.1 \mathrm{eq})$. The crude product was purified by column chromatography using $\mathrm{n}$-hexane/EtOAc (3:5) as mobile phase to obtain 67 as a yellow oil $(0.104 \mathrm{~g}$, 57\%). ${ }^{1} \mathrm{H}$ NMR (400 MHz, Methanol- $\left.d_{4}\right) \delta=7.83-7.77(\mathrm{~m}, 2 \mathrm{H}), 7.59-7.53(\mathrm{~m}, 2 \mathrm{H}), 7.11(\mathrm{~d}, J=8.2 \mathrm{~Hz}$, $1 \mathrm{H}), 6.75-6.74(\mathrm{~m}, 1 \mathrm{H}), 6.60-6.57(\mathrm{~m}, 1 \mathrm{H}), 4.53(\mathrm{~s}, 2 \mathrm{H}), 1.54(\mathrm{~s}, 6 \mathrm{H}) .{ }^{13} \mathrm{C}$ NMR (101 MHz, Methanol$\left.d_{4}\right) \delta=170.04,154.79,149.84,134.87,133.60,131.30,129.32,128.21,125.77,116.45,114.78,72.87$, $42.19,31.76$.

$\mathbf{N}$-(4-Amino-2-chlorobenzyl)-4-(2-methoxypropan-2-yl)benzamide (68): Preparation according to general procedure A using 4-(2-hydroxypropan-2-yl)benzoic acid (50, $0.300 \mathrm{~g}, 1.96 \mathrm{mmol}, 1.00 \mathrm{eq})$, 1(4-amino-1-chlorophenyl)methanamine $(30,0.398 \mathrm{~g}, 2.54 \mathrm{mmol}, 1.30 \mathrm{eq}), \mathrm{EDC} \cdot \mathrm{HCl}(0.488 \mathrm{~g}, 2.54$ $\mathrm{mmol}, 1.30 \mathrm{eq})$ and 4-DMAP $(0.024 \mathrm{~g}, 0.20 \mathrm{mmol}, 0.10 \mathrm{eq})$. The crude product was purified by column chromatography using $\mathrm{n}$-hexane/EtOAc (1:1) as mobile phase to obtain 68 as a colorless solid $(0.352 \mathrm{~g}$, 55\%). ${ }^{1} \mathrm{H}$ NMR (300 MHz, Methanol- $\left.d_{4}\right) \delta=7.86-7.75(\mathrm{~m}, 2 \mathrm{H}), 7.57-7.44(\mathrm{~m}, 2 \mathrm{H}), 7.12(\mathrm{~d}, J=8.4 \mathrm{~Hz}$, $1 \mathrm{H}), 6.75(\mathrm{~d}, J=2.3 \mathrm{~Hz}, 1 \mathrm{H}), 6.59(\mathrm{dd}, J=8.3,2.3 \mathrm{~Hz}, 1 \mathrm{H}), 4.53(\mathrm{~s}, 2 \mathrm{H}), 3.08(\mathrm{~s}, 3 \mathrm{H}), 1.53(\mathrm{~s}, 6 \mathrm{H}) .{ }^{13} \mathrm{C} \mathrm{NMR}$ $\left(75 \mathrm{MHz}\right.$, Methanol- $\left.d_{4}\right) \delta=168.57,149.71,148.58,133.53,132.89,129.97,127.05,125.64,123.72$, $115.05,113.39,76.74,49.60,40.84,26.73$.

$\mathbf{N}$-(4-Amino-2-chlorobenzyl)-4-(1,1-difluoroethyl)benzamide (69): Preparation according to general procedure A using 4-(1,1-difluoroethyl)benzoic acid $(46,0.200 \mathrm{~g}, 1.07 \mathrm{mmol}, 1.00 \mathrm{eq}), 1$-(4-amino-1chlorophenyl)methanamine (30, $0.252 \mathrm{~g}, 1.61 \mathrm{mmol}, 1.50 \mathrm{eq}), \mathrm{EDC} \cdot \mathrm{HCl}(0.245 \mathrm{~g}, 1.29 \mathrm{mmol}, 1.20 \mathrm{eq})$ and 4-DMAP $(0.013 \mathrm{~g}, 0.11 \mathrm{mmol}, 0.10 \mathrm{eq})$. The crude product was purified by column chromatography using $\mathrm{n}$-hexane/EtOAc (5:2) as mobile phase to obtain 69 as a colorless solid $(0.075 \mathrm{~g}, 21 \%) .{ }^{1} \mathrm{H}$ NMR $\left(400 \mathrm{MHz}\right.$, Methanol- $\left.d_{4}\right) \delta=7.90(\mathrm{~d}, J=8.5 \mathrm{~Hz}, 2 \mathrm{H}), 7.61(\mathrm{~d}, J=8.3 \mathrm{~Hz}, 2 \mathrm{H}), 7.13(\mathrm{~d}, J=8.3 \mathrm{~Hz}, 1 \mathrm{H}), 6.75$ $(\mathrm{d}, J=2.3 \mathrm{~Hz}, 1 \mathrm{H}), 6.59(\mathrm{dd}, J=8.3,2.3 \mathrm{~Hz}, 1 \mathrm{H}), 4.54(\mathrm{~s}, 2 \mathrm{H}), 1.92(\mathrm{t}, J=18.3 \mathrm{~Hz}, 3 \mathrm{H}) .{ }^{13} \mathrm{C} \mathrm{NMR}(101 \mathrm{MHz}$, Methanol- $\left.d_{4}\right) \delta=169.33,150.05$, [142.76, 142.50, 142.23], 137.14, 135.00, 131.52, 128.72, [126.00, $125.94,125.88], 124.90,116.42,114.75,42.35$, [26.18, 25.88, 25.59].

N-(4-Amino-2-chlorobenzyl)-4-(2,2,2-trifluoroethyl)benzamide (70): Preparation according to general procedure A using 4-(2,2,2-trifluoroethyl)benzoic acid $(\mathbf{4 7}, 0.285 \mathrm{~g}, 1.40 \mathrm{mmol}, 1.00 \mathrm{eq}), 1$-(4-amino-1chlorophenyl)methanamine (30, $0.328 \mathrm{~g}, 2.09 \mathrm{mmol}, 1.50 \mathrm{eq}), \mathrm{EDC} \cdot \mathrm{HCl}(0.321 \mathrm{~g}, 1.68 \mathrm{mmol}, 1.20 \mathrm{eq})$ and 4-DMAP $(0.017 \mathrm{~g}, 0.14 \mathrm{mmol}, 0.10 \mathrm{eq})$. The crude product was purified by column chromatography using $\mathrm{n}$-hexane/EtOAc (4:3) as mobile phase to obtain 70 as a colorless solid $(0.072 \mathrm{~g}, 15 \%) .{ }^{1} \mathrm{H}$ NMR $\left(400 \mathrm{MHz}\right.$, Methanol- $\left.d_{4}\right) \delta=7.83(\mathrm{~d}, J=8.3 \mathrm{~Hz}, 2 \mathrm{H}), 7.42(\mathrm{~d}, J=8.0 \mathrm{~Hz}, 2 \mathrm{H}), 7.12(\mathrm{~d}, J=8.3 \mathrm{~Hz}, 1 \mathrm{H}), 6.75$ 
(d, $J=2.3 \mathrm{~Hz}, 1 \mathrm{H}$ ), 6.59 (dd, $J=8.3,2.3 \mathrm{~Hz}, 1 \mathrm{H}$ ), $4.53(\mathrm{~s}, 2 \mathrm{H}), 3.54(\mathrm{q}, J=11.1 \mathrm{~Hz}, 2 \mathrm{H}) .{ }^{13} \mathrm{C}$ NMR $(101$ $\mathrm{MHz}$, Methanol- $\left.d_{4}\right) \delta=169.66,149.92$, [135.54, 135.51, 135.48, 135.45], 135.43, 134.93, [131.41, 128.70, 125.96, 123.22], 131.39, 128.65, 125.92, 125.02, 116.44, 114.77, 42.27, [40.72, 40.43, 40.13, 39.84].

$\mathbf{N}$-(4-Amino-2-chlorobenzyl)-4-(pentafluoroethyl)benzamide (71): Preparation according to general procedure A using 4-(pentafluoroethyl) benzoic acid $(48,0.320 \mathrm{~g}, 1.33 \mathrm{mmol}, 1.00 \mathrm{eq}), 1$-(4-amino-1chlorophenyl)methanamine (30, $0.313 \mathrm{~g}, 2.00 \mathrm{mmol}, 1.50 \mathrm{eq}), \mathrm{EDC} \cdot \mathrm{HCl}(0.332 \mathrm{~g}, 1.73 \mathrm{mmol}, 1.30 \mathrm{eq})$ and 4-DMAP $(0.033 \mathrm{~g}, 0.27 \mathrm{mmol}, 0.20 \mathrm{eq})$. The crude product was purified by column chromatography using $\mathrm{n}$-hexane/EtOAc (4:3) as mobile phase to obtain 71 as a colorless solid (0.050 g, 10\%). ${ }^{1} \mathrm{H}$ NMR $\left(500 \mathrm{MHz}\right.$, Methanol- $\left.d_{4}\right) \delta=8.01(\mathrm{~d}, J=8.2 \mathrm{~Hz}, 2 \mathrm{H}), 7.75(\mathrm{~d}, J=8.2 \mathrm{~Hz}, 2 \mathrm{H}), 7.14(\mathrm{~d}, J=8.3 \mathrm{~Hz}, 1 \mathrm{H}), 6.75$ $(\mathrm{d}, J=2.3 \mathrm{~Hz}, 1 \mathrm{H}), 6.60(\mathrm{dd}, J=8.2,2.3 \mathrm{~Hz}, 1 \mathrm{H}), 4.54(\mathrm{~s}, 2 \mathrm{H}) .{ }^{13} \mathrm{C} \mathrm{NMR}\left(126 \mathrm{MHz}\right.$, Methanol- $\left.d_{4}\right) \delta=$ 167.30, 148.75, 133.66, [130.97, 130.78, 130.59], 130.25, 130.25, 127.73, [126.44, 126.39, 126.34], 123.27, $\{[120.48,120.17,119.86],[118.21,117.90,117.59]\},\{[115.73,115.42,115.12,114.81]$, $[113.71,113.41,113.11,112.80],[111.70,111.40,111.09,110.74\}, 115.01,113.34,41.07$.

$\mathbf{N}$-(4-Amino-2-chlorobenzyl)-4-(pentafluorosulfanyl)benzamide (72): Preparation according to general procedure A using 4-(pentafluorosulfanyl)benzoic acid $(\mathbf{4 4}, 0.200 \mathrm{~g}, 0.806 \mathrm{mmol}, 1.00 \mathrm{eq}$ ), 1(4-amino-1-chlorophenyl)methanamine $(30,0.379 \mathrm{~g}, 2.42 \mathrm{mmol}, 3.00 \mathrm{eq}), \mathrm{EDC} \cdot \mathrm{HCl}(0.463 \mathrm{~g}, 2.42$ $\mathrm{mmol}, 3.00 \mathrm{eq})$ and 4-DMAP $(0.098 \mathrm{~g}, 0.806 \mathrm{mmol}, 1.00 \mathrm{eq})$. The crude product was purified by column chromatography using $\mathrm{n}$-hexane/EtOAc (5:3) as mobile phase to obtain $\mathbf{7 2}$ as a colorless solid (0.094 $\mathrm{g}$, 30\%). ${ }^{1} \mathrm{H}$ NMR $\left(300 \mathrm{MHz}\right.$, Acetone- $\left.d_{6}\right) \delta=8.12(\mathrm{~s}, 1 \mathrm{H}), 7.97(\mathrm{~d}, J=8.9 \mathrm{~Hz}, 2 \mathrm{H}), 7.83-7.77(\mathrm{~m}, 2 \mathrm{H}), 7.02$ $(\mathrm{d}, J=8.3 \mathrm{~Hz}, 1 \mathrm{H}), 6.58(\mathrm{~d}, J=2.3 \mathrm{~Hz}, 1 \mathrm{H}), 6.44(\mathrm{dd}, J=8.3,2.3 \mathrm{~Hz}, 1 \mathrm{H}), 4.75(\mathrm{~s}, 2 \mathrm{H}), 4.41(\mathrm{~d}, J=5.6 \mathrm{~Hz}$, $2 \mathrm{H}) .{ }^{13} \mathrm{C}$ NMR $\left(75 \mathrm{MHz}\right.$, Acetone- $\left.d_{6}\right) \delta=165.72$, [156.18, 155.96, 155.74], 150.11, 139.16, 134.46, 131.68, 129.07, [127.03, 126.97, 126.90, 126.84, 126.78], 123.91, 115.24, 113.88, 41.89.

tert-Butyl-(3,5-dichloro-4-methylphenyl)carbamate (89): Di-tert-butyl dicarbonate $(1.00 \mathrm{~g}, 4.58$ $\mathrm{mmol}, 1.00 \mathrm{eq})$ and 3,5-dichloro-4-methylaniline (88, $0.968 \mathrm{~g}, 5.50 \mathrm{mmol}, 1.20 \mathrm{eq})$ were dissolved in THF $(30 \mathrm{~mL})$. The resulting mixture was stirred at $60^{\circ} \mathrm{C}$ for $16 \mathrm{~h} . \mathrm{H}_{2} \mathrm{O}(30 \mathrm{~mL})$ and EtOAc $(5 \mathrm{~mL})$ were added, the phases were separated and the aqueous layer was extracted with EtOAc $(3 \times 30 \mathrm{~mL})$. The combined organic layers were dried over $\mathrm{Na}_{2} \mathrm{SO}_{4}$ and the solvent was removed in vacuum. Further purification was performed by column chromatography using hexane/EtOAc $(5: 1)$ as mobile phase to obtain 89 as a brown solid (1.05 g, 83\%). ${ }^{1} \mathrm{H}$ NMR (400 MHz, Chloroform-d) $\delta=7.34$ (s, 2H), 2.37 (s, $3 \mathrm{H}), 1.51$ (s, 9H). ${ }^{13} \mathrm{C}$ NMR (101 MHz, Chloroform-d) $\delta=152.45,137.25,135.57,128.64,117.78,81.37$, $28.38,16.82$.

tert-Butyl (4-(bromomethyl)-3,5-dichlorophenyl)carbamate (90): tert-Butyl (3,5-dichloro-4methylphenyl)carbamate $(89,0.800 \mathrm{~g}, 2.90 \mathrm{mmol}, 1.00 \mathrm{eq})$ and $\mathrm{N}$-bromosuccinimide $(0.773 \mathrm{~g}, 4.35$ $\mathrm{mmol}, 1.50 \mathrm{eq})$ were dissolved in chloroform $(20 \mathrm{~mL})$. Azobisisobutyronitrile $(0.048 \mathrm{~g}, 0.290 \mathrm{mmol}$, $0.100 \mathrm{eq}$ ) was added carefully under stirring at room temperature before the resulting mixture was stirred at $60^{\circ} \mathrm{C}$ for $3 \mathrm{~h}$. After cooling to room temperature, $\mathrm{H}_{2} \mathrm{O}(2 \mathrm{~mL})$ was added, the phases were separated and the aqueous layer was extracted with EtOAc $(3 \times 30 \mathrm{~mL})$. The combined organic layers were dried over $\mathrm{Na}_{2} \mathrm{SO}_{4}$ and the solvent was removed in vacuum. Further purification was performed by column chromatography using hexane/EtOAc (5:1) to obtain 90 as a yellow solid $(0.793 \mathrm{~g}, 77 \%){ }^{1} \mathrm{H}$ NMR (500 MHz, DMSO- $\left.d_{6}\right) \delta=9.86(\mathrm{~s}, 1 \mathrm{H}) 7.59(\mathrm{~s}, 2 \mathrm{H}), 4.74(\mathrm{~s}, 2 \mathrm{H}), 1.47(\mathrm{~s}, 9 \mathrm{H}) .{ }^{13} \mathrm{C} N M R(126 \mathrm{MHz}$, DMSO- $\left.d_{6}\right) \delta=152.52,141.68,135.44,135.18,117.33,80.22,28.04,28.00$. 
tert-Butyl (4-(aminomethyl)-3,5-dichlorophenyl)carbamate (91): tert-Butyl (4-(bromomethyl)-3,5dichlorophenyl)carbamate (90, $0.370 \mathrm{~g}, 1.04 \mathrm{mmol}, 1.00 \mathrm{eq})$ was dissolved in DMF (20 mL), $\mathrm{NaN}_{3}(0.102$ $\mathrm{g}, 1.56 \mathrm{mmol}, 1.50 \mathrm{eq}$ ) was added and the mixture was stirred at $80^{\circ} \mathrm{C}$ for $12 \mathrm{~h}$. After cooling to room temperature and addition of brine $(20 \mathrm{~mL})$, the aqueous layer was extracted with EtOAc $(3 \times 50 \mathrm{~mL})$. The combined organic layers were dried over $\mathrm{Na}_{2} \mathrm{SO}_{4}$ and the solvent was removed in vacuum. The resulting azide was dissolved in a mixture of $\mathrm{H}_{2} \mathrm{O}(4 \mathrm{~mL})$ and THF $(30 \mathrm{~mL}) . \mathrm{PPh}_{3}(0.327 \mathrm{~g}, 1.25 \mathrm{mmol}$, $1.20 \mathrm{eq}$ ) was added and the mixture was stirred at room temperature for $17 \mathrm{~h}$. Afterwards, the mixture was diluted with $\mathrm{H}_{2} \mathrm{O}(30 \mathrm{~mL})$, adjusted to an alkaline $\mathrm{pH}$ with $\mathrm{Na}_{2} \mathrm{CO}_{3}$ and extracted with EtOAc $(3 \times 50 \mathrm{~mL})$. The combined organic layers were dried over $\mathrm{Na}_{2} \mathrm{SO}_{4}$ and the solvent was removed in vacuum. The crude product was dissolved in EtOAc $(20 \mathrm{~mL})$ and a solution of $\mathrm{HCl}$ in dioxane $(4 \mathrm{M}, 1$ $\mathrm{mL}$ ) was added to precipitate the hydrochloride 91 as a pale yellow solid (0.080 g, $24 \%$ ). ${ }^{1} \mathrm{H}$ NMR (500 $\left.\mathrm{MHz}, \mathrm{DMSO}-d_{6}\right) \delta=9.70(\mathrm{~s}, 1 \mathrm{H}), 7.55(\mathrm{~s}, 2 \mathrm{H}), 4.43(\mathrm{~s}, 2 \mathrm{H}), 1.94(\mathrm{~s}, 3 \mathrm{H}), 1.48(\mathrm{~s}, 9 \mathrm{H}) .{ }^{13} \mathrm{C} \mathrm{NMR}(126 \mathrm{MHz}$, DMSO- $\left.d_{6}\right) \delta=152.47,140.02,135.12,129.20,117.12,80.06,50.29,27.99$.

$\mathbf{N}$-(4-Amino-2,6-dichlorobenzyl)-4-(tert-butyl)benzamide (92): 4-(tert-Butyl)benzoic acid (33, $0.080 \mathrm{~g}$, $0.45 \mathrm{mmol}, 1.0 \mathrm{eq}$ ), tert-butyl (4-(aminomethyl)-3,5-dichlorophenyl)carbamate $(\mathbf{9 1}, 0.13 \mathrm{~g}, 0.45 \mathrm{mmol}$, $1.0 \mathrm{eq})$ and 4-DMAP $(0.005 \mathrm{~g}, 0.05 \mathrm{mmol}, 0.1 \mathrm{eq})$ were dissolved in a mixture of chloroform $(7.5 \mathrm{~mL})$ and DMF $(7.5 \mathrm{~mL})$. After cooling to $0^{\circ} \mathrm{C}$ EDC. $\mathrm{HCl}(0.10 \mathrm{~g}, 0.54 \mathrm{mmol}, 1.2 \mathrm{eq})$ was added. The reaction mixture was stirred at $0^{\circ} \mathrm{C}$ for 30 minutes was refluxed for $16 \mathrm{~h}$. Aqueous hydrochloric acid $(10 \%, 15$ $\mathrm{mL}$ ) was added and the phases were separated. The aqueous layer was brought to $\mathrm{pH} 12$ using $\mathrm{Na}_{2} \mathrm{CO}_{3}$ and extracted with EtOAc $(3 \times 20 \mathrm{~mL})$. The combined organic layers were dried over $\mathrm{Na}_{2} \mathrm{SO}_{4}$ and the solvent was removed in vacuum yielding the intermediate tert-butyl (4-(44-(tertbutyl)benzamido)methyl)-3,5-dichlorophenyl)carbamate (MS (ESI+) $m / z 473.01\left([\mathrm{M}+\mathrm{Na}]^{+}\right)$) which was dissolved in a mixture of methylene chloride $(5 \mathrm{~mL})$ and trifluoroacetic acid $(1 \mathrm{~mL})$. The resulting mixture was stirred at room temperature for $2 \mathrm{~h}$ and then quenched by the addition of a saturated aqueous solution of $\mathrm{NaHCO}_{3}(10 \mathrm{~mL})$. The phases were separated and the aqueous layer was extracted with EtOAc $(3 \times 20 \mathrm{~mL})$. The combined organic layers were dried over $\mathrm{Na}_{2} \mathrm{SO}_{4}$ and the solvent was removed in vacuum. Further purification was performed by column chromatography using $\mathrm{n}$ hexane/EtOAc (5:1) as mobile phase to obtain 92 as a yellow solid. (0.077 g, 49\% over two steps). ${ }^{1} \mathrm{H}$ NMR $\left(400 \mathrm{MHz}\right.$, Methanol- $\left.d_{4}\right) \delta=7.76-7.68(\mathrm{~m}, 2 \mathrm{H}), 7.49-7.42(\mathrm{~m}, 4 \mathrm{H}), 6.69(\mathrm{~s}, 2 \mathrm{H}), 4.70(\mathrm{~s}, 2 \mathrm{H})$, $1.32(s, 9 H) .{ }^{13} \mathrm{C}$ NMR $\left(101 \mathrm{MHz}\right.$, Methanol- $\left.d_{4}\right) \delta=170.18,156.13,151.06,137.75,132.71,128.34$, $126.35,121.07,114.98,40.57,35.72,31.55$.

4-(3-Hydroxyoxetan-3-yl)benzoic acid (95): 4-Bromobenzoic acid (93, 0.400 g, $1.99 \mathrm{mmol}, 1.00 \mathrm{eq}$ ) was dissolved in THF $(9 \mathrm{~mL})$ and cooled to $-78^{\circ} \mathrm{C}$. A $15 \%$ solution of $n$-butyllithium $(0.318 \mathrm{~g}, 4.97 \mathrm{mmol}, 2.50$ eq) in hexane was added dropwise and the mixture was stirred for 30 minutes at -78. 3-oxetanone (94, $0.215 \mathrm{~g}, 2.98 \mathrm{mmol}, 1.50 \mathrm{eq}$ ) was added carefully, the mixture was stirred for 60 minutes at $-78^{\circ} \mathrm{C}$, then allowed to warm up to room temperature and diluted with EtOAc $(10 \mathrm{~mL})$. Aqueous hydrochloric acid $(10 \%, 15 \mathrm{~mL})$ was added and the phases were separated, and the aqueous layer was extracted with ethyl acetate $(3 \times 15 \mathrm{~mL})$. The combined organic layers were dried over $\mathrm{Na}_{2} \mathrm{SO}_{4}$ and the solvent was removed in vacuum. Further purification was performed by column chromatography using hexane/EtOAc (1:2) + 2\% acetic acid as mobile phase to obtain 95 as a colorless solid $(0.185 \mathrm{~g}, 48 \%)$. ${ }^{1} \mathrm{H}$ NMR $\left(250 \mathrm{MHz}\right.$, DMSO- $\left.d_{6}\right) \delta=8.04-7.92(\mathrm{~m}, 2 \mathrm{H}), 7.77-7.68(\mathrm{~m}, 2 \mathrm{H}), 6.50(\mathrm{~s}, 1 \mathrm{H}), 4.79(\mathrm{~d}, J=6.8$ 
$\mathrm{Hz}, 2 \mathrm{H}), 4.67(\mathrm{~d}, J=6.8 \mathrm{~Hz}, 2 \mathrm{H}) .{ }^{13} \mathrm{C} \mathrm{NMR}\left(75 \mathrm{MHz}, \mathrm{DMSO}-d_{6}\right) \delta=159.87,149.46,129.84,125.16,124.79$, $85.63,80.42$.

4-(3-Acetoxyoxetan-3-yl)benzoic acid (96): 4-(3-Hydroxyoxetan-3-yl)benzoic acid (95, 0.200 g, 1.03 $\mathrm{mmol}, 1.00 \mathrm{eq})$ was dissolved in a mixture of methylene chloride $(5 \mathrm{~mL})$ and triethylamine $(0.5 \mathrm{~mL})$ and stirred at room temperature for 10 minutes. After a dropwise addition of acetylchloride $(0.162 \mathrm{~g}, 2.06$ $\mathrm{mmol}, 2.00 \mathrm{eq})$, the mixture was stirred under reflux for $16 \mathrm{~h}$. Aqueous hydrochloric acid $(10 \%, 5 \mathrm{~mL})$ was added, phases were separated and the aqueous layer was extracted with EtOAc ( $3 \times 15 \mathrm{~mL})$. The combined organic layers were dried over $\mathrm{Na}_{2} \mathrm{SO}_{4}$ and the solvent was evaporated vacuum. Further purification was performed by column chromatography using $n$-hexane/EtOAc/acetic acid (65:32:2) as mobile phase to yield 96 as a colorless solid $(0.170 \mathrm{~g}, 70 \%) .{ }^{1} \mathrm{H}$ NMR $\left(250 \mathrm{MHz}\right.$, Methanol- $\left.d_{4}\right) \delta=8.05$ $-7.99(\mathrm{~m}, 2 \mathrm{H}), 7.68-7.64(\mathrm{~m}, 2 \mathrm{H}), 4.46-4.33(\mathrm{~m}, 2 \mathrm{H}), 4.01-3.88(\mathrm{~m}, 2 \mathrm{H}), 2.01(\mathrm{~s}, 3 \mathrm{H}) .{ }^{13} \mathrm{C} \mathrm{NMR}(101$ $\mathrm{MHz}$, Methanol- $\left.d_{4}\right) \delta=172.24,169.56,147.87,131.35,130.58,127.29,76.08,69.37,20.58$.

3-(4-((4-Amino-2-chlorobenzyl)carbamoyl)phenyl)oxetan-3-yl acetate (97): Preparation according to general procedure A using 4-(3-acetoxyoxetan-3-yl)benzoic acid (96, 0.170 g, $0.720 \mathrm{mmol}, 1.00 \mathrm{eq}), 1$ (4-amino-1-chlorophenyl)methanamine (30, $0.169 \mathrm{~g}, 1.08 \mathrm{mmol}, 1.50 \mathrm{eq})$, 4-DMAP (0.009 g, 0.07 $\mathrm{mmol}, 0.1 \mathrm{eq})$ and EDC. $\mathrm{HCl}(0.166 \mathrm{~g}, 0.864 \mathrm{mmol}, 1.20 \mathrm{eq})$. The crude product was purified by column chromatography using $\mathrm{n}$-hexane/EtOAc (4:3) as mobile phase to obtain 97 as a yellow oil $(0.132 \mathrm{~g}$, 49\%). ${ }^{1} \mathrm{H}$ NMR $\left(500 \mathrm{MHz}\right.$, Methanol- $\left.d_{4}\right) \delta=7.86-7.82(\mathrm{~m}, 2 \mathrm{H}), 7.65-7.60(\mathrm{~m}, 2 \mathrm{H}), 7.12(\mathrm{~d}, J=8.3 \mathrm{~Hz}$, $1 \mathrm{H}), 6.75(\mathrm{~d}, J=2.3 \mathrm{~Hz}, 1 \mathrm{H}), 6.60(\mathrm{dd}, J=8.3,2.3 \mathrm{~Hz}, 1 \mathrm{H}), 4.53(\mathrm{~s}, 2 \mathrm{H}), 4.44-4.33(\mathrm{~m}, 2 \mathrm{H}), 3.98-3.91$ $(\mathrm{m}, 2 \mathrm{H}), 2.00(\mathrm{~s}, 3 \mathrm{H}) .{ }^{13} \mathrm{C}$ NMR $\left(75 \mathrm{MHz}\right.$, Methanol- $\left.d_{4}\right) \delta=172.26,169.83,149.94,146.38,135.02$, $134.91,131.37,128.27,127.34,125.07,116.45,114.79,76.00,69.38,42.25,20.76$.

3-(4-((2-Chloro-4-(methylsulfonamido)benzyl)carbamoyl)phenyl)oxetan-3-yl acetate (98): Preparation according to general procedure B using 3-(4-((4-amino-2chlorobenzyl)carbamoyl)phenyl)oxetan-3-yl acetate (97, $0.065 \mathrm{~g}, 0.17 \mathrm{mmol}, 1.0 \mathrm{eq})$ and methanesulfonyl chloride $(73,0.20 \mathrm{~g}, 1.7 \mathrm{mmol}, 10 \mathrm{eq})$. The crude product was purified by column chromatography using $n$-hexane/EtOAc (3:2) as mobile phase to obtain 98 as a colorless solid $(0.044 \mathrm{~g}$, $55 \%) .{ }^{1} \mathrm{H}$ NMR $\left(300 \mathrm{MHz}, \mathrm{DMSO}-d_{6}\right) \delta=8.98(\mathrm{t}, J=5.8 \mathrm{~Hz}, 1 \mathrm{H}), 7.92-7.85(\mathrm{~m}, 2 \mathrm{H}), 7.64-7.60(\mathrm{~m}, 2 \mathrm{H})$, $7.32(\mathrm{~d}, J=8.4 \mathrm{~Hz}, 1 \mathrm{H}), 7.26(\mathrm{~d}, J=2.2 \mathrm{~Hz}, 1 \mathrm{H}), 7.14(\mathrm{dd}, J=8.4,2.2 \mathrm{~Hz}, 1 \mathrm{H}), 4.49(\mathrm{~d}, J=5.8 \mathrm{~Hz}, 2 \mathrm{H}), 4.34$ - $4.22(\mathrm{~m}, 2 \mathrm{H}), 4.02-3.92(\mathrm{~m}, 2 \mathrm{H}), 3.00(\mathrm{~s}, 3 \mathrm{H}), 1.97(\mathrm{~s}, 3 \mathrm{H}) .{ }^{13} \mathrm{C}$ NMR $\left(75 \mathrm{MHz}, \mathrm{DMSO}-d_{6}\right) \delta=170.04$, $166.20,145.04,138.86,133.14,132.33,131.25,129.61,126.96,126.03,119.73,118.31,74.29,68.00$, $50.49,39.42,20.64$. 


\section{HPLC traces of 4-29}

4-(tert-butyl)-N-(2-chloro-4-(methylsulfonamido)benzyl)benzamide (3)<smiles>CC(C)(C)c1ccc(C(=O)NCc2ccc(NS(C)(=O)=O)cc2Cl)cc1</smiles>

\section{$254 \mathrm{~nm}$}

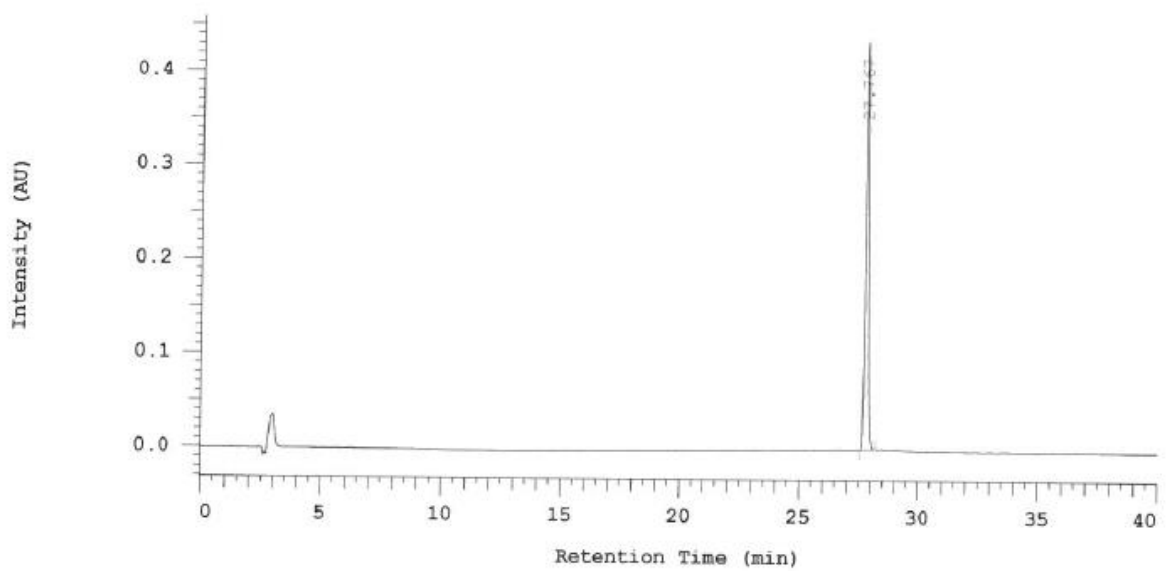

Pump 1: 5160

Pump 1 Solvent A: $\mathrm{MeOH}$

Pump 1 Solvent C: $\mathrm{MeOH}$

Pump 1 Solvent B: Wasser

Pump 1 Solvent D: $\mathrm{MeOH}$

\begin{tabular}{ccccc} 
No. & RT & Area & Area $\%$ & Height \\
\hline 1 & 27.767 & 1964272 & 100.000 & 216627 \\
\hline & 1964272 & 100.000 & 216627 \\
\hline
\end{tabular}

\section{$280 \mathrm{~nm}$}

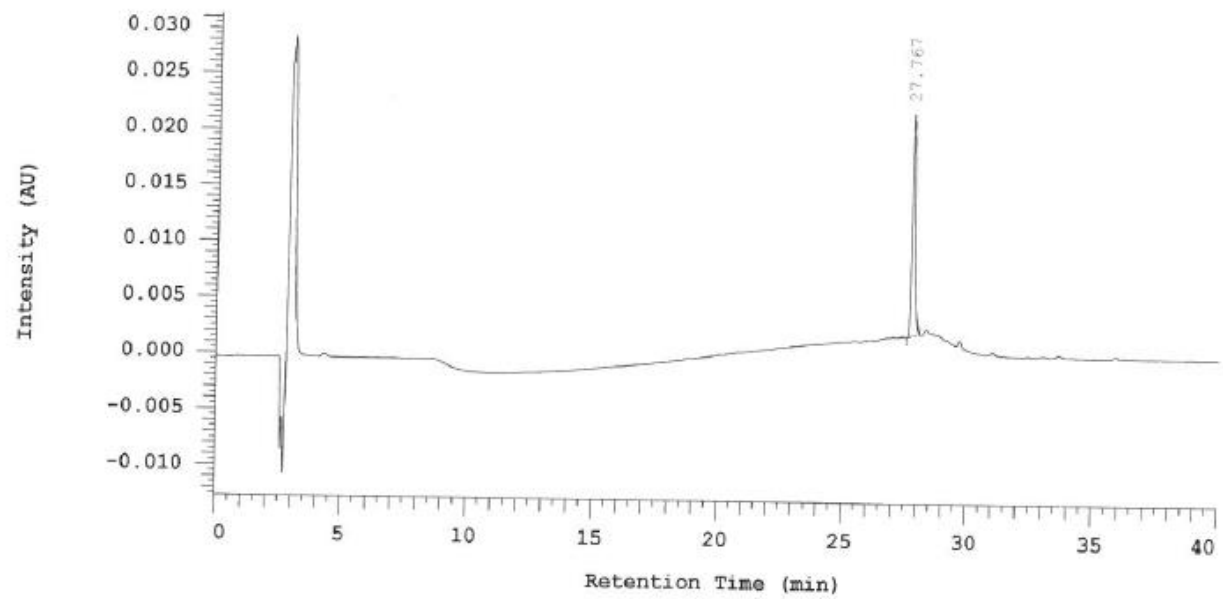

Pump 1: 5160

Pump 1 Solvent A: $\mathrm{MeOH}$

Pump 1 Solvent C: $\mathrm{MeOH}$

Pump 1 Solvent B: Wasser

Pump 1 Solvent D: $\mathrm{MeOH}$

\begin{tabular}{ccccr} 
No. & RT & Area & Area \& & Height \\
\hline 1 & 27.767 & 90683 & 100.000 & 9940 \\
\hline & 90683 & 100.000 & 9940 \\
\hline
\end{tabular}


4-(tert-Butyl)-N-(2-chloro-4-(ethylsulfonamido)benzyl)benzamide (4)<smiles>CCS(=O)(=O)Nc1ccc(CNC(=O)c2ccc(C(C)(C)C)cc2)c(Cl)c1</smiles>

\section{$254 \mathrm{~nm}$}

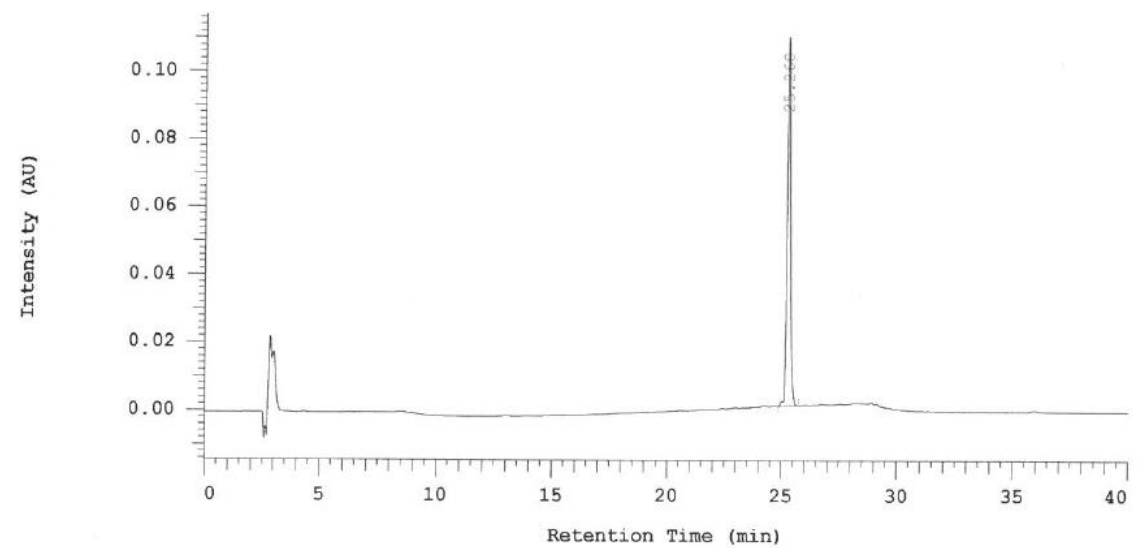

Pump 1: 5160

Pump 1 Solvent A: $\mathrm{MeOH}$

Pump 1 Solvent C: $\mathrm{MeOH}$

Pump 1 Solvent B: Wasser

Pump 1 Solvent D: $\mathrm{MeOH}$

\begin{tabular}{ccccr} 
No. & RT & Area & Area : & Height \\
\hline 1 & 25.260 & 548425 & 100.000 & 54429 \\
\hline & & 548425 & 100.000 & 54429 \\
\hline
\end{tabular}

\section{$280 \mathrm{~nm}$}

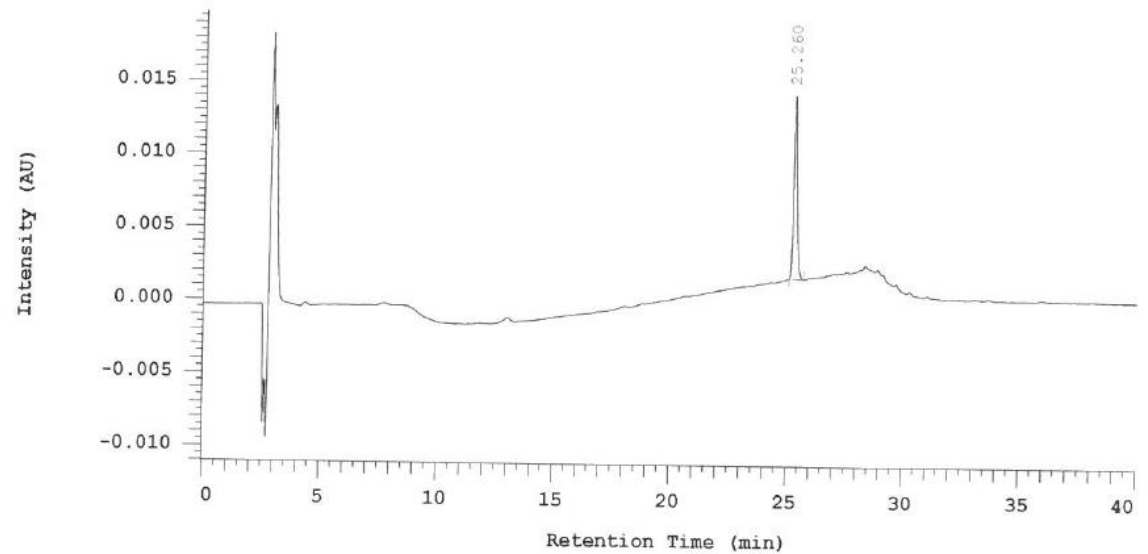

Pump 1: 5160

Pump 1 Solvent A: $\mathrm{MeOH}$

Pump 1 Solvent C: $\mathrm{MeOH}$

Pump 1 Solvent B: Wasser

Pump 1 Solvent D: $\mathrm{MeOH}$

\begin{tabular}{ccccr} 
No. & RT & Area & Area \& & Height \\
\hline 1 & 25.260 & 62842 & 100.000 & 6283 \\
\hline & 62842 & 100.000 & 6283 \\
\hline
\end{tabular}


4-(tert-Butyl)-N-(2-chloro-4-(isopropylsulfonamido)benzyl)benzamide (5)<smiles>CC(C)S(=O)(=O)Nc1ccc(CNC(=O)c2ccc(C(C)(C)C)cc2)c(Cl)c1</smiles>

\section{$254 \mathrm{~nm}$}

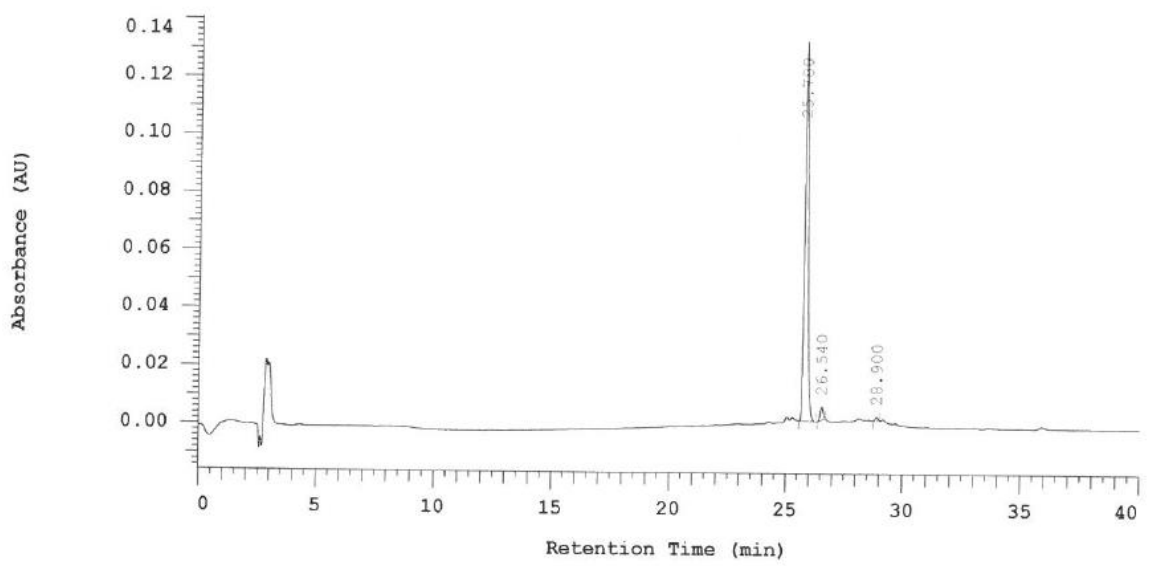

Pump 1: 5160

Pump 1 Solvent A: $\mathrm{MeOH}$

Pump 1 Solvent $\mathrm{C}$ : $\mathrm{MeOH}$

Pump 1 Solvent B: Wasser

\begin{tabular}{rrrrr} 
No. & RT & Area & Area \% & Height \\
\hline 1 & 25.780 & 676129 & 96.444 & 65458 \\
2 & 26.540 & 19254 & 2.746 & 2279 \\
3 & 28.900 & 5673 & 0.809 & 686 \\
\hline & & 701056 & 100.000 & 68423 \\
\hline
\end{tabular}

\section{$280 \mathrm{~nm}$}

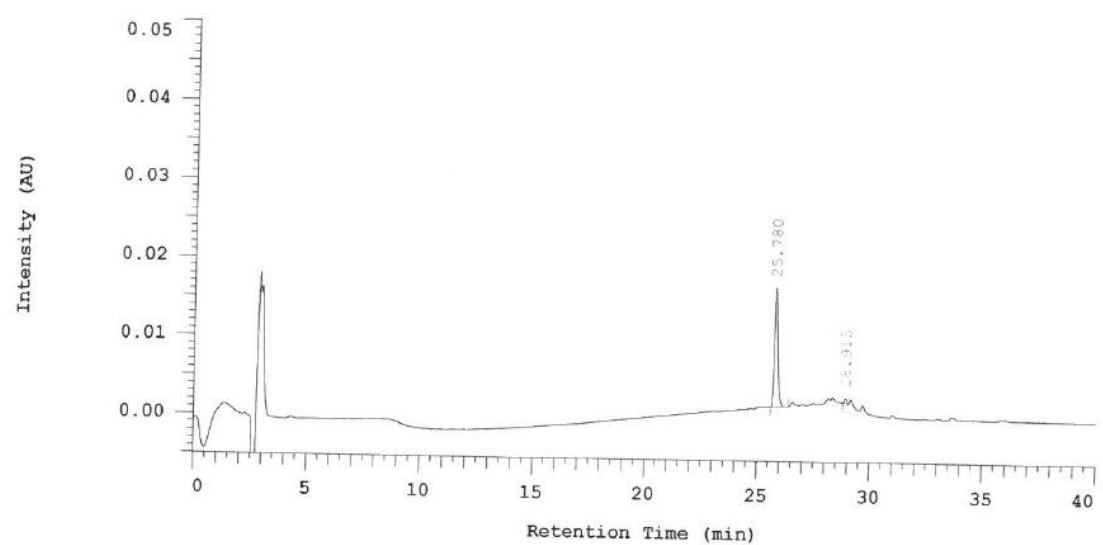

Pump 1: 5160

Pump 1 Solvent A: $\mathrm{MeOH}$

Pump 1 Solvent C: $\mathrm{MeOH}$

Pump 1 Solvent $B$ : Wasser

\begin{tabular}{rrrrr} 
No. & RT & Area & Area \& & Height \\
\hline 1 & 25.780 & 78855 & 97.000 & 7593 \\
2 & 28.913 & 2439 & 3.000 & 325 \\
\hline & & 81294 & 100.000 & 7918 \\
\hline
\end{tabular}


4-(tert-Butyl)-N-(2-chloro-4-(cyclopropylsulfonamido)benzyl)benzamide (6)<smiles>CC(C)(C)c1ccc(C(=O)NCc2ccc(NS(=O)(=O)C3CC3)cc2Cl)cc1</smiles>

$254 \mathrm{~nm}$

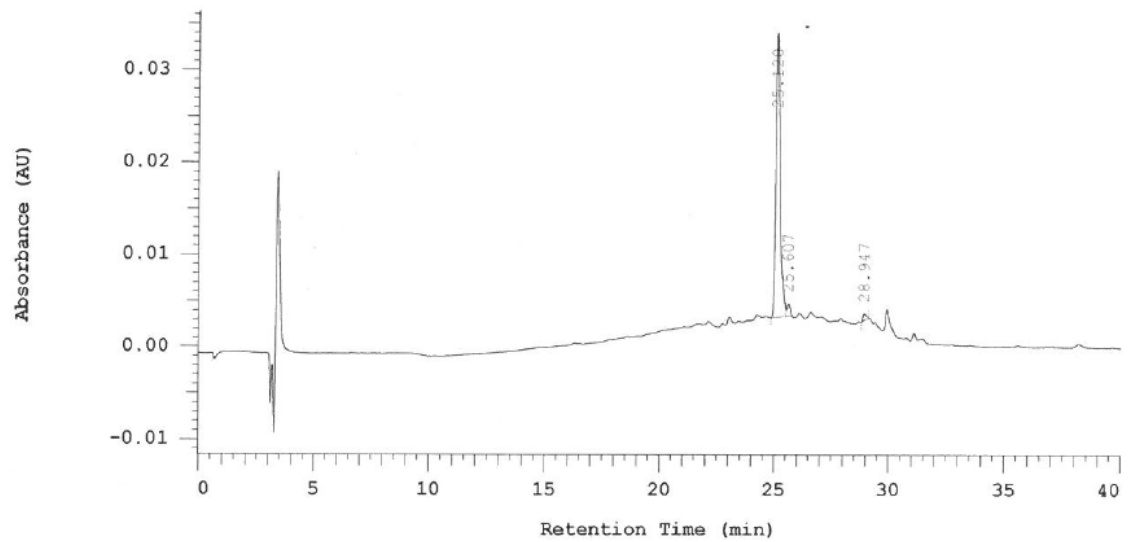

Pump 1: 5160

Pump 1 Solvent A: MeOH Pump 1 Solvent B: Wasser

Pump 1 Solvent C: $\mathrm{MeOH} \quad$ Pump 1 Solvent D: MeOH

\begin{tabular}{rrrrr} 
No. & RT & Area & Area \% & Height \\
\hline 1 & 25.120 & 179094 & 95.091 & 15361 \\
2 & 25.607 & 6145 & 3.263 & 643 \\
3 & 28.947 & 3099 & 1.646 & 360 \\
\hline & & 188338 & 100.000 & 16364
\end{tabular}

$280 \mathrm{~nm}$

-no absorbance @280 nm- 
<smiles>CC(C)(C)c1ccc(C(=O)NCc2ccc(NS(=O)(=O)C(F)(F)F)cc2Cl)cc1</smiles>

\section{$254 \mathrm{~nm}$}

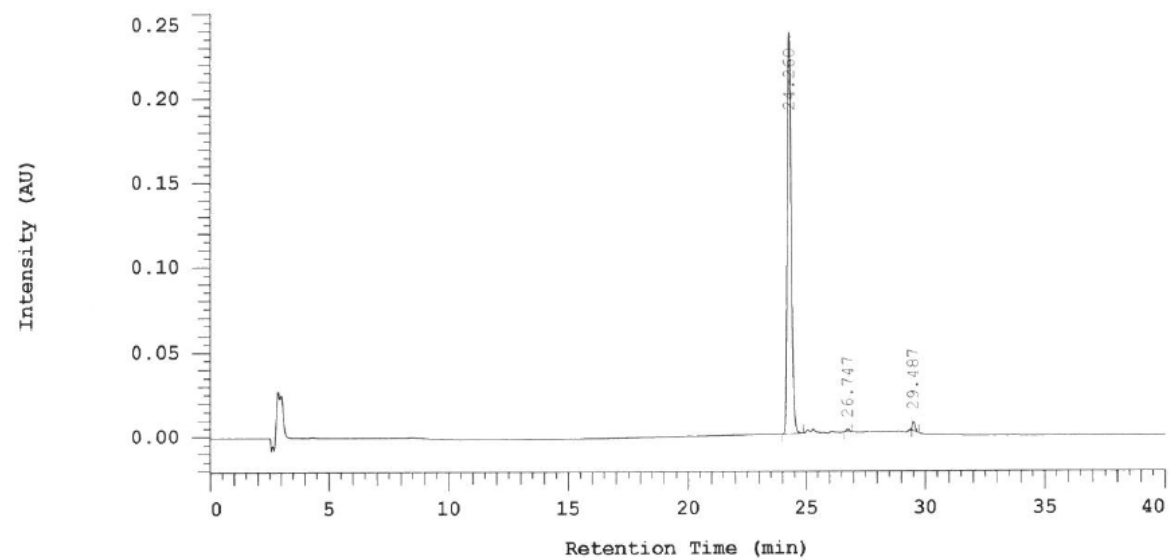

Pump 1: 5160

Pump 1 Solvent A: MeOH Pump 1 Solvent B: Wasser

Pump 1 Solvent $\mathrm{C}: \mathrm{MeOH}$

$$
\text { Pump } 1 \text { Solvent D: } \mathrm{MeOH}
$$

\begin{tabular}{rrrrr} 
No. & RT & \multicolumn{1}{c}{ Area } & Area & Height \\
\hline 1 & 24.260 & 1252611 & 97.942 & 118376 \\
2 & 26.747 & 5914 & 0.462 & 676 \\
3 & 29.487 & 20407 & 1.596 & 2631 \\
\hline & 1278932 & 100.000 & 121683
\end{tabular}

\section{$280 \mathrm{~nm}$}

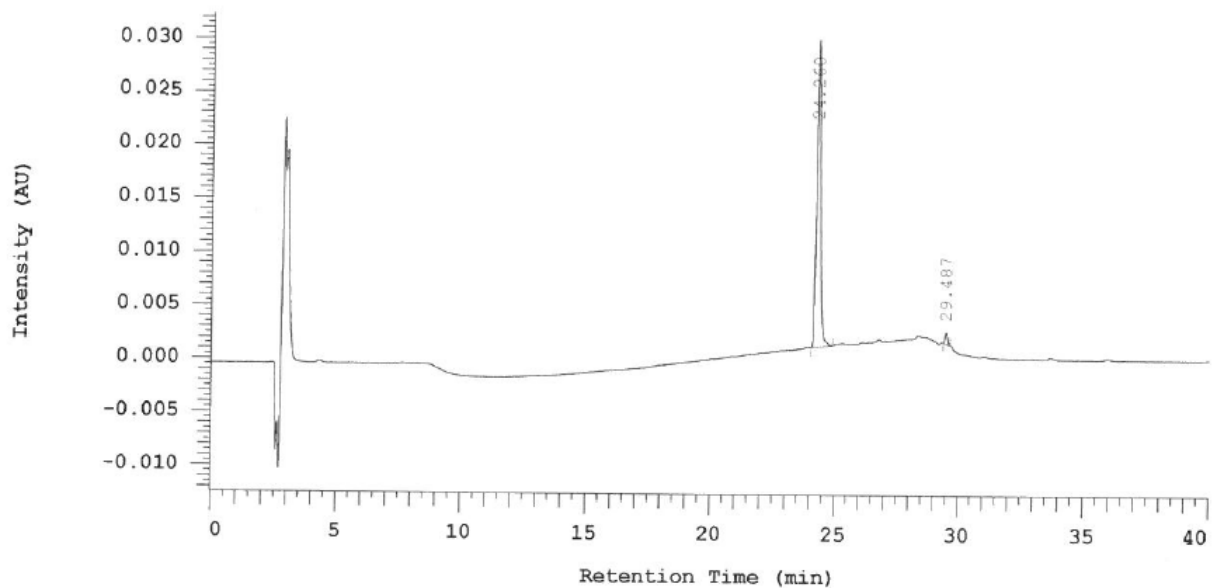

Pump 1: 5160

Pump 1 Solvent $A$ : $\mathrm{MeOH}$

Pump 1 Solvent $\mathrm{C}$ : $\mathrm{MeOH}$

Pump 1 Solvent B: Wasser

Pump 1 Solvent D: $\mathrm{MeOH}$

\begin{tabular}{rrrrr} 
No. & RT & Area & Area & Height \\
\hline 1 & 24.260 & 154663 & 97.729 & 14406 \\
2 & 29.487 & 3594 & 2.271 & 508 \\
\hline & & 158257 & 100.000 & 14914 \\
\hline
\end{tabular}


4-(tert-Butyl)- $N$-(2-fluoro-4-(methylsulfonamido)benzyl)benzamide (8)<smiles>CC(C)(C)c1ccc(C(=O)NCc2ccc(NS(C)(=O)=O)cc2F)cc1</smiles>

\section{$254 \mathrm{~nm}$}

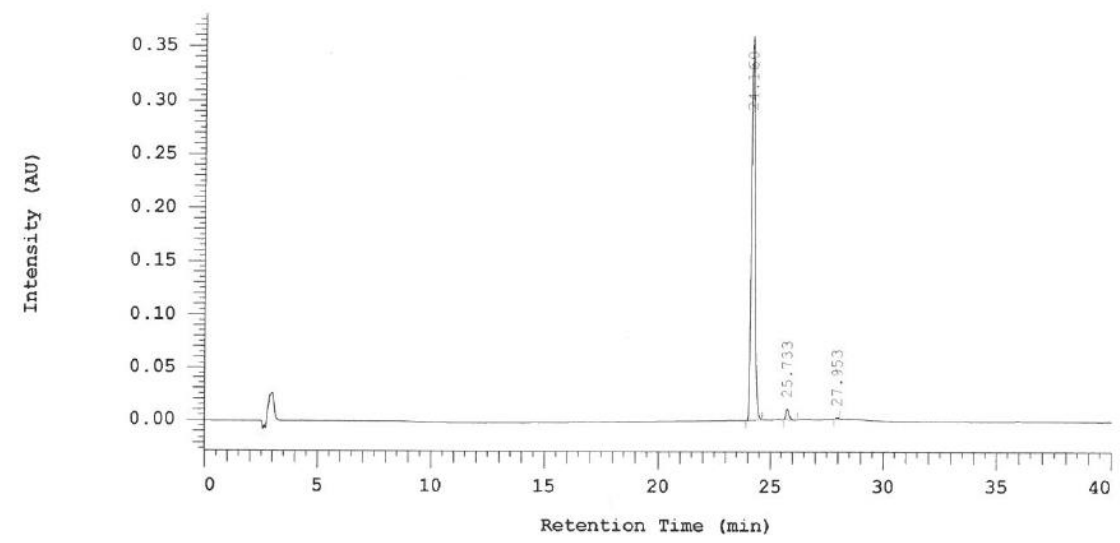

Pump 1: 5160

Pump 1 Solvent A: MeOH Pump 1 Solvent B: Wasser

Pump 1 Solvent $\mathrm{C}$ : $\mathrm{MeOH} \quad$ Pump 1 Solvent D: MeOH

\begin{tabular}{rrrrr} 
No. & RT & \multicolumn{1}{c}{ Area } & Area & Height \\
\hline 1 & 24.160 & 1863278 & 97.234 & 179542 \\
2 & 25.733 & 48154 & 2.513 & 5002 \\
3 & 27.953 & 4842 & 0.253 & 625 \\
\hline & & 1916274 & 100.000 & 185169 \\
\hline
\end{tabular}

\section{$280 \mathrm{~nm}$}

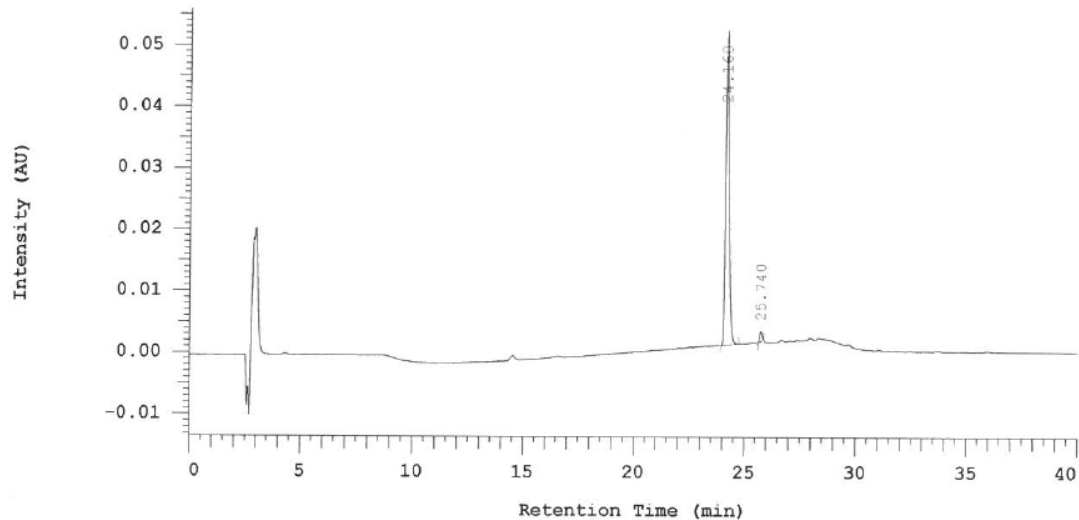

Pump 1: 5160

Pump 1 Solvent A: $\mathrm{MeOH}$

Pump 1 Solvent $\mathrm{C}$ : $\mathrm{MeOH}$

Pump 1 Solvent B: Wasser

\begin{tabular}{rrrrr} 
No. & RT & Area & Area \% & Height \\
\hline 1 & 24.160 & 268469 & 97.841 & 25573 \\
2 & 25.740 & 5924 & 2.159 & 783 \\
\hline & & 274393 & 100.000 & 26356
\end{tabular}


4-(tert-Butyl)- $N$-(2,6-difluoro-4-(methylsulfonamido)benzyl)benzamide (9)<smiles>CC(C)(C)c1ccc(C(=O)NCc2c(F)cc(NS(C)(=O)=O)cc2F)cc1</smiles>

\section{$254 \mathrm{~nm}$}

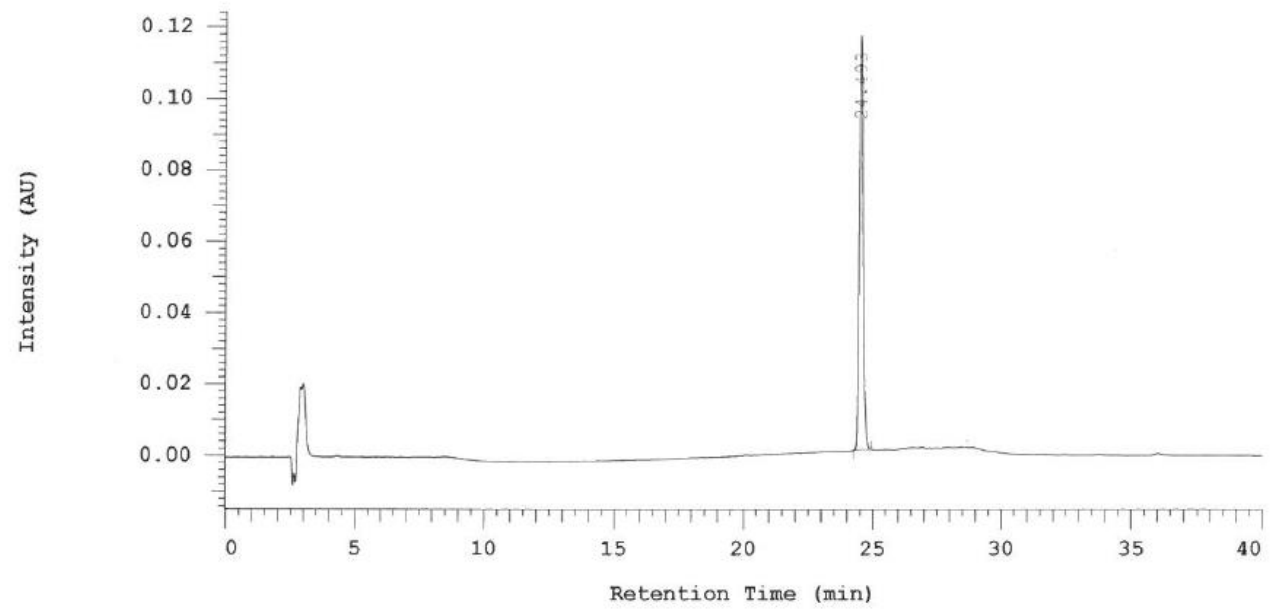

Pump 1: 5160

Pump 1 Solvent $\mathrm{A}$ : $\mathrm{MeOH}$

Pump 1 Solvent $\mathrm{C}$ : $\mathrm{MeOH}$

Pump 1 Solvent B: Wasser

Pump 1 Solvent D: $\mathrm{MeOH}$

\begin{tabular}{ccccr} 
No. & RT & Area & Area \& & Height \\
\hline 1 & 24.493 & 597017 & 100.000 & 58072 \\
\hline & 597017 & 100.000 & 58072 \\
\hline
\end{tabular}

\section{$280 \mathrm{~nm}$}

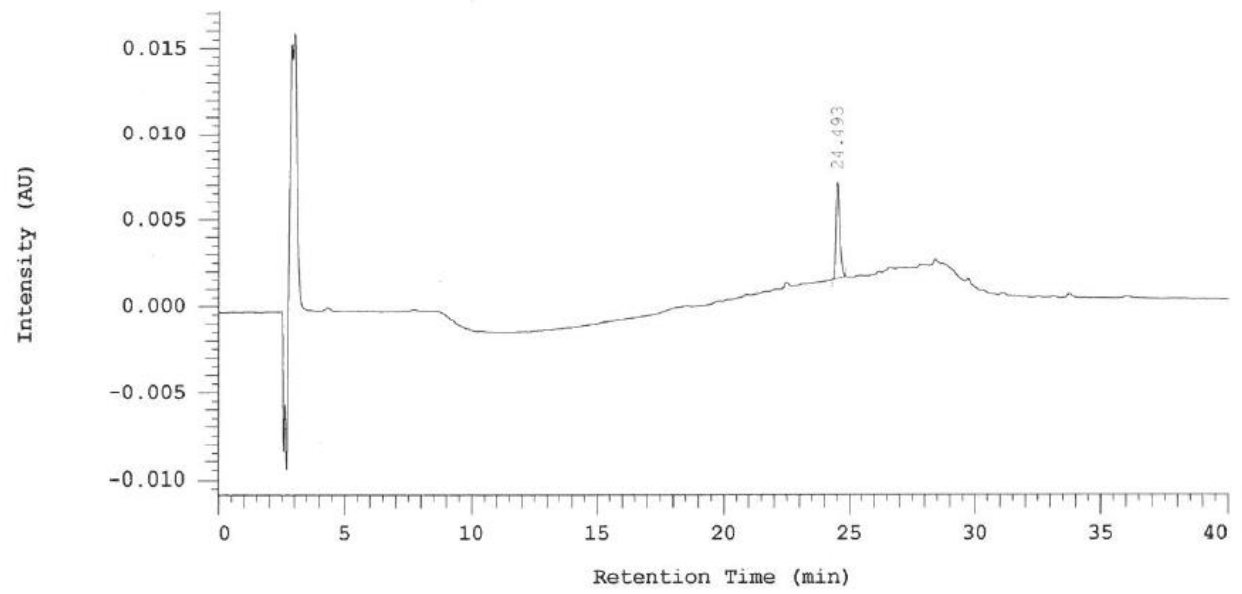

Pump 1: 5160

Pump 1 Solvent A: $\mathrm{MeOH}$

Pump 1 Solvent $\mathrm{C}$ : $\mathrm{MeOH}$

Pump 1 Solvent B: Wasser

Pump 1 Solvent D: $\mathrm{MeOH}$

\begin{tabular}{rrrrr} 
No. & RT & Area & Area : & Height \\
\hline 1 & 24.493 & 28866 & 100.000 & 2767 \\
\hline & 28866 & 100.000 & 2767 \\
\hline
\end{tabular}


<smiles>CC(C)(C)c1ccc(C(=O)NCc2c(Cl)cc(NS(C)(=O)=O)cc2Cl)cc1</smiles>

$254 \mathrm{~nm}$

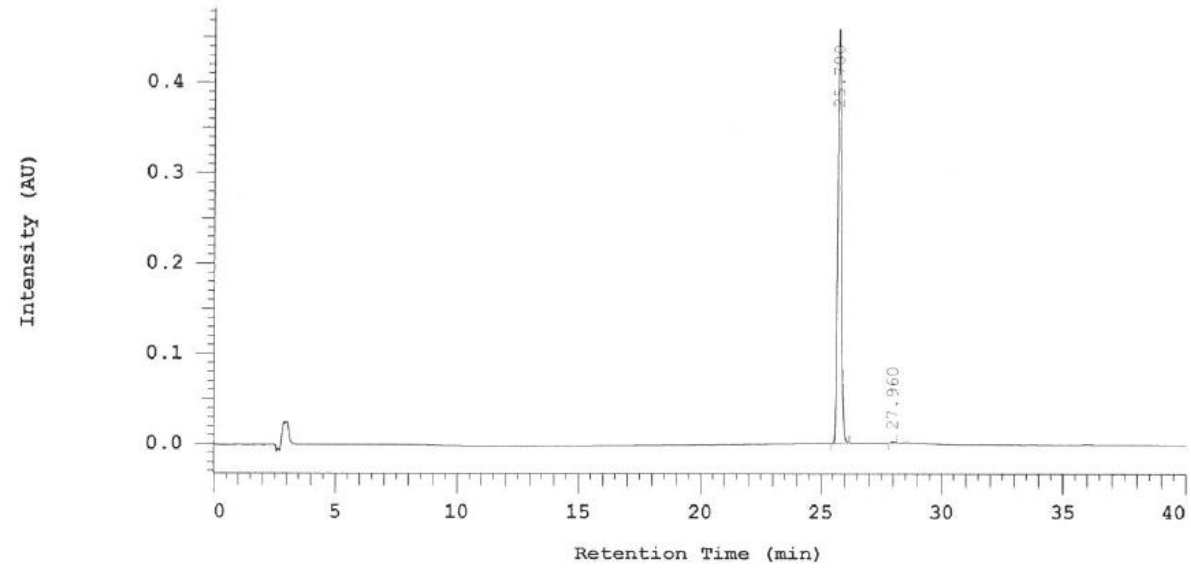

Pump 1: 5160

Pump 1 Solvent A: MeOH Pump 1 Solvent B: Wasser

Pump 1 Solvent $\mathrm{C}: \mathrm{MeOH}$

Pump 1 Solvent D: $\mathrm{MeOH}$

\begin{tabular}{rrrrr} 
No. & RT & Area & Area & Height \\
\hline 1 & 25.700 & 2328905 & 99.789 & 228880 \\
2 & 27.960 & 4928 & 0.211 & 600 \\
\hline & 2333833 & 100.000 & 229480
\end{tabular}

\section{$280 \mathrm{~nm}$}

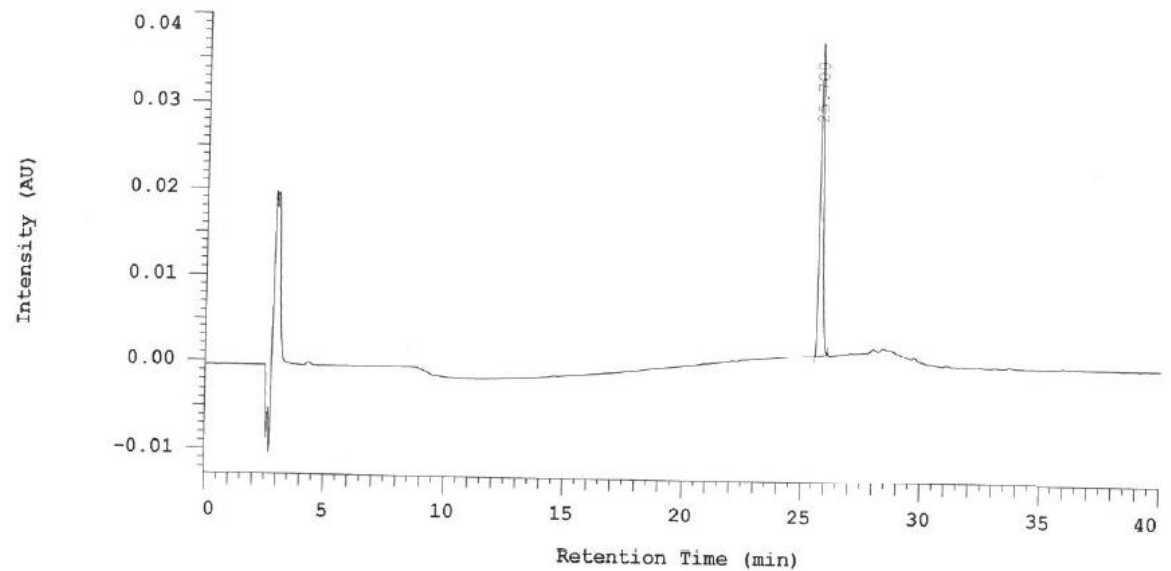

Pump 1: 5160

Pump 1 Solvent A: $\mathrm{MeOH}$

Pump 1 Solvent C: $\mathrm{MeOH}$

Pump 1 Solvent B: Wasser

Pump 1 Solvent D: $\mathrm{MeOH}$

\begin{tabular}{ccccr} 
No. & RT & Area & Area \% & Height \\
\hline 1 & 25.700 & 182548 & 100.000 & 17910 \\
\hline & 182548 & 100.000 & 17910
\end{tabular}


<smiles>CS(=O)(=O)Nc1ccc(CNC(=O)c2ccc(C3(C(F)(F)F)CC3)cc2)c(Cl)c1</smiles>

$254 \mathrm{~nm}$

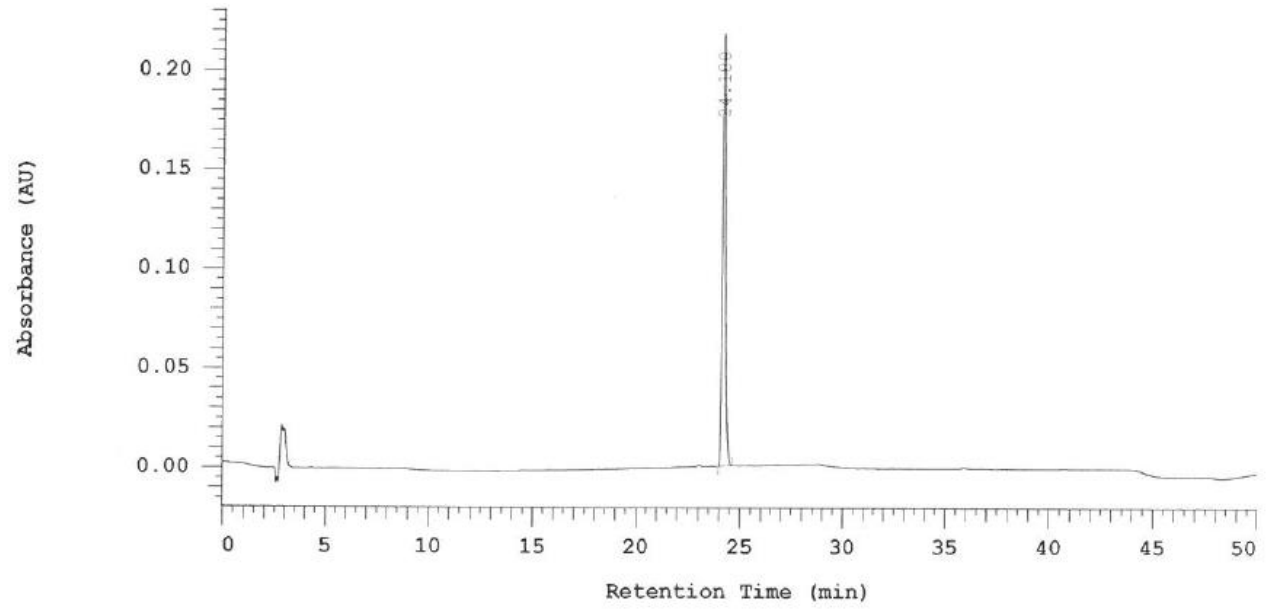

Pump 1: 5160

Pump 1 Solvent $\mathrm{A}$ : $\mathrm{MeOH} \quad$ Pump 1 Solvent B: Wasser

Pump 1 Solvent C: MeOH Pump 1 Solvent D: MeOH

\begin{tabular}{ccccc} 
No. & RT & Area & Area \& & Height \\
\hline 1 & 24.180 & 1090551 & 100.000 & 108721 \\
\hline & & 1090551 & 100.000 & 108721
\end{tabular}

\section{$280 \mathrm{~nm}$}

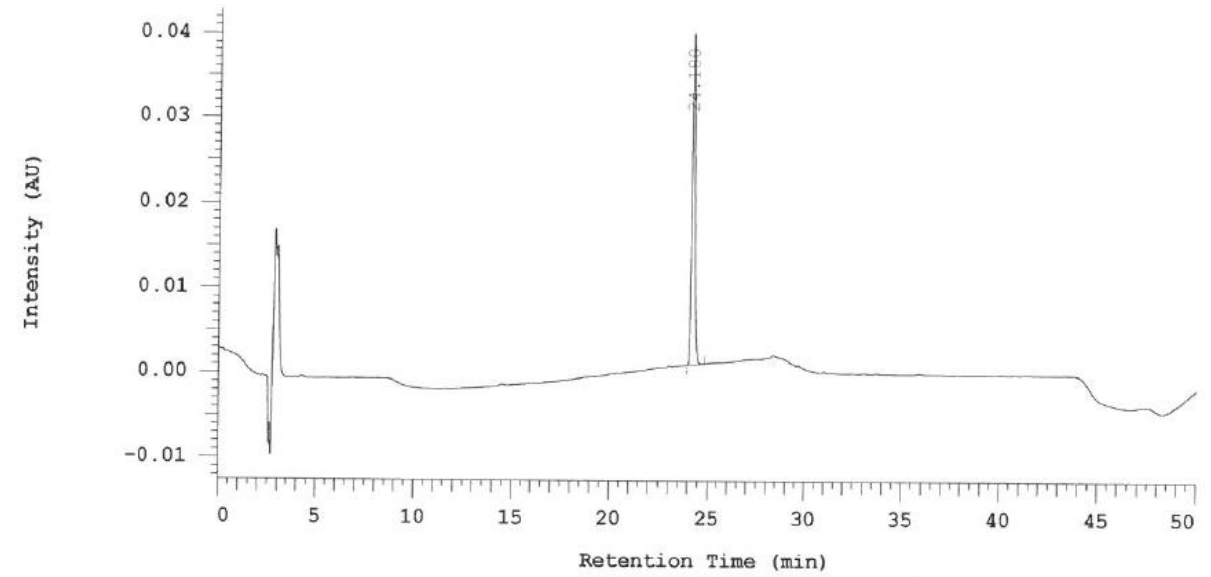

Pump 1: 5160

Pump 1 Solvent A: $\mathrm{MeOH}$

Pump 1 Solvent C: $\mathrm{MeOH}$

Pump 1 Solvent B: Wasser

Pump 1 Solvent D: $\mathrm{MeOH}$

\begin{tabular}{ccccr} 
No. & RT & Area & Area \% & Height \\
\hline 1 & 24.180 & 197432 & 100.000 & 19553 \\
\hline & & 197432 & 100.000 & 19553 \\
\hline
\end{tabular}


<smiles>CS(=O)(=O)Nc1ccc(CNC(=O)c2ccc(C34CCC3C4)cc2)c(Cl)c1</smiles>

\section{$254 \mathrm{~nm}$}

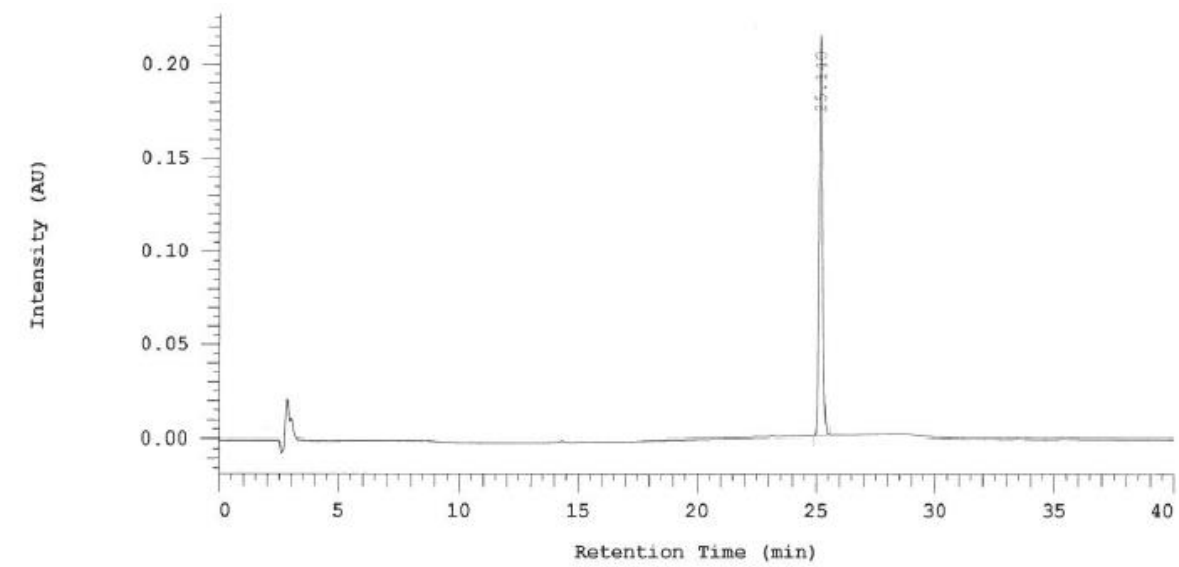

Pump 1: 5160

Pump 1 Solvent A: $\mathrm{MeOH} \quad$ Pump 1 solvent B: Wasser

Pump 1 Solvent $\mathrm{C}: \mathrm{MeOH} \quad$ Pump 1 Solvent D: MeOH

\begin{tabular}{ccccc} 
No. & RT & Area & Area & Height \\
\hline 1 & 25.140 & 1064664 & 100.000 & 107030 \\
\hline & & 1064664 & 100.000 & 107030
\end{tabular}

\section{$280 \mathrm{~nm}$}

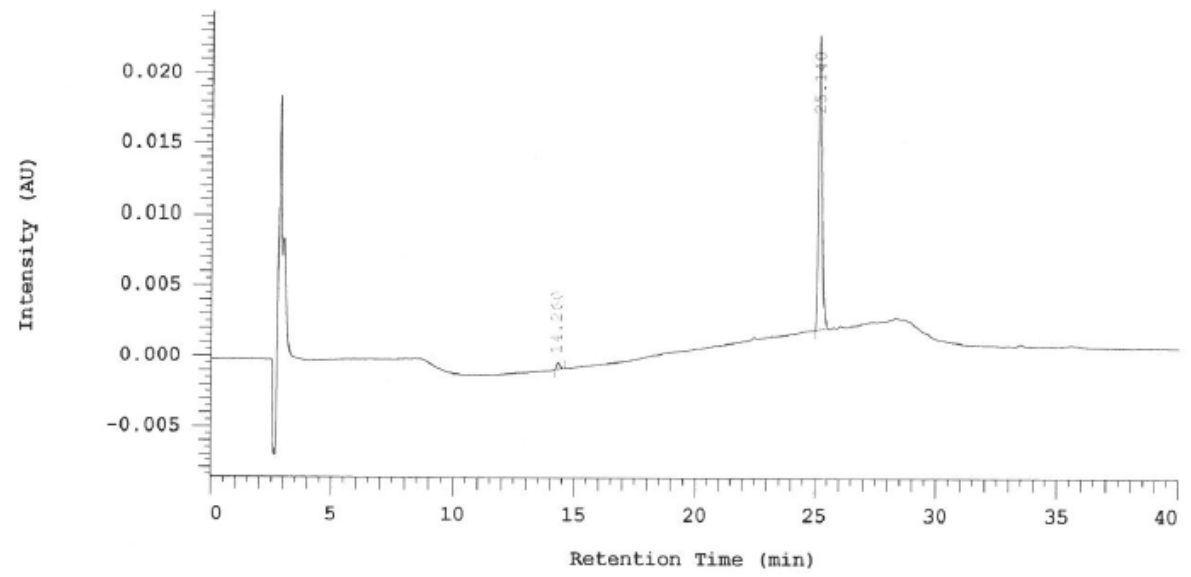

Pump 1: 5160

Pump 1 Solvent A: $\mathrm{MeOH}$

Pump 1 Solvent $\mathrm{C}$ : $\mathrm{MeOH}$

Pump 1 Solvent B: Wasser

Pump 1 Solvent D: $\mathrm{MeOH}$

\begin{tabular}{rrrrr} 
No. & RT & Area & Area 8 & Height \\
\hline 1 & 14.260 & 2521 & 2.382 & 141 \\
2 & 25.140 & 103332 & 97.618 & 10401 \\
\hline & & 105853 & 100.000 & 10542
\end{tabular}


<smiles>CS(=O)(=O)Nc1ccc(CNC(=O)c2ccc(C(F)(F)F)cc2)c(Cl)c1</smiles>

\section{$254 \mathrm{~nm}$}

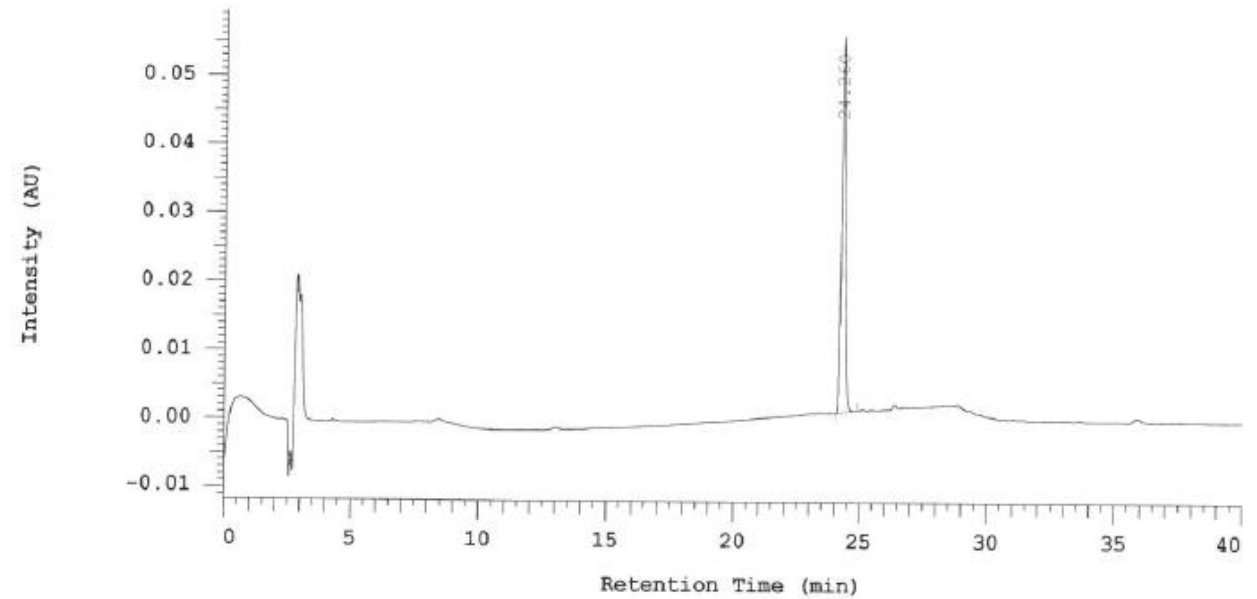

Pump 1: 5160

Pump 1 Solvent A: $\mathrm{MeOH}$

Pump 1 Solvent C: MeOH

Pump 1 Solvent B: Wasser

Pump 1 Solvent D: $\mathrm{MeOH}$

\begin{tabular}{ccccc} 
No. & RT & Area & Area \& & Height \\
\hline 1 & 24.260 & 276269 & 100.000 & 27400 \\
\hline & & 276269 & 100.000 & 27400
\end{tabular}

\section{$280 \mathrm{~nm}$}

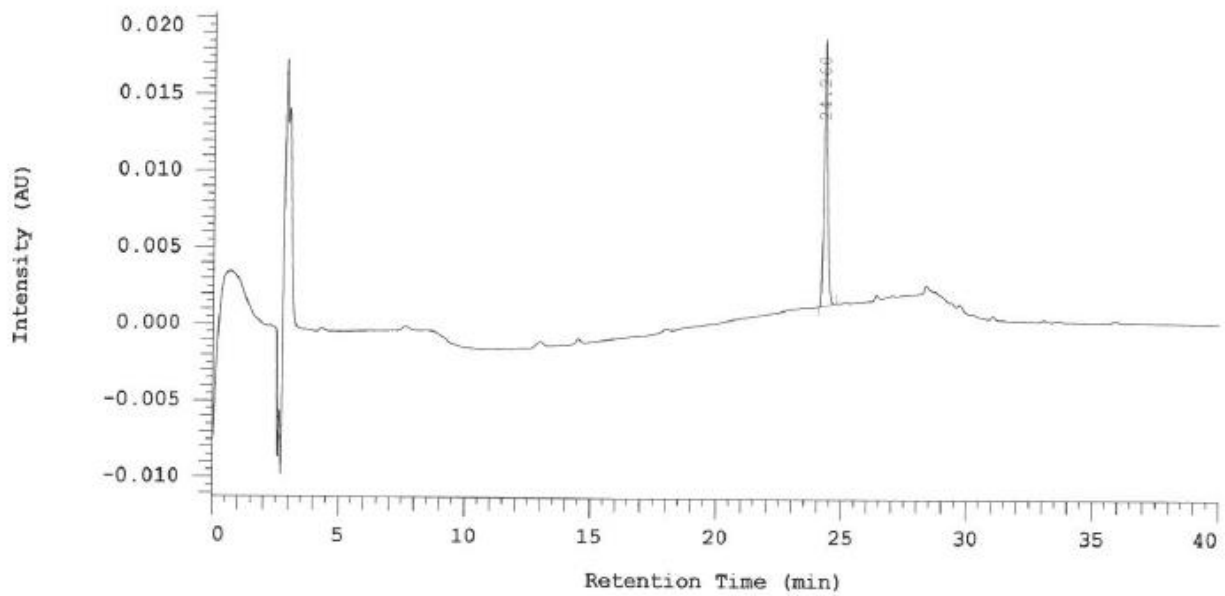

Pump 1: 5160

Pump 1 Solvent A: $\mathrm{MeOH}$

Pump 1 Solvent C: $\mathrm{MeOH}$

Pump 1 Solvent B: Wasser

Pump 1 Solvent D: $\mathrm{MeOH}$

\begin{tabular}{ccccr} 
No. & RT & Area & Area 응 & Height \\
\hline 1 & 24.260 & 87702 & 100.000 & 8702 \\
\hline & & 87702 & 100.000 & 8702
\end{tabular}


<smiles>CN(C)c1ccc(C(=O)NCc2ccc(NS(C)(=O)=O)cc2Cl)cc1</smiles>

\section{$254 \mathrm{~nm}$}

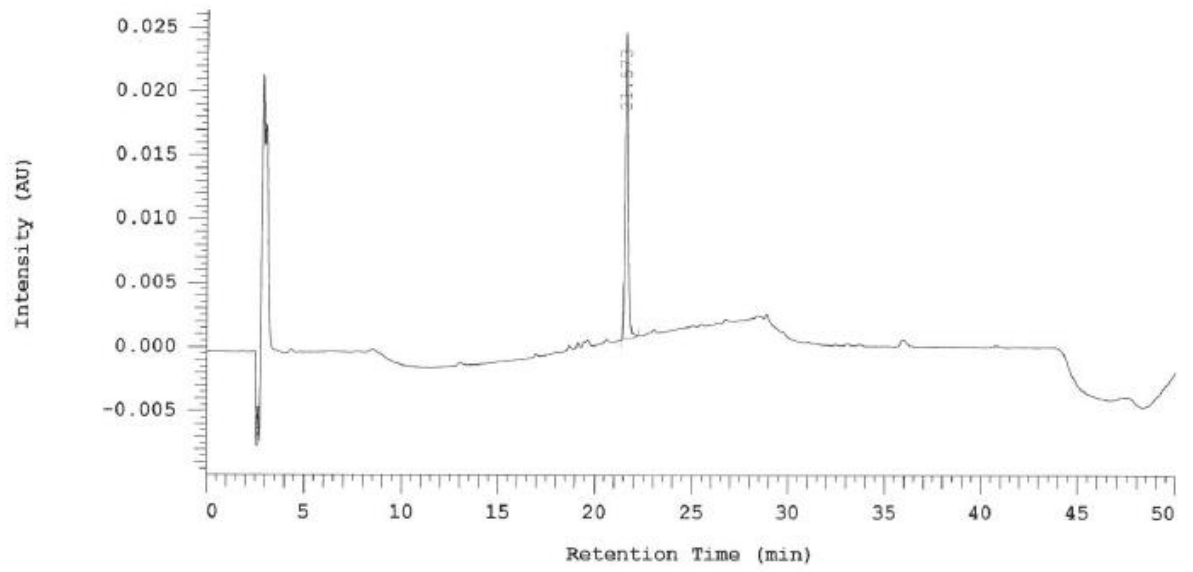

Pump 1: 5160

Pump 1 Solvent A: MeOH Pump 1 Solvent B: Wasser

Pump 1 Solvent $\mathrm{C}$ : $\mathrm{MeOH}$

Pump 1 Solvent B: Wasser
Pump 1 Solvent D: MeOH

\begin{tabular}{rrrrr} 
No. & RT & Area & Area & Height \\
\hline 1 & 21.573 & 129045 & 100.000 & 11986 \\
\hline & 129045 & 100.000 & 11986
\end{tabular}

\section{$280 \mathrm{~nm}$}

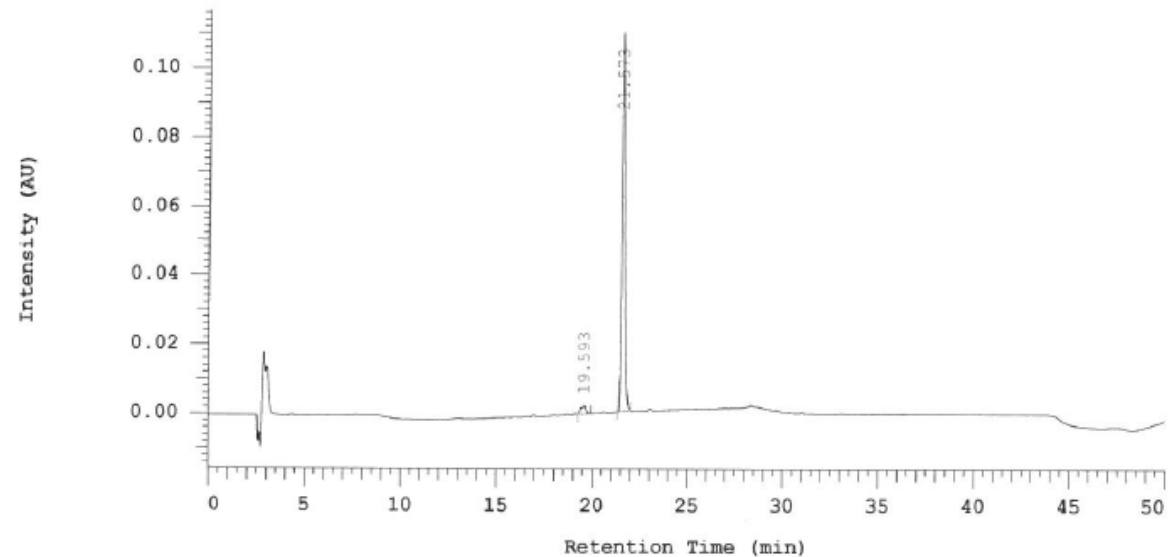

Pump 1: 5160

Pump 1 Solvent A: $\mathrm{MeOH}$

Pump 1 Solvent $\mathrm{C}$ : $\mathrm{MeOH}$

Pump 1 Solvent B: Wasser

Pump 1 Solvent D: $\mathrm{MeOH}$

\begin{tabular}{rrrrr} 
No. & RT & Area & Area & Height \\
\hline 1 & 19.593 & 20857 & 3.461 & 1222 \\
2 & 21.573 & 581737 & 96.539 & 54800 \\
\hline & & 602594 & 100.000 & 56022
\end{tabular}


<smiles>COc1ccc(C(=O)NCc2ccc(NS(C)(=O)=O)cc2Cl)cc1</smiles>

\section{$254 \mathrm{~nm}$}

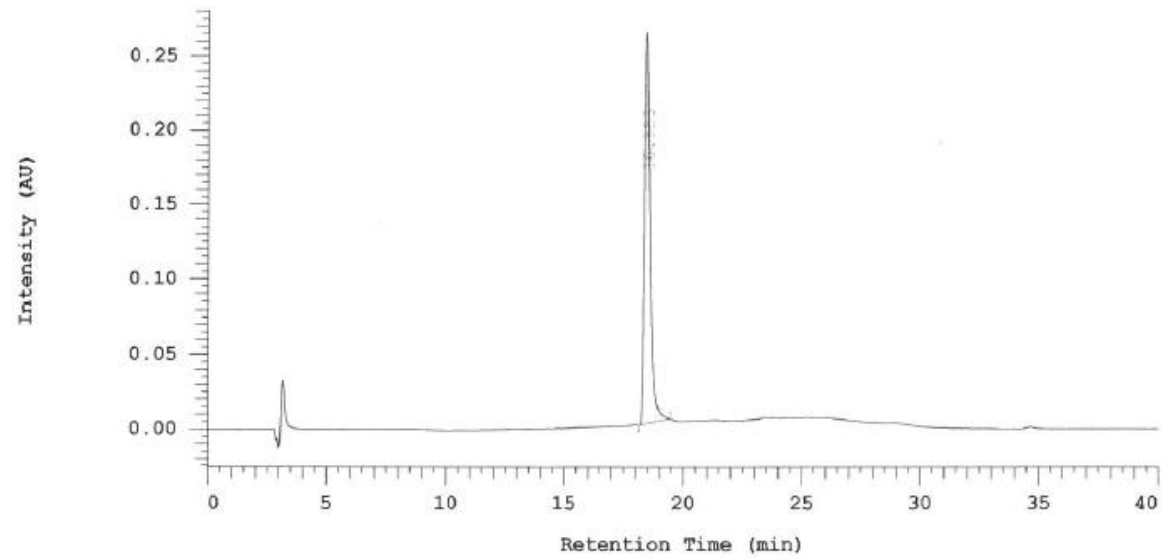

Pump 1: 5160

Pump 1 Solvent A: MeOH Pump 1 Solvent B: Wasser

Pump 1 Solvent C: $\mathrm{MeOH}$

Pump 1 Solvent D: $\mathrm{MeOH}$

\begin{tabular}{ccccr} 
No. & RT & Area & Area : & Height \\
\hline 1 & 18.553 & 2121768 & 100.000 & 80460 \\
\hline & 2121768 & 100.000 & 80460
\end{tabular}

\section{$280 \mathrm{~nm}$}

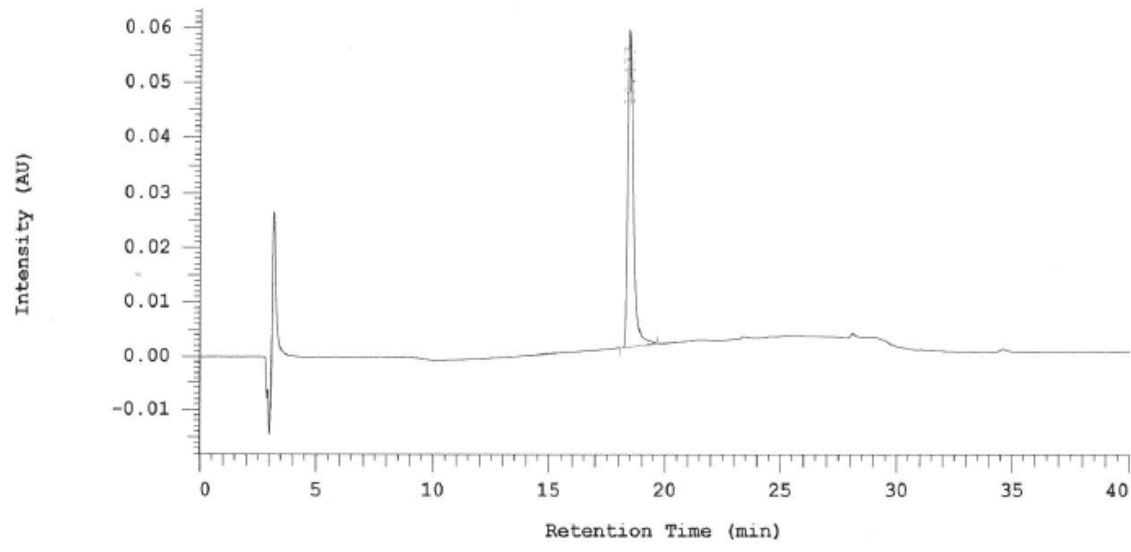

Pump 1: 5160

Pump 1 Solvent A: MeO

Pump 1 Solvent C: $\mathrm{MeOH}$

Pump 1 Solvent B: Wasser

Pump 1 Solvent D: $\mathrm{MeOH}$

\begin{tabular}{ccccc} 
No. & RT & Area & Area & Height \\
\hline 1 & 18.433 & 476938 & 100.000 & 28860 \\
\hline & & 476938 & 100.000 & 28860
\end{tabular}


4-(Azetidin-1-yl)-N-(2-chloro-4-(methylsulfonamido)benzyl)benzamide (16)<smiles>CS(=O)(=O)Nc1ccc(CNC(=O)c2ccc(N3CCC3)cc2)c(Cl)c1</smiles>

\section{$254 \mathrm{~nm}$}

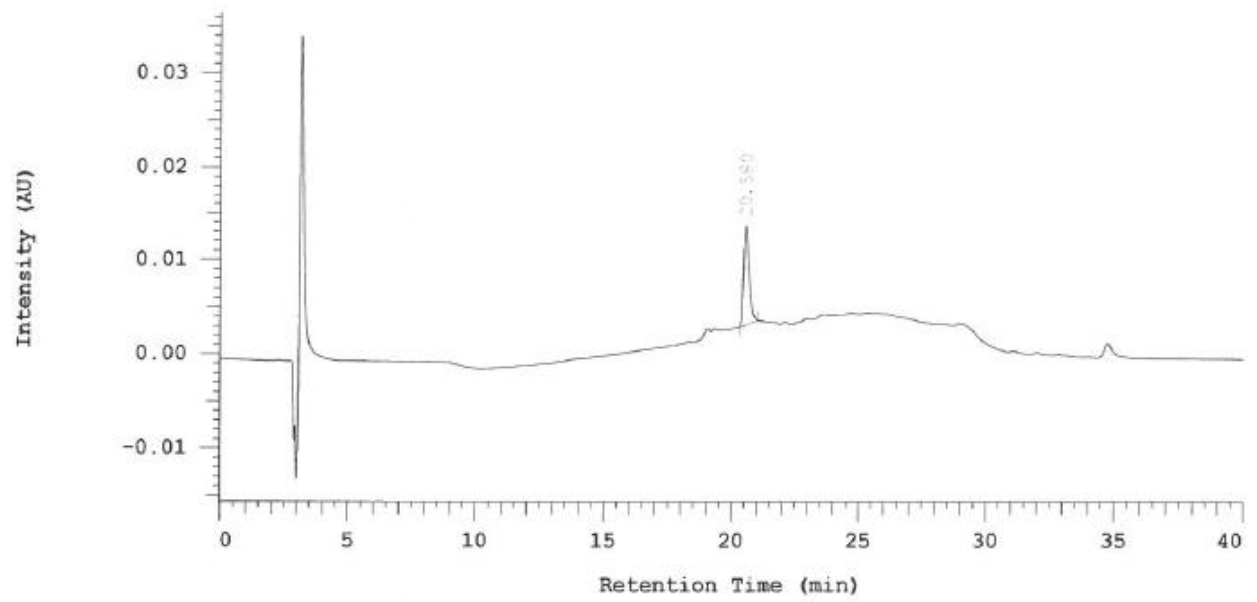

Pump 1: 5160

Pump 1 Solvent A: $\mathrm{MeOH}$

Pump 1 Solvent C: $\mathrm{MeOH}$

Pump 1 Solvent B: Wasser

Pump 1 Solvent D: MeOH

\begin{tabular}{ccccr} 
No. & RT & Area & Area \& & Height \\
\hline 1 & 20.580 & 77740 & 100.000 & 5039 \\
\hline & & 77740 & 100.000 & 5039
\end{tabular}

\section{$280 \mathrm{~nm}$}

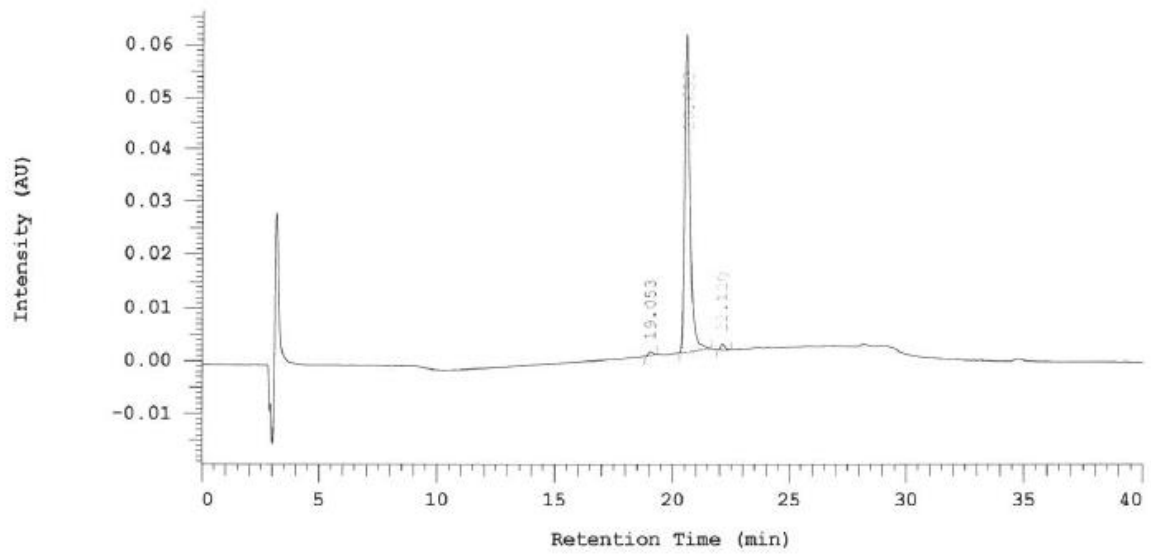

Pump 1: 5160

Pump 1 Solvent A: MeOH

Pump 1 Solvent $\mathrm{C}$ : $\mathrm{MeOH}$

Pump 1 Solvent B: Wasser

Pump 1 Solvent D: $\mathrm{MeOH}$

\begin{tabular}{rrrrr} 
No. & RT & Area & Area \& & Height \\
\hline 1 & 19.053 & 4973 & 1.005 & 365 \\
2 & 20.653 & 482274 & 97.475 & 19792 \\
3 & 22.120 & 7519 & 1.520 & 565 \\
\hline & & 494766 & 100.000 & 20722
\end{tabular}


4-(Pyrrolidine)-N-(2-chloro-4-(methylsulfonamido)benzyl)benzamide (17)<smiles>CS(=O)(=O)Nc1ccc(CNC(=O)c2ccc(N3CCCC3)cc2)c(Cl)c1</smiles>

$254 \mathrm{~nm}$

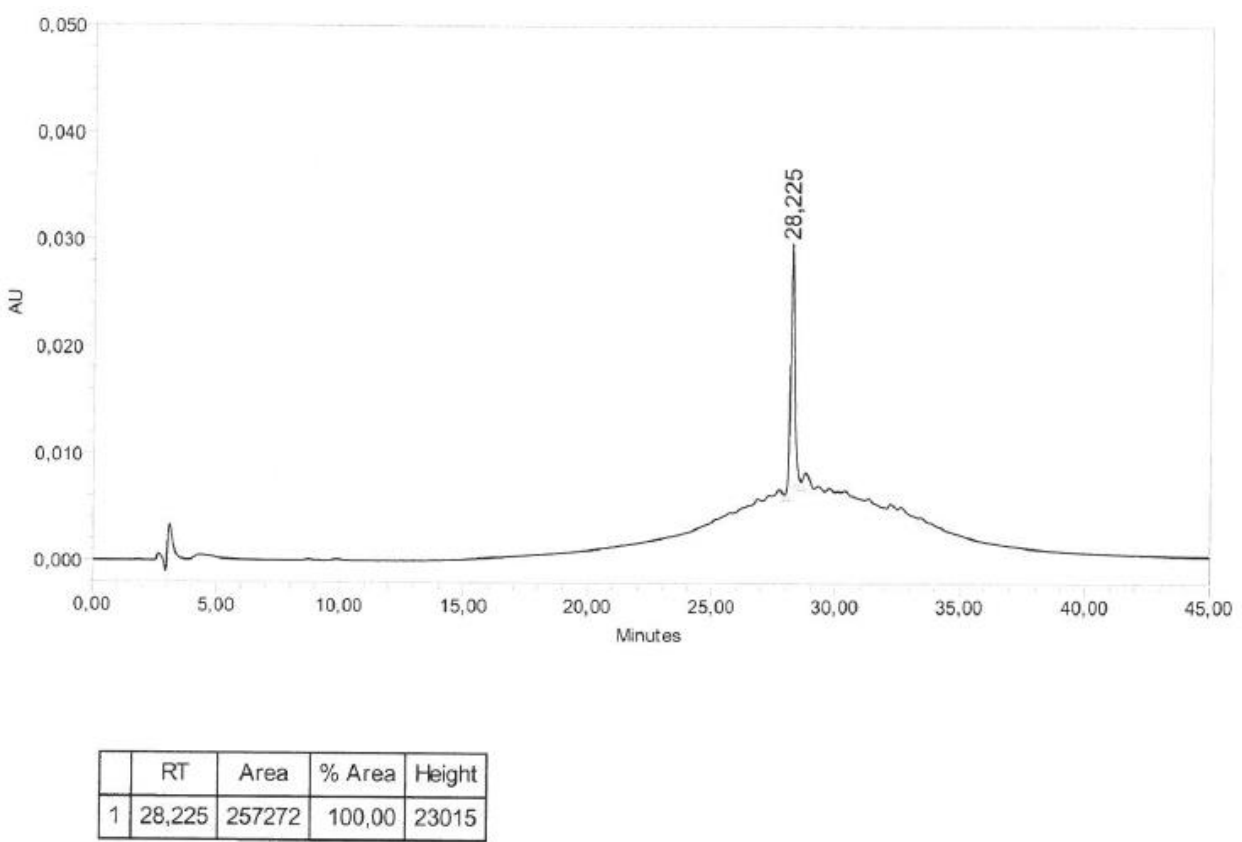

$280 \mathrm{~nm}$

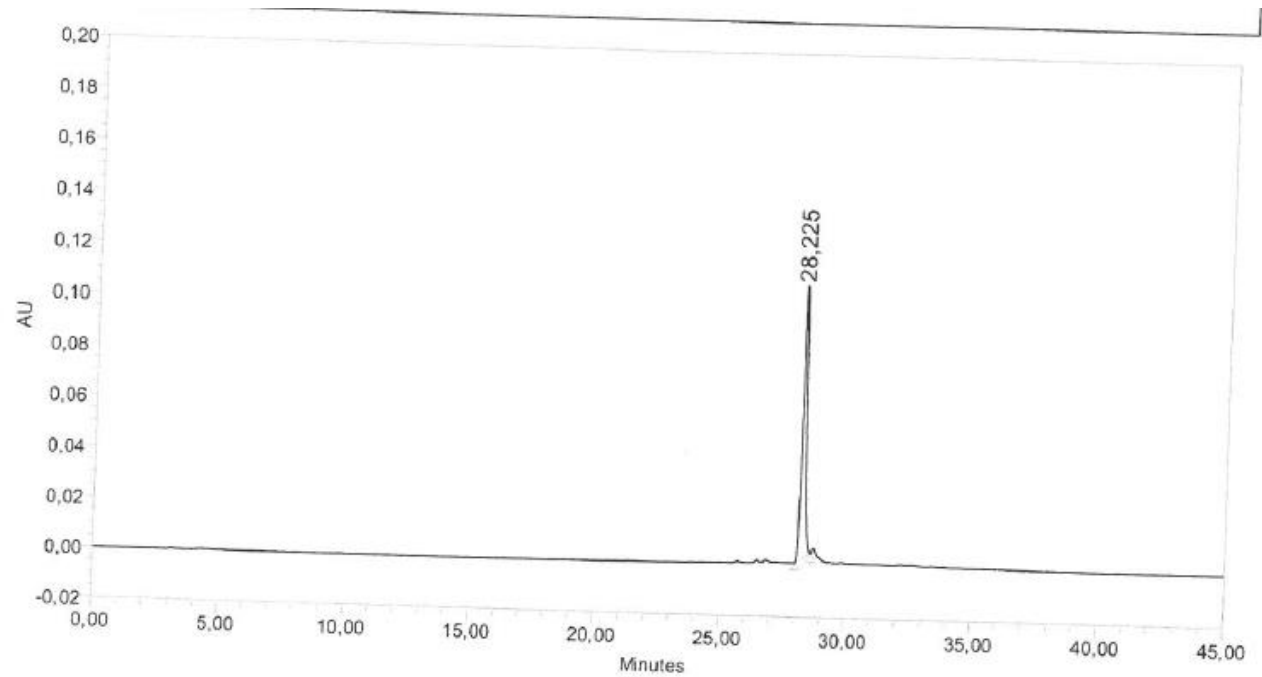

\begin{tabular}{|c|c|c|c|c|}
\hline & RT & Area & $\%$ Area & Height \\
\hline 1 & 28,225 & 1179324 & 100,00 & 107452 \\
\hline
\end{tabular}


4-(Piperidin-1-yl)-N-(2-chloro-4-(methylsulfonamido)benzyl)benzamide (18)<smiles>CS(=O)(=O)Nc1ccc(CNC(=O)c2ccc(N3CCCCC3)cc2)c(Cl)c1</smiles>

$254 \mathrm{~nm}$

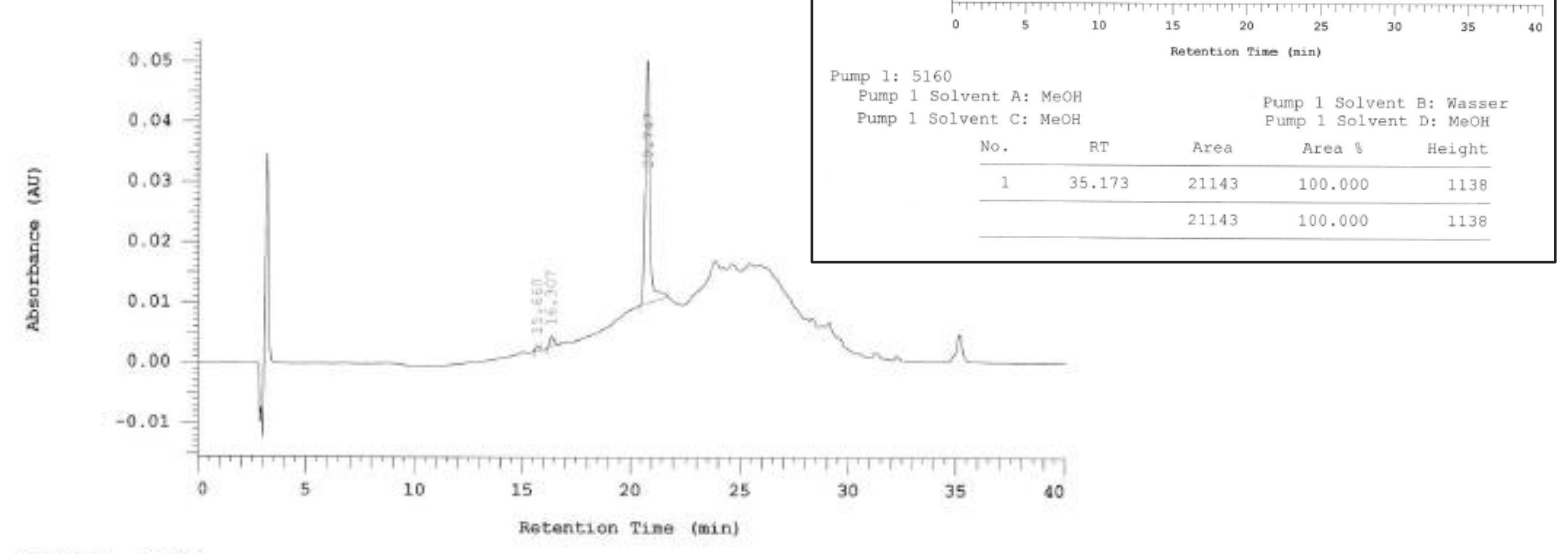

Pump 1: 5160

Bump 1 Solvent A: MeOH Pump 1 Solvent. B: Wasser

Pump 1 Solvent C: $\mathrm{MeOH}$

Pump I Solvent D: MeOH

\begin{tabular}{rrrrr} 
No. & \multicolumn{1}{c}{ RT } & \multicolumn{1}{c}{ Area } & Area \& & Height \\
\hline 1 & 15.660 & 4783 & 1.614 & 434 \\
2 & 16.307 & 9955 & 3.359 & 829 \\
3 & 20.747 & 281674 & 95.028 & 17056 \\
\hline & & 296412 & 100.000 & 18319 \\
\hline
\end{tabular}

$280 \mathrm{~nm}$

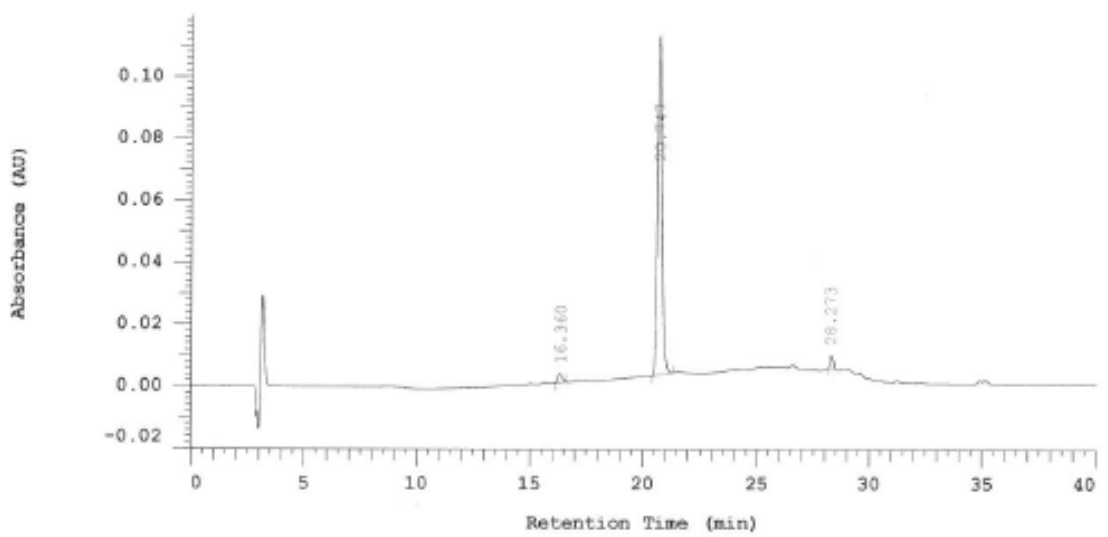

Pump 1: 5160

Pump 1 Solvent A: $\mathrm{MeOH}$

Pump 1 Solvent C: $\mathrm{MeOH}$

Pump 1 Solvent B: Wasser

Pump 1 Solvent D: $\mathrm{MeOH}$

\begin{tabular}{rrrrr} 
No. & RT & \multicolumn{1}{c}{ Area } & Area \& & Height \\
\hline 1 & 16.360 & 18417 & 2.509 & 1257 \\
2 & 20.747 & 697472 & 95.005 & 46242 \\
3 & 28.273 & 18253 & 2.486 & 2016 \\
\hline & & 734142 & 100.000 & 49515
\end{tabular}


<smiles>CS(=O)(=O)Nc1ccc(CNC(=O)c2ccc(N3CCOCC3)cc2)c(Cl)c1</smiles>

\section{$254 \mathrm{~nm}$}

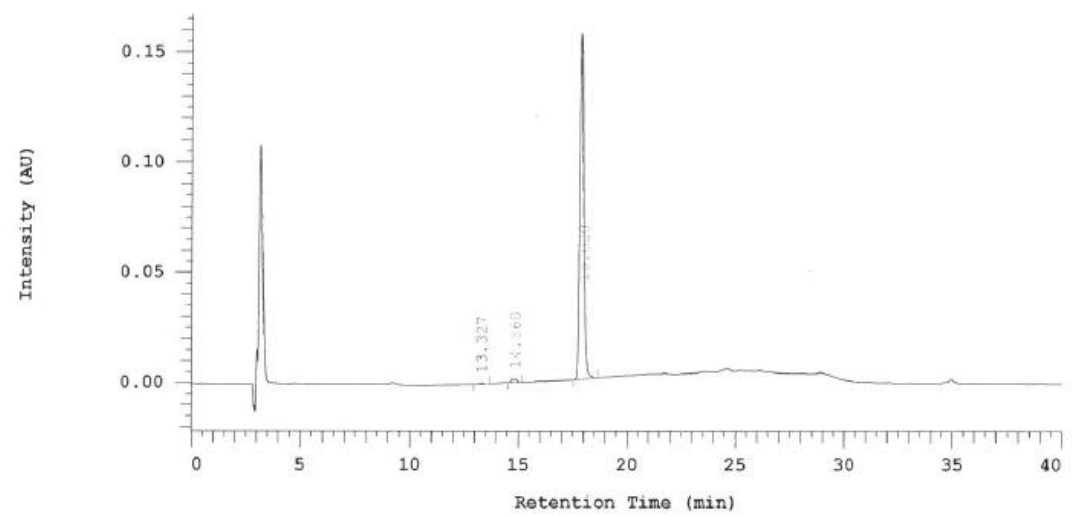

Pump 1: 5160

Pump 1 Solvent A: MeOH Pump 1 Solvent B: Wasser

Pump 1 Solvent C: $\mathrm{MeOH}$

Pump 1 Solvent D: $\mathrm{MeOH}$

\begin{tabular}{rrrrr} 
No. & RT & \multicolumn{1}{c}{ Area } & Area 8 & Height \\
\hline 1 & 13.327 & 3118 & 0.338 & 187 \\
2 & 14.860 & 13215 & 1.435 & 741 \\
3 & 18.020 & 904889 & 98.227 & 19752 \\
\hline & & 921222 & 100.000 & 20680
\end{tabular}

$280 \mathrm{~nm}$

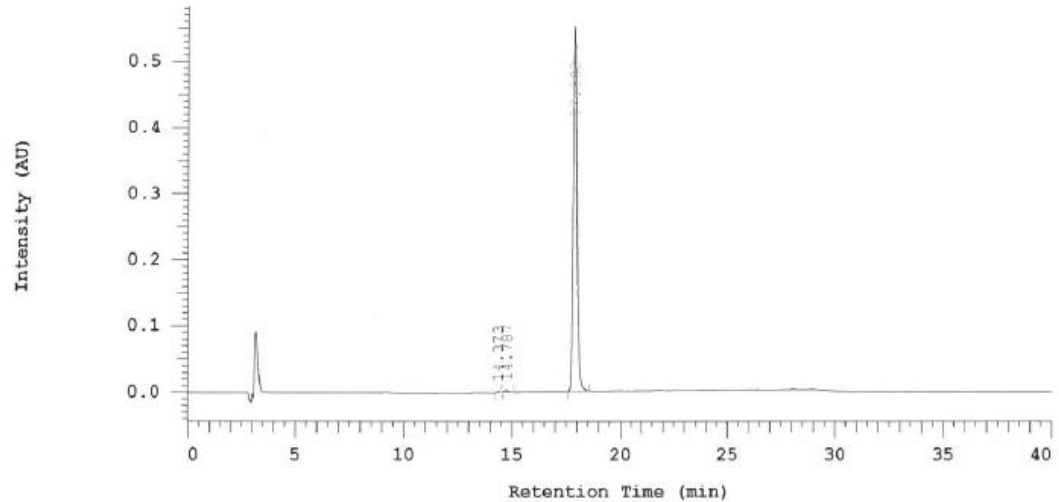

Pump 1: 5160

Pump 1 Solvent A: $\mathrm{MeO}$

Pump 1 Solvent C: $\mathrm{MeOH}$

Pump 1 Solvent $B$ : Wasser

\begin{tabular}{rrrrr} 
No. & RT & \multicolumn{1}{c}{ Area } & Area 8 & Height \\
\hline 1 & 14.373 & 3324 & 0.104 & 357 \\
2 & 14.787 & 18615 & 0.580 & 1182 \\
3 & 17.893 & 3190089 & 99.317 & 260537 \\
\hline & & 3212028 & 100.000 & 262076
\end{tabular}


<smiles>CS(=O)(=O)Nc1ccc(CNC(=O)c2ccc(OC(F)(F)F)cc2)c(Cl)c1</smiles>

\section{$254 \mathrm{~nm}$}

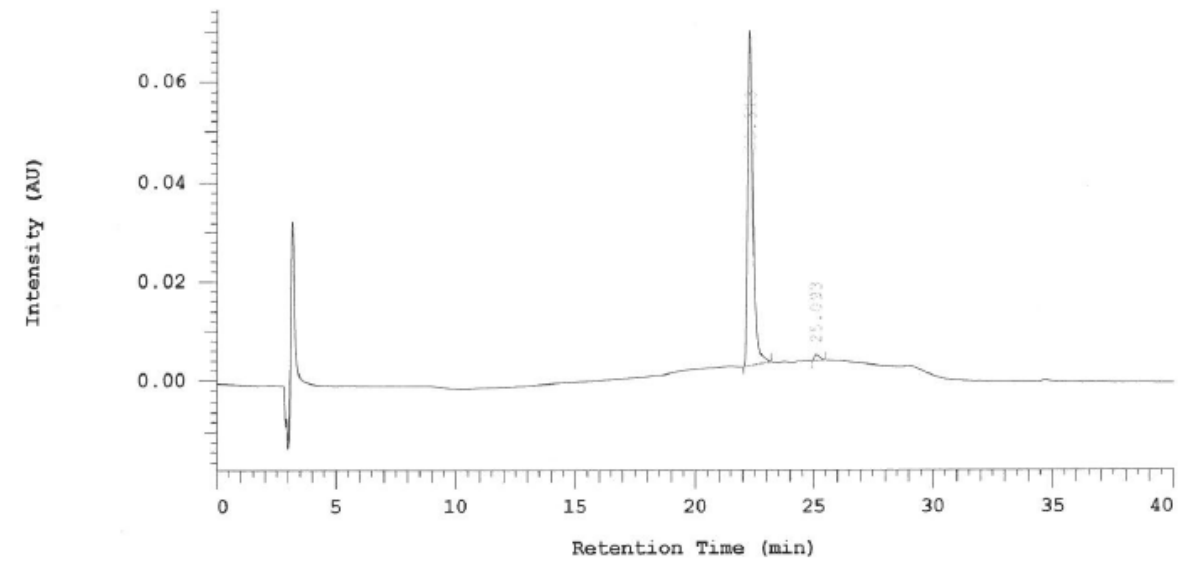

Pump 1: 5160

Pump 1 Solvent A: MeOH Pump 1 Solvent B: Wasser

Pump 1 Solvent C: $\mathrm{MeOH}$

Pump 1 Solvent $\mathrm{D}$ : $\mathrm{MeOH}$

\begin{tabular}{rrrrr} 
No. & RT & Area & Area : & Height \\
\hline 1 & 22.313 & 520848 & 98.336 & 29160 \\
2 & 25.093 & 8813 & 1.664 & 589 \\
\hline & & 529661 & 100.000 & 29749 \\
\hline
\end{tabular}

$280 \mathrm{~nm}$

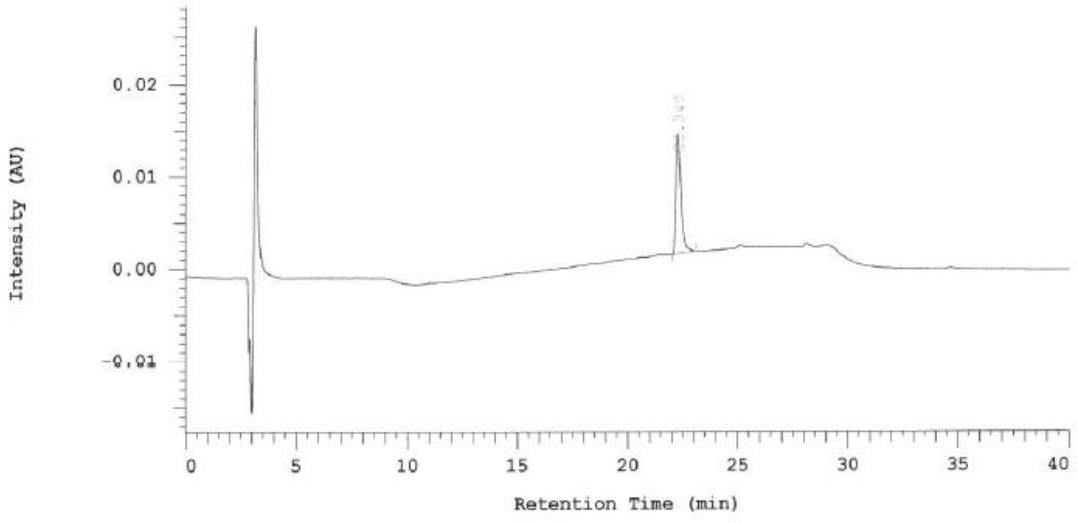

Pump 1: 5160

Pump 1 Solvent A: $\mathrm{MeOH}$

Pump 1 Solvent $\mathrm{C}$ : $\mathrm{MeOH}$

Pump 1 Solvent B: Wasser

Pump 1 Solvent D: MeOH

\begin{tabular}{rcccr} 
No. & RT & Area & Area \& & Height \\
\hline 1 & 22.340 & 100403 & 100.000 & 4898 \\
\hline & & 100403 & 100.000 & 4898 \\
\hline
\end{tabular}


<smiles>CCOc1ccc(C(=O)NCc2ccc(NS(C)(=O)=O)cc2Cl)cc1</smiles>

\section{$254 \mathrm{~nm}$}

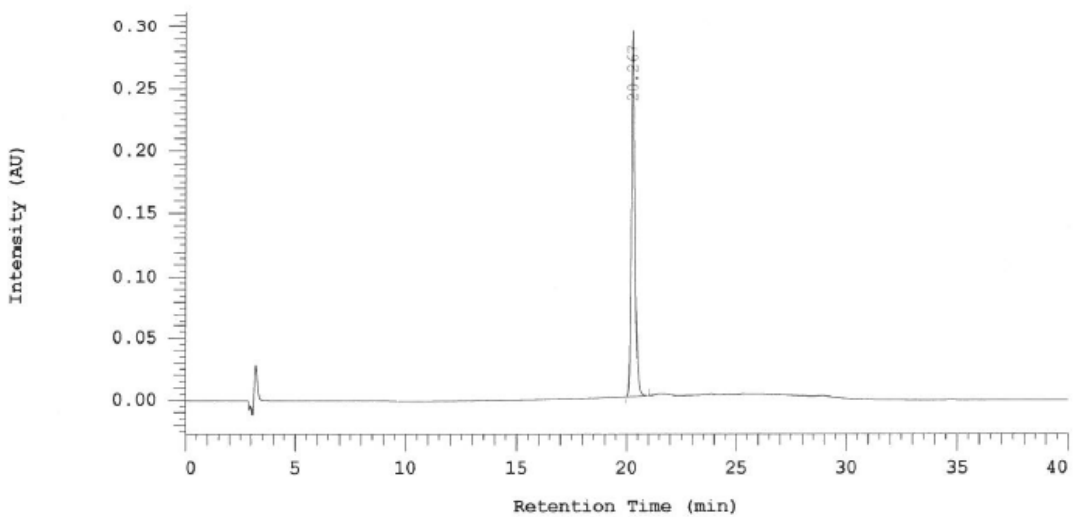

Pump 1: 5160

Pump 1 Solvent A: $\mathrm{MeOH}$

Pump 1 Solvent C: $\mathrm{MeOH}$

Pump 1 Solvent B: Wasser

Pump 1 Solvent D: MeOH

\begin{tabular}{ccccc} 
No. & RT & Area & Area 8 & Height \\
\hline 1 & 20.267 & 1659639 & 100.000 & 146825 \\
\hline & & 1659639 & 100.000 & 146825
\end{tabular}

\section{$280 \mathrm{~nm}$}

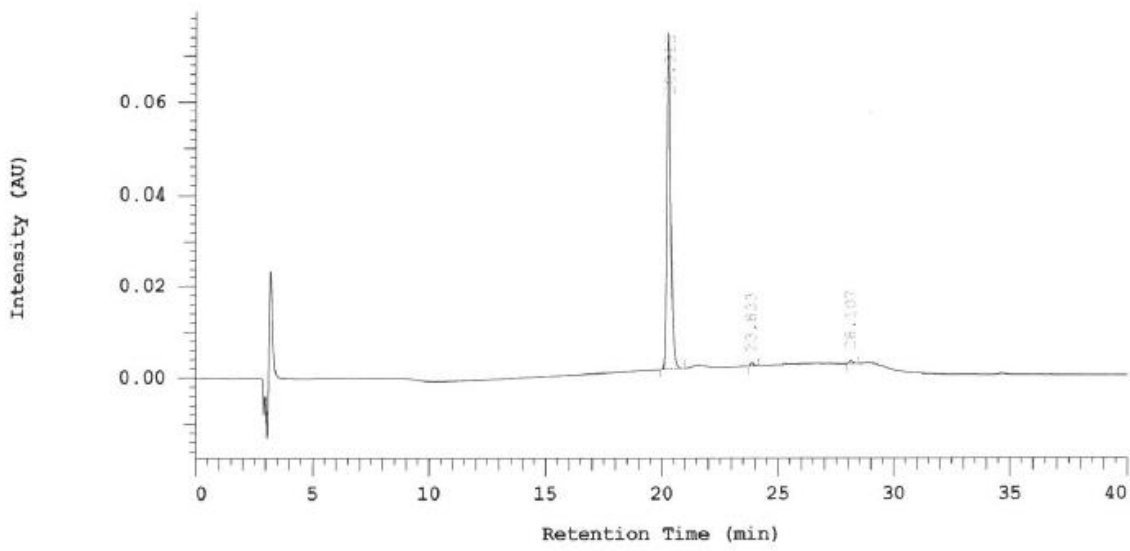

Pump 1: 5160

Pump 1 Solvent $A$ : $\mathrm{MeOH}$

Pump 1 Solvent $\mathrm{C}$ : $\mathrm{MeOH}$

Pump 1 Solvent $B$ : Wasser

\begin{tabular}{rrrrr} 
No. & \multicolumn{1}{c}{ RT } & Area & Area \& & Height \\
\hline 1 & 20.313 & 414625 & 98.545 & 28587 \\
2 & 23.833 & 3049 & 0.725 & 255 \\
3 & 28.107 & 3072 & 0.730 & 297 \\
\hline & & 420746 & 100.000 & 29139
\end{tabular}


<smiles>CC(C)Oc1ccc(C(=O)NCc2ccc(NS(C)(=O)=O)cc2Cl)cc1</smiles>

\section{$254 \mathrm{~nm}$}

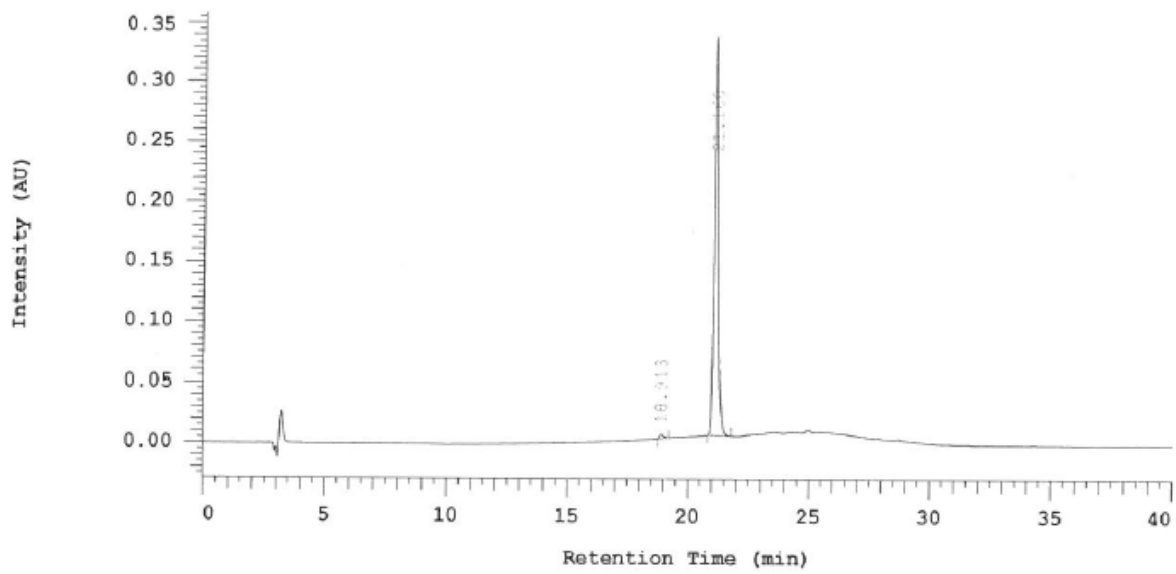

Pump 1: 5160

Pump 1 Solvent A: $\mathrm{MeOH}$

Pump 1 Solvent C: $\mathrm{MeOH}$

Pump 1 Solvent B: Wasser

Pump 1 Solvent D: MeOH

\begin{tabular}{rrrrr} 
No. & R'T & \multicolumn{1}{c}{ Area } & Area \& & Height \\
\hline 1 & 18.913 & 19311 & 1.016 & 1733 \\
2 & 21.160 & 1881171 & 98.984 & 112824 \\
\hline & & 1900482 & 100.000 & 114557 \\
\hline
\end{tabular}

\section{$280 \mathrm{~nm}$}

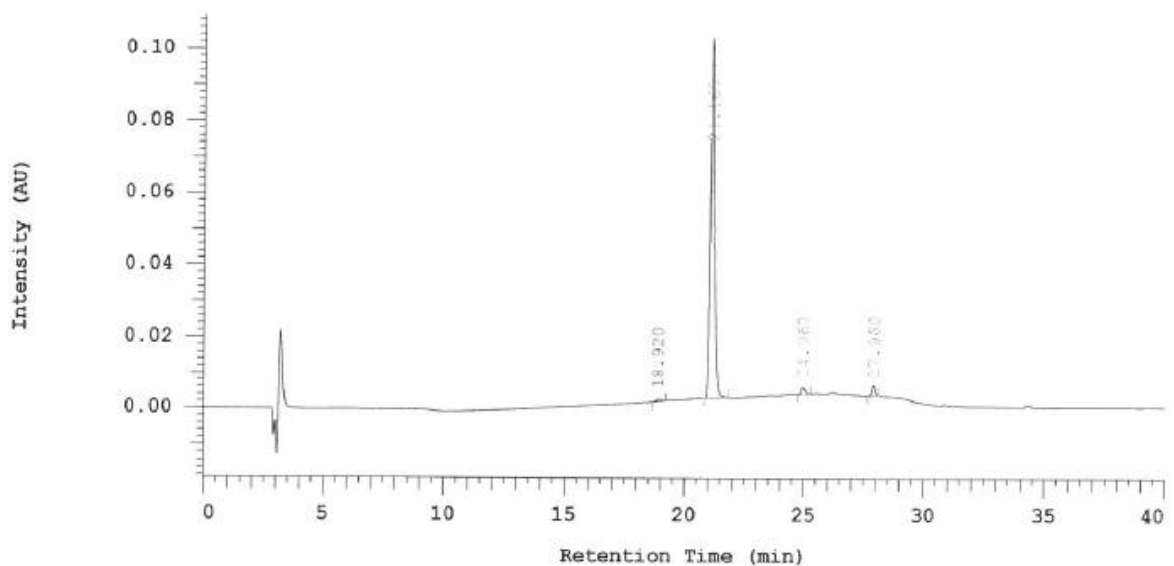

Pump 1: 5160

Pump 1 Solvent A: $\mathrm{MeOH}$

Pump 1 Solvent C: $\mathrm{MeOH}$

Pump 1 Solvent B: Wasser

Pump 1 Solvent D: $\mathrm{MeOH}$

\begin{tabular}{rrrrr} 
No. & RT & \multicolumn{1}{c}{ Area } & Area \& & Height \\
\hline 1 & 18.920 & 3494 & 0.584 & 303 \\
2 & 21.160 & 569873 & 95.308 & 34087 \\
3 & 24.967 & 10562 & 1.766 & 839 \\
4 & 27.960 & 13998 & 2.341 & 1027 \\
\hline & & 597927 & 100.000 & 36256
\end{tabular}


<smiles>CC(C)(O)c1ccc(C(=O)NCc2ccc(NS(C)(=O)=O)cc2Cl)cc1</smiles>

\section{$254 \mathrm{~nm}$}

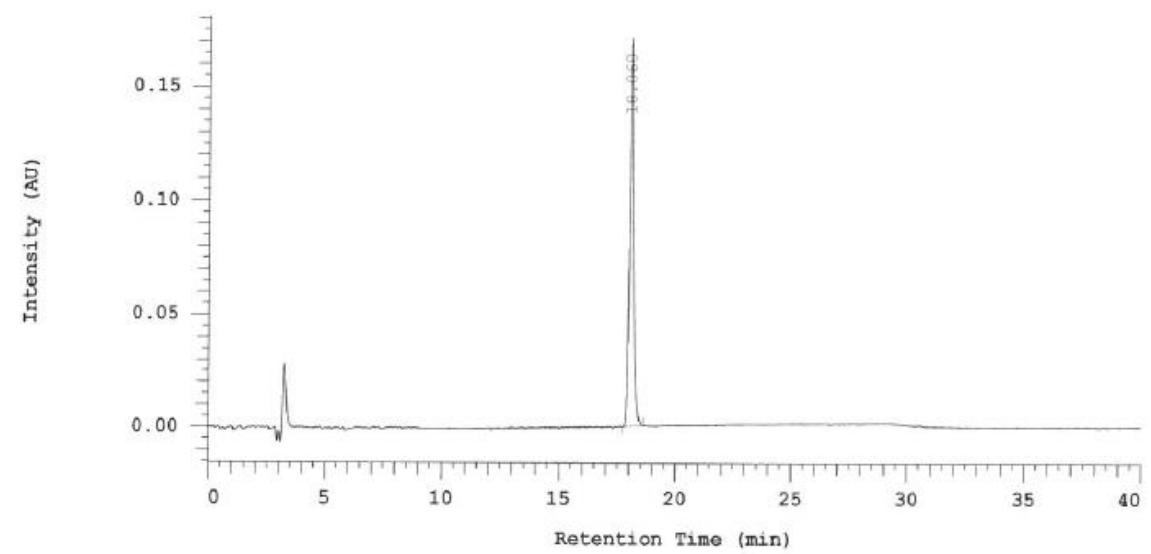

Pump 1: 5160

Pump 1 Solvent A: MeOH

Pump 1 Solvent C: $\mathrm{MeOH}$

Pump 1 Solvent B: Wasser

Pump 1 Solvent D: $\mathrm{MeOH}$

\begin{tabular}{ccccr} 
No. & RT & Area & Area \& & Height \\
\hline 1 & 18.060 & 1016201 & 100.000 & 85415 \\
\hline & & 1016201 & 100.000 & 85415
\end{tabular}

\section{$280 \mathrm{~nm}$}

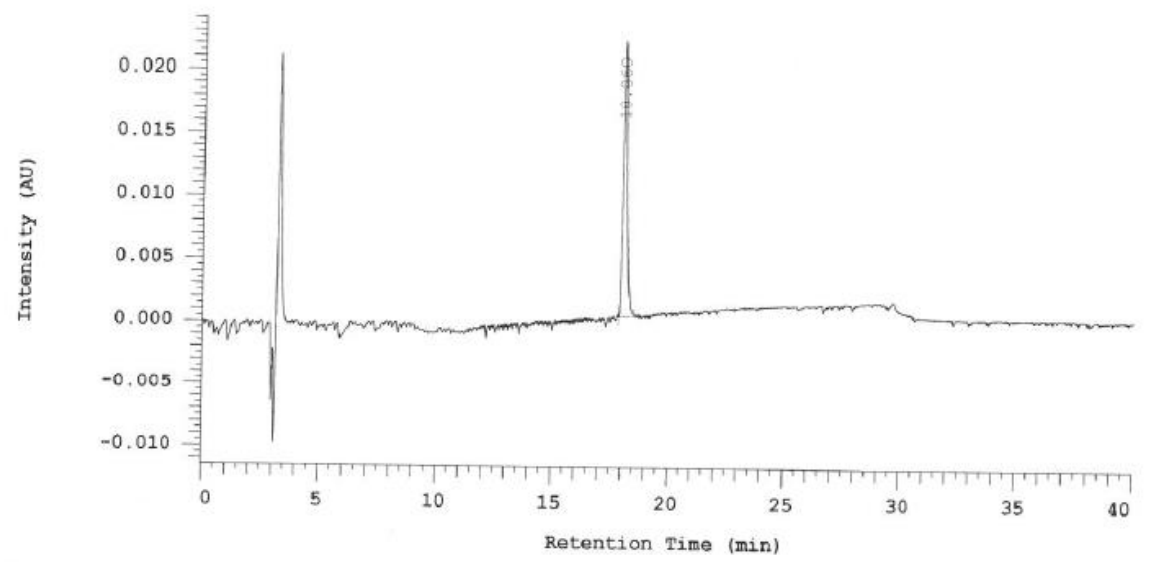

Pump 1: 5160

Pump 1 Solvent A: MeOH

Pump 1 Solvent C: $\mathrm{MeOH}$

Pump 1 Solvent B: Wasser

Pump 1 Solvent D: $\mathrm{MeOH}$

\begin{tabular}{ccccr} 
No. & RT & Area & Area \& & Height \\
\hline 1 & 18.060 & 127754 & 100.000 & 10869 \\
\hline & 127754 & 100.000 & 10869 \\
\hline
\end{tabular}


<smiles>COC(C)(C)c1ccc(C(=O)NCc2ccc(NS(C)(=O)=O)cc2Cl)cc1</smiles>

\section{$254 \mathrm{~nm}$}

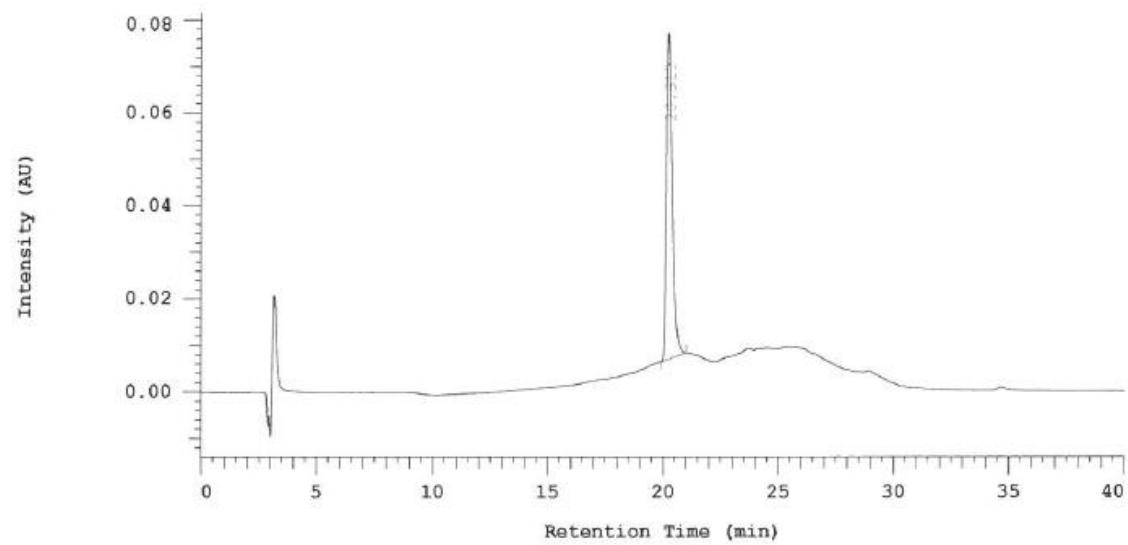

Pump 1: 5160

Pump 1 Solvent $A$ : MeOH

Pump 1 Solvent C: $\mathrm{MeOH}$

Pump 1 Solvent B: Wasser

Pump 1 Solvent D: $\mathrm{MeOH}$

\begin{tabular}{ccccr} 
No. & RT & Area & Area \% & Height \\
\hline 1 & 20.313 & 584444 & 100.000 & 24192 \\
\hline & & 584444 & 100.000 & 24192
\end{tabular}

\section{$280 \mathrm{~nm}$}

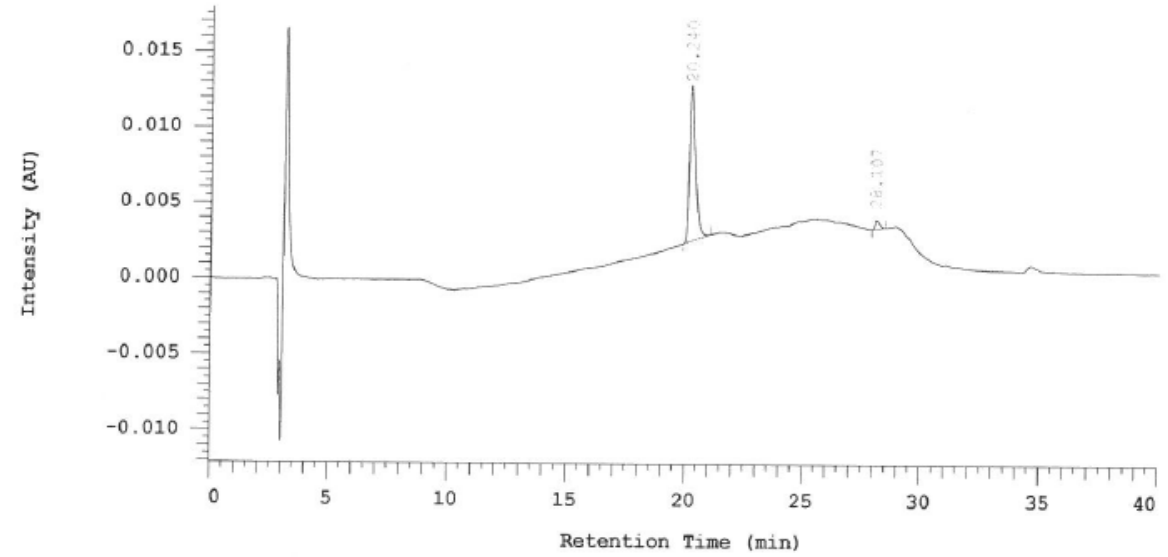

Pump 1: 5160

Pump 1 Solvent A: $\mathrm{MeOH}$

Pump 1 Solvent $\mathrm{C}$ : $\mathrm{MeOH}$

Pump 1 Solvent B: Wasser

Pump 1 Solvent D: $\mathrm{MeOH}$

\begin{tabular}{rrrrr} 
No. & RT & Area & Area \& & Height \\
\hline 1 & 20.240 & 86086 & 95.596 & 4903 \\
2 & 28.107 & 3966 & 4.404 & 285 \\
\hline & & 90052 & 100.000 & 5188
\end{tabular}


4-(3-hydroxyoxetan-3-yl)- $N$-(2-chloro-4-(methylsulfonamido)benzyl)benzamide (25)<smiles>CS(=O)(=O)Nc1ccc(CNC(=O)c2ccc(C3(O)COC3)cc2)c(Cl)c1</smiles>

\section{$254 \mathrm{~nm}$}

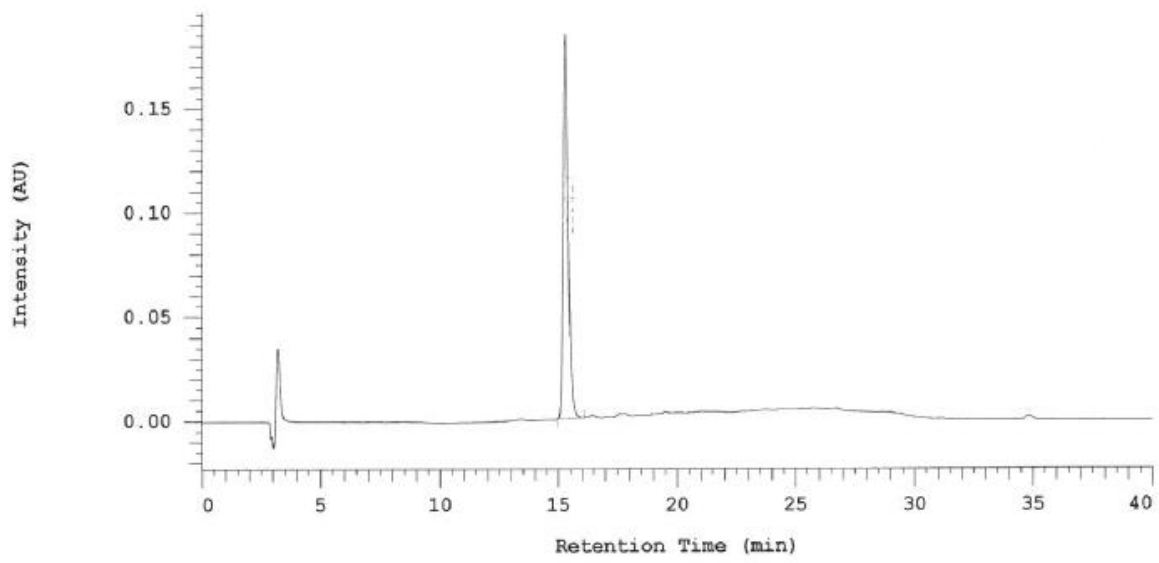

Pump 1: 5160

Pump 1 Solvent A: MeOH Pump 1 Solvent B: Wasser

Pump 1 Solvent C: $\mathrm{MeOH}$

Pump 1 Solvent D: $\mathrm{MeOH}$

\begin{tabular}{ccccr} 
No. & RT & Area & Area o & Height \\
\hline 1 & 15.427 & 1363799 & 100.000 & 41143 \\
\hline & & 1363799 & 100.000 & 41143
\end{tabular}

\section{$280 \mathrm{~nm}$}

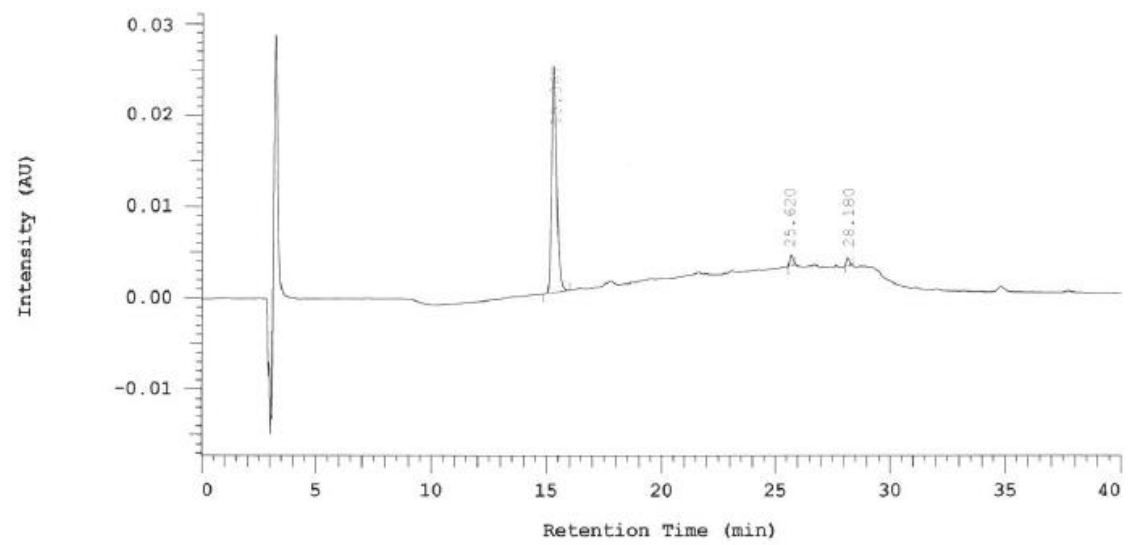

Pump 1: 5160

Pump 1 Solvent A: $\mathrm{MeOH}$

Pump 1 Solvent C: $\mathrm{MeOH}$

Pump 1 Solvent B: Wasser

Pump 1 Solvent D: MeOH

\begin{tabular}{rrrrr} 
No. & \multicolumn{1}{c}{ RT } & Area & Area \& & Height \\
\hline 1 & 15.367 & 182747 & 95.038 & 8521 \\
2 & 25.620 & 5531 & 2.876 & 385 \\
3 & 28.180 & 4010 & 2.086 & 363 \\
\hline & & 192288 & 100.000 & 9269
\end{tabular}


4-(1,1-Difluoroethyl)-N-(2-chloro-4-(cyclopropylsulfonamido)benzyl)benzamide (26)<smiles>CS(=O)(=O)Nc1ccc(CNC(=O)c2ccc(C(F)(F)F)cc2)c(Cl)c1</smiles>

\section{$254 \mathrm{~nm}$}

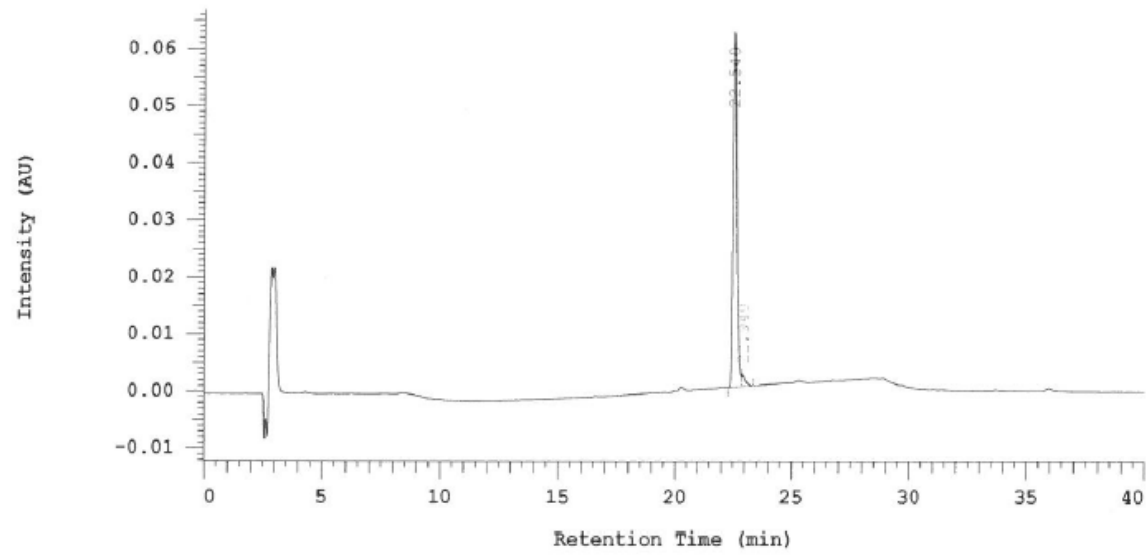

Pump 1: 5160

Pump 1 Solvent A: MeOH Pump 1 Solvent B: Wasser

Pump 1 Solvent C: $\mathrm{MeOH}$ Pump 1 Solvent D: MeOH

\begin{tabular}{rrrrr} 
No. & RT & Area & Area \% & Height \\
\hline 1 & 22.540 & 324550 & 96.761 & 31112 \\
2 & 22.940 & 10864 & 3.239 & 946 \\
\hline & & 335414 & 100.000 & 32058
\end{tabular}

\section{$280 \mathrm{~nm}$}

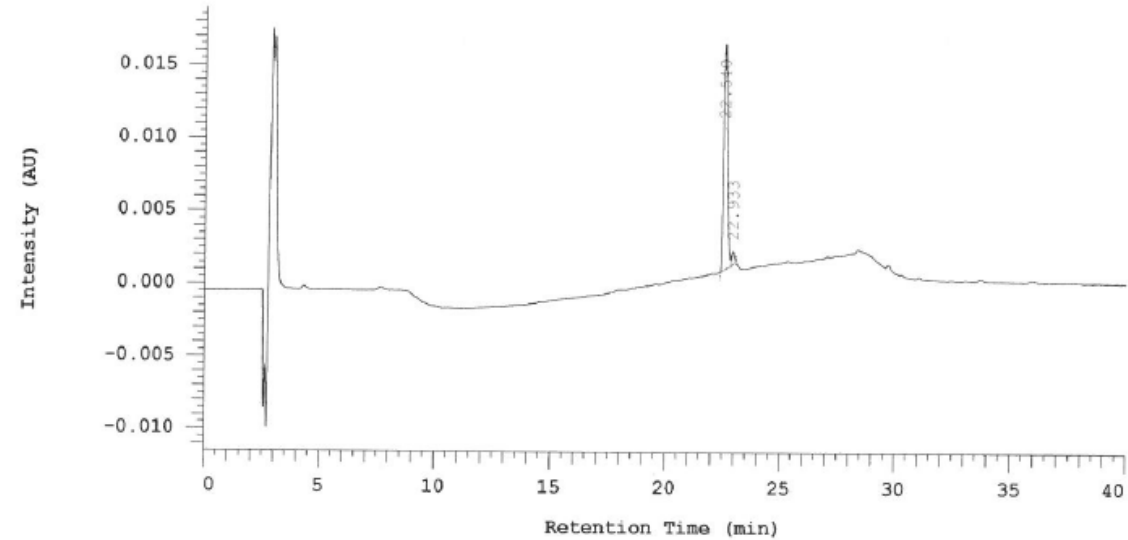

Pump 1: 5160

Pump 1 Solvent A: MeOH

Pump 1 Solvent C: $\mathrm{MeOH}$

Pump 1 Solvent B: Wasser

Pump 1 Solvent D: $\mathrm{MeOH}$

\begin{tabular}{rrrrr} 
No. & RT & Area & Area \& & Height \\
\hline 1 & 22.540 & 79296 & 95.340 & 7764 \\
2 & 22.933 & 3876 & 4.660 & 449 \\
\hline & & 83172 & 100.000 & 8213
\end{tabular}


<smiles>CS(=O)(=O)Nc1ccc(CNC(=O)c2ccc(CC(F)(F)F)cc2)c(Cl)c1</smiles>

\section{$254 \mathrm{~nm}$}

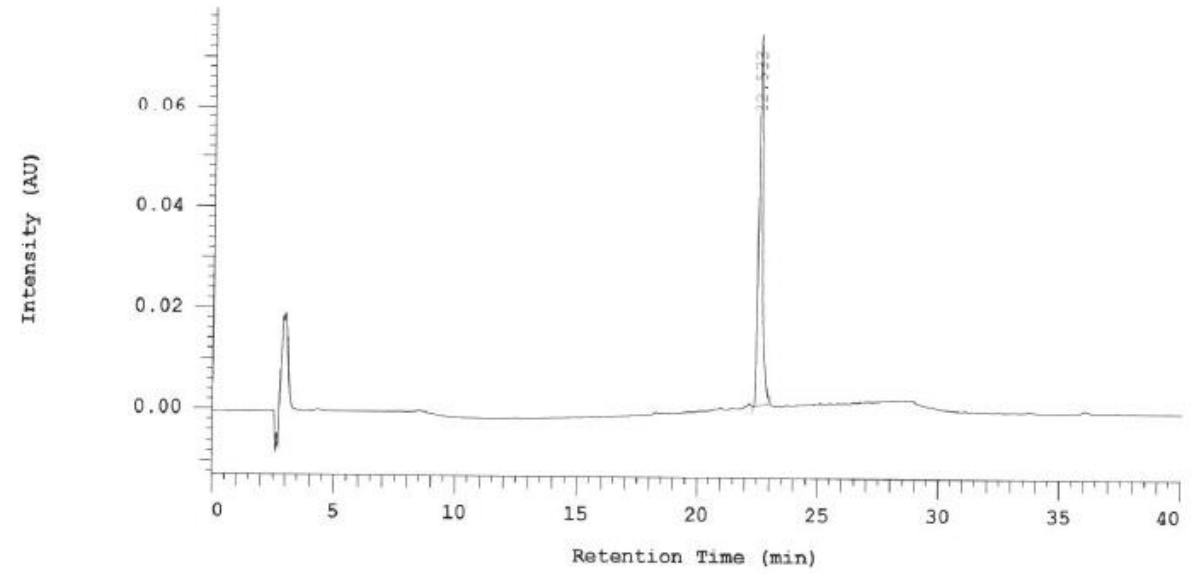

Pump 1: 5160

Pump 1 Solvent A: $\mathrm{MeOH}$

Pump 1 Solvent $\mathrm{C}$ : $\mathrm{MeOH}$

Pump 1 Solvent B: Wasser

Pump 1 Solvent D: $\mathrm{MeOH}$

\begin{tabular}{ccccr} 
No. & RT & Area & Area $\%$ & Height \\
\hline 1 & 22.533 & 398051 & 100.000 & 36865 \\
\hline & & 398051 & 100.000 & 36865 \\
\hline
\end{tabular}

\section{$280 \mathrm{~nm}$}

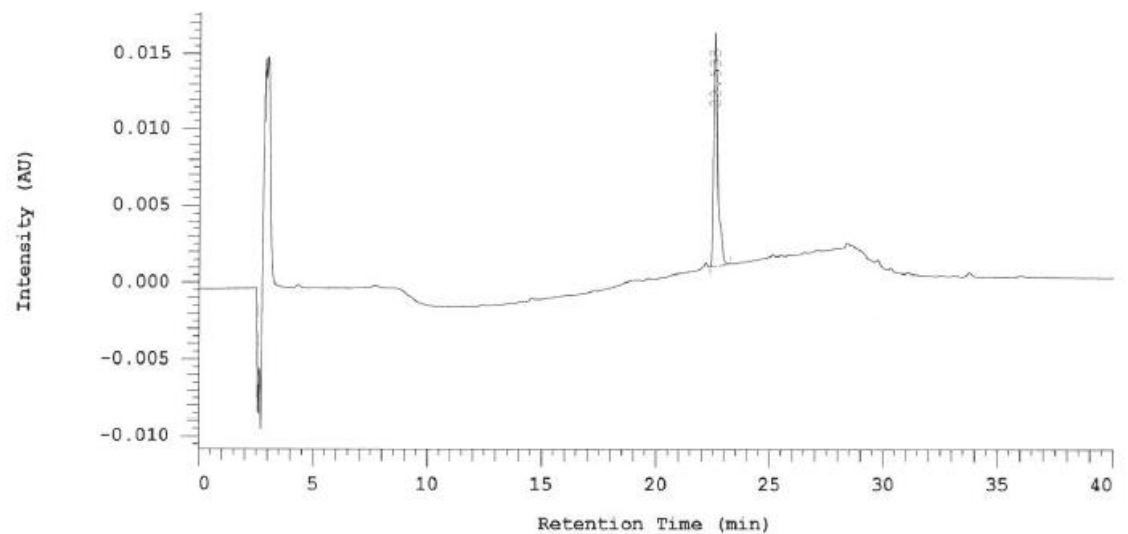

Pump 1: 5160

Pump 1 Solvent A: MeOH

Pump 1 Solvent C: $\mathrm{MeOH}$

Pump 1 Solvent B: Wasser

Pump 1 Solvent D: $\mathrm{MeOH}$

\begin{tabular}{rrrrr} 
No. & RT & Area & Area \& & Height \\
\hline 1 & 22.533 & 89313 & 100.000 & 7608 \\
\hline & & 89313 & 100.000 & 7608
\end{tabular}


4-(Pentafluoroethyl)-N-(2-fluoro-4-(methylsulfonamido)benzyl)benzamide (28)<smiles>CS(=O)(=O)Nc1ccc(CNC(=O)c2ccc(C(F)(F)F)cc2)c(Cl)c1</smiles>

$254 \mathrm{~nm}$

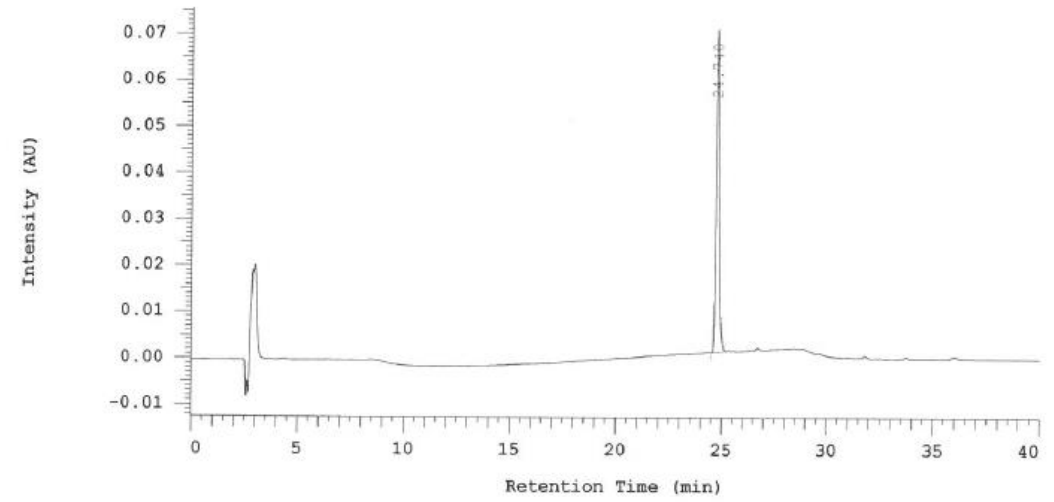

Pump 1: 5160

$\begin{array}{ll}\text { Pump } 1 \text { Solvent A: MeOH } & \text { Pump } 1 \text { Solvent B: Wasser } \\ \text { Pump I Solvent C: MeOH } & \text { Pump } 1 \text { Solvent D: MeOH }\end{array}$

Pump I Solvent C: MeOH

\begin{tabular}{ccccr} 
No. & RT & Area & Area & Height \\
\hline 1 & 24.740 & 346730 & 100.000 & 34869 \\
\hline & & 346730 & 100.000 & 34869
\end{tabular}

$280 \mathrm{~nm}$

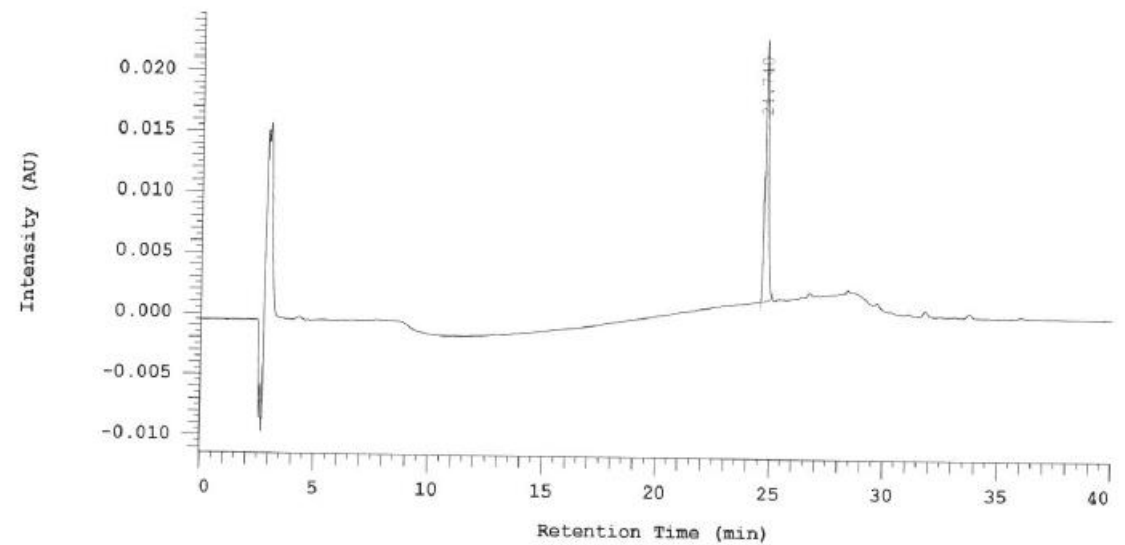

Pump 1: 5160

Pump 1 Solvent A: $\mathrm{MeOH}$

Pump 1 Solvent C: $\mathrm{MeOH}$

Pump 1 Solvent B: Wasser

Pump 1 Solvent D: $\mathrm{MeOH}$

\begin{tabular}{ccccc} 
No. & RT & Area & Area 8 & Height \\
\hline 1 & 24.740 & 105740 & 100.000 & 10679 \\
\hline & 105740 & 100.000 & 10679
\end{tabular}


<smiles>CS(=O)(=O)Nc1ccc(CNC(=O)c2ccc(C(F)(F)F)cc2)c(Cl)c1</smiles>

\section{$254 \mathrm{~nm}$}

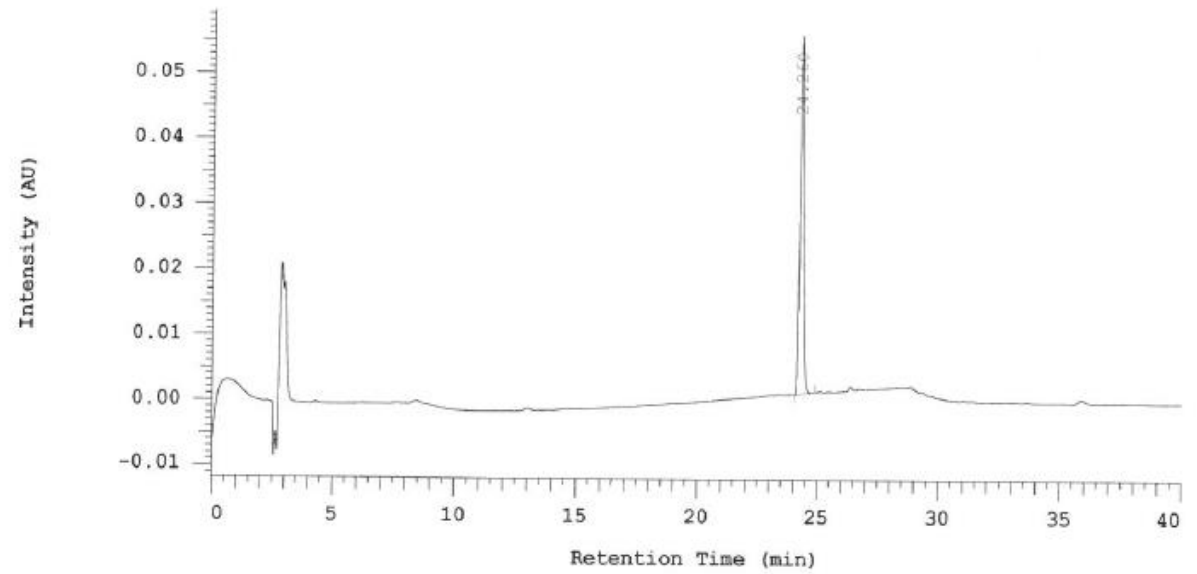

Pump 1: 5160

Pump 1 Solvent A: $\mathrm{MeOH}$

Pump 1 Solvent C: MeOH

Pump 1 Solvent B: Wasser

\begin{tabular}{ccccc} 
No. & RT & Area & Area \& & Height \\
\hline 1 & 24.260 & 276269 & 100.000 & 27400 \\
\hline & 276269 & 100.000 & 27400
\end{tabular}

$280 \mathrm{~nm}$

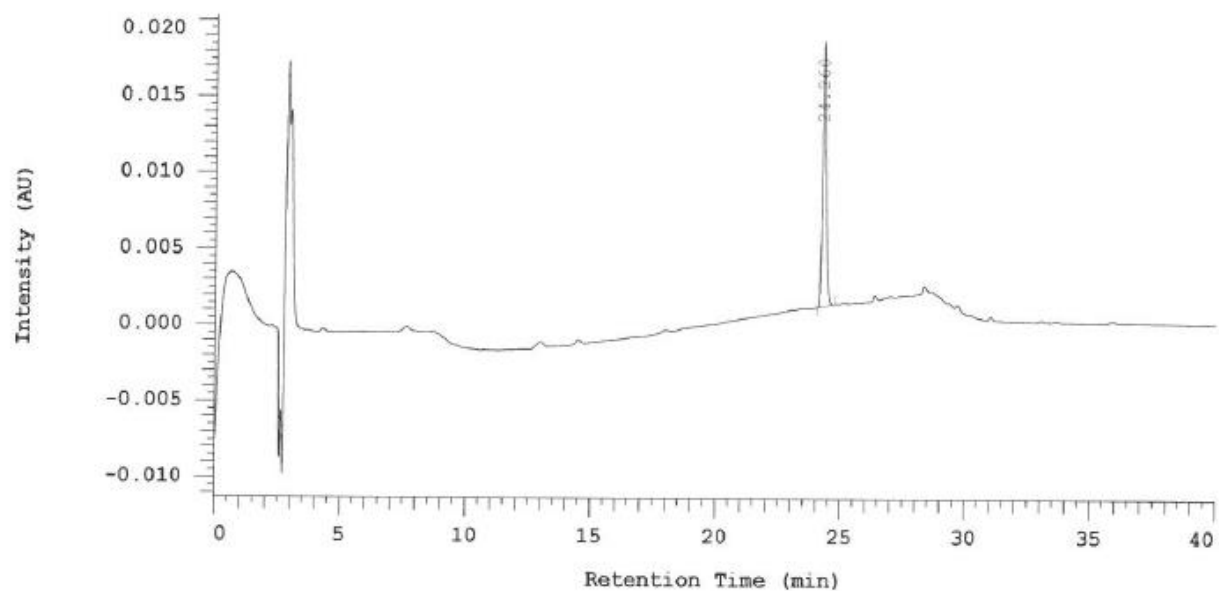

Pump 1: 5160

Pump 1 Solvent A: $\mathrm{MeOH}$

Pump 1 Solvent C: $\mathrm{MeOH}$

Pump 1 Solvent B: Wasser

Pump 1 Solvent D: $\mathrm{MeOH}$

\begin{tabular}{rrrrr} 
No. & RT & Area & Area \% & Height \\
\hline 1 & 24.260 & 87702 & 100.000 & 8702 \\
\hline & 87702 & 100.000 & 8702
\end{tabular}

